\title{
LOCAL EXACT CONTROLLABILITY TO THE TRAJECTORIES OF THE NAVIER-STOKES SYSTEM WITH NONLINEAR NAVIER-SLIP BOUNDARY CONDITIONS*
}

\author{
Sergio Guerrero ${ }^{1}$
}

\begin{abstract}
In this paper we deal with the local exact controllability of the Navier-Stokes system with nonlinear Navier-slip boundary conditions and distributed controls supported in small sets. In a first step, we prove a Carleman inequality for the linearized Navier-Stokes system, which leads to null controllability of this system at any time $T>0$. Then, fixed point arguments lead to the deduction of a local result concerning the exact controllability to the trajectories of the Navier-Stokes system.
\end{abstract}

Mathematics Subject Classification. 34B15, 35Q30, 76D03, 93B05, 93C10.

Received February 9, 2004. Revised April 19, 2005.

\section{INTRODUCTION}

Let $\Omega \subset \mathbf{R}^{N}$ ( $N=2$ or 3 ) be a bounded connected open set whose boundary $\partial \Omega$ is regular enough (for instance, $\partial \Omega \in C^{2}$ ). Let $\omega \subset \Omega$ be a (small) nonempty open subset and let $T>0$. We will use the notation $Q=\Omega \times(0, T)$ and $\Sigma=\partial \Omega \times(0, T)$ and we will denote by $n(x)$ the outward unit normal to $\Omega$ at the point $x \in \partial \Omega$.

On the other hand, we will denote by $C$ a generic positive constant (usually depending on $\Omega$ and $\omega$ ).

Let us consider the controlled Navier-Stokes system with nonlinear Navier slip boundary conditions. Given a nonlinear 'regular' function $f: \mathbf{R}^{N} \mapsto \mathbf{R}^{N}$ and an initial state $y^{0}$, we consider the following system:

$$
\begin{cases}y_{t}-\nabla \cdot(D y)+(y, \nabla) y+\nabla p=v 1_{\omega} & \text { in } Q, \\ \nabla \cdot y=0 & \text { in } Q, \\ y \cdot n=0, \quad(\sigma(y, p) \cdot n)_{t g}+f(y)_{t g}=0 & \text { on } \Sigma, \\ y(\cdot, 0)=y^{0}(\cdot) & \text { in } \Omega,\end{cases}
$$

where

is the stress tensor and

$$
\sigma(y, p)=-p I d+D y
$$

$$
(D y)_{i, j}=\partial_{j} y^{i}+\partial_{i} y^{j} \quad(1 \leq i, j \leq N)
$$

Keywords and phrases. Navier-Stokes system, controllability, slip.

* This work has been partially supported by D.G.E.S. (Spain), Grant BFM2000-1317.

${ }^{1}$ Dpto. E.D.A.N., University of Sevilla, Aptdo. 1160, 41080 Sevilla, Spain; sguerrero@us.es. 
is the deformation tensor. Here $v$ denotes the control function which acts over system (1) trough $\omega$ during the time $T$. On the other hand, the subscript "tg" stands for the tangential component of the corresponding vector field, i.e.:

$$
w_{t g}=w-(w \cdot n) n
$$

The first of the boundary conditions is the slip condition and says that the particles in the fluid do not penetrate the boundary. On the other hand and in order to explain the second one, let us remark that $\sigma(y, p) \cdot n$ is the force exerted by the fluid over the solid wall. Thus, a condition like

$$
(\sigma(y, p) \cdot n)_{t g}=-k y_{t g} \quad k \text { constant }
$$

mean that the tangential component of this force is proportional to the velocity field on the boundary. But $k$ may not depend on $|y|$ linearly, so

$$
(\sigma(y, p) \cdot n)_{t g}=-(f(y))_{t g}
$$

stands for a more general condition. Let us also point out that this kind of conditions appear in turbulence. In such a context, we cannot expect to exactly find neither $y$ nor $p$, not even approximatively. Then, one is led to "average" the Navier-Stokes equations and solve them. When solid walls are concerned, experimental analysis show that these averaged variables behave like

$$
y \cdot n=0 \quad \text { and } \quad(\sigma(y, p) \cdot n)_{t g}=-f(y)_{t g}
$$

near the boundary. This is known as "wall law". For further literature on this subject, see for instance $[5,18,19]$.

In the context of controllability, this problem has not been studied up to now, so their controllability properties are somehow unknown.

A related linear control system which will be useful in this paper is

$$
\begin{cases}w_{t}-\nabla \cdot(D w)+(a(x, t)+b(x, t), \nabla) w+(w, \nabla) b(x, t)+\nabla q=v 1_{\omega} & \text { in } Q, \\ \nabla \cdot w=0 & \text { in } Q, \\ w \cdot n=0, \quad(\sigma(w, q) \cdot n)_{t g}+(A(x, t) w)_{t g}=0 & \text { on } \Sigma, \\ w(\cdot, 0)=w^{0}(\cdot) & \text { in } \Omega,\end{cases}
$$

with $A \in L^{\infty}\left(\mathbf{R}^{N} ; \mathbf{R}^{N}\right)$ a $N \times N$ matrix function and $a$ and $b$ divergence free vector field functions. Problems of this kind have already been studied in [6], where the author proved an approximate controllability result for system (2) in dimension 2 with $a \equiv b \equiv A \equiv 0$. Another result concerning the local exact controllability of the Navier-Stokes system with Navier slip boundary conditions was studied in [12].

Let us define the concepts of controllability which will be concerned in this paper. For system (2), we will say that it is null controllable if for any (suitable) $y^{0}$ there exists a control $v$ such that the associated solution to $(2)$ verifies

$$
w(\cdot, T)=0 \text { in } \Omega .
$$

For system (1), we will say that it is locally exactly controllable to the trajectories if for a suitable trajectory $(\bar{y}, \bar{p})$ of system (1) with no control, there exists $\delta>0$ such that

$$
\left\|y^{0}-\bar{y}(0)\right\|_{E} \leq \delta \Rightarrow \exists v: y(\cdot, T)=\bar{y}(\cdot, T) \text { in } \Omega,
$$

for some Banach space $E$. In fact, $(\bar{y}, \bar{p})$ will satisfy

$$
\begin{cases}\bar{y}_{t}-\Delta \bar{y}+(\bar{y}, \nabla) \bar{y}+\nabla \bar{p}=0 & \text { in } Q, \\ \nabla \cdot \bar{y}=0 & \text { in } Q, \\ \bar{y} \cdot n=0,(\sigma(\bar{y}, \bar{p}) \cdot n)_{t g}+(f(\bar{y}))_{t g}=0 & \text { on } \Sigma .\end{cases}
$$


The strategy of this paper will be, in a first step, to establish a null controllability result for (2) (see Th. 0.1), which can actually be seen as a linearization of system (1) around appropriate trajectories $\bar{y}$ of (1). Then, using this result and a fixed point argument, the local exact controllability to "regular enough" trajectories of system (1) will be deduced (see Th. 0.2).

Let us introduce several spaces which are usual in the context of problems modelling incompressible fluids:

$$
H=\left\{y \in L^{2}(\Omega)^{N}: \nabla \cdot y=0 \text { in } \Omega, y \cdot n=0 \text { on } \partial \Omega\right\}
$$

and

$$
W=\left\{y \in H^{1}(\Omega)^{N}: \nabla \cdot y=0 \text { in } \Omega, y \cdot n=0 \text { on } \partial \Omega\right\}
$$

In the sequel, some regularity assumptions will be imposed on the previous potentials and matrix functions. Let $0<\ell<1 / 2$ arbitrarily close to $1 / 2$. For vector fields $d(x, t)$, we will assume certain regularity hypothesis:

$$
d \in L^{\infty}(Q)^{N}, \quad d_{t} \in L^{2}\left(0, T ; L^{r}(\Omega)^{N}\right) \quad\left(\begin{array}{cc}
r=6 & \text { if } N=3 \\
r=4 & \text { if } N=2
\end{array}\right)
$$

while for a matrix function $A$ the following will be imposed:

$$
\begin{gathered}
A \in L^{\infty}(\Sigma)^{N \times N}, \\
A \in H^{1-\ell}\left(0, T ; W^{\nu_{1}, \nu_{1}+1}(\partial \Omega)^{N \times N}\right), \\
A \in H^{(3-\ell) / 2}\left(0, T ; H^{\nu_{2}}(\partial \Omega)^{N \times N}\right),
\end{gathered}
$$

where $\nu_{1}>1$ (arbitrarily small) in dimension 3 and $\nu_{1}=1$ in dimension 2 and $\nu_{2}=(1 / 2)(3-N)+(1-\ell)(N-2)$. We recall here the definition of the Sobolev spaces $W^{r_{1}, r_{2}}(\Omega)$ : for $r_{1} \in \mathbf{N}$, we have

$$
W^{r_{1}, r_{2}}(\Omega)=\left\{u \in L^{r_{2}}(\Omega): D^{\alpha} u \in L^{r_{2}}(\Omega), \alpha \in \mathbf{N}^{N},|\alpha| \leq r_{1}\right\}
$$

In general, for $r_{1} \in \mathbf{R}$, we define (see, for instance, $[1,16,17]$ )

$$
W^{r_{1}, r_{2}}(\Omega)=\left\{u \in W^{\left[r_{1}\right], r_{2}}(\Omega): \frac{\left|D^{\alpha} u(x)-D^{\alpha} u(y)\right|}{|x-y|^{r_{1}-\left[r_{1}\right]+N / r_{2}}} \in L^{r_{2}}(\Omega \times \Omega),|\alpha|=\left[r_{1}\right]\right\} .
$$

Another way to define $W^{r_{1}, r_{2}}(\Omega)$ is as an interpolation space between $W^{\left[r_{1}\right], r_{2}}(\Omega)$ and $W^{\left[r_{1}\right]+1, r_{2}}(\Omega)$.

In order to make these hypothesis more comprehensible, observe that for instance a function $A \in C^{3 / 2}(\bar{\Sigma})^{N \times N}$ would fulfill the previous properties.

As announced, the first main result of this paper concerns the null controllability of system (2) and is presented in the following theorem.

Theorem 0.1. Let $w^{0} \in H$ and let us suppose that $A$ verifies (8)-(10) and $a, b$ are divergence free vector fields verifying (7). Then, there exist controls $v \in H^{1}\left(0, T ; L^{2}(\omega)^{N}\right) \cap C^{0}\left([0, T] ; H^{1}(\omega)^{N}\right)$ such that the corresponding solution to (2) verifies (3).

Furthermore, there exists a positive constant $C$ depending on $\Omega, \omega, T,\|a\|_{\infty},\|b\|_{\infty},\left\|a_{t}\right\|_{L^{2}\left(L^{r}\right)},\left\|b_{t}\right\|_{L^{2}\left(L^{r}\right)}$, $\|A\|_{H^{1-\ell}\left(W^{\nu_{1}, \nu_{1}+1}\right)}$ and $\|A\|_{H^{(3-\ell) / 2}\left(H^{\nu_{2}}\right)}$, such that

$$
\|v\|_{H^{1}\left(L^{2}\right)}+\|v\|_{L^{\infty}\left(H^{1}\right)} \leq C\left\|w^{0}\right\|_{H} .
$$


The proof of Theorem 0.1 is based on the obtention of the so-called observability inequality for a backwards system associated to (2). In fact, we will consider the adjoint system

$$
\begin{cases}-\varphi_{t}-\nabla \cdot(D \varphi)-(a(x, t), \nabla) \varphi-D \varphi b(x, t)+\nabla \pi=0 & \text { in } Q \\ \nabla \cdot \varphi=0 & \text { in } Q \\ \varphi \cdot n=0, \quad(\sigma(\varphi, \pi) \cdot n)_{t g}+\left(A(x, t)^{t} \varphi\right)_{t g}=0 & \text { on } \Sigma \\ \varphi(\cdot, T)=\varphi^{0}(\cdot) & \text { in } \Omega .\end{cases}
$$

The usual tools to obtain the observability for (11) are global Carleman inequalities. This was popularized by Imanuvilov and Fursikov in [10] and several advances have been made since then (see, for instance, [13-15]). The proof of the corresponding Carleman inequality for this system will be divided in two steps:

- We first obtain a Carleman inequality for a heat system associated to (11). Precisely, we consider a function $\varphi$ verifying

$$
\begin{cases}-\varphi_{t}-\nabla \cdot(D \varphi)=G \in L^{2}(Q)^{N}, \quad \nabla \cdot \varphi=0 & \text { in } Q \\ \varphi \cdot n=0, \quad(D \varphi \cdot n)_{t g}+(A(x, t) \varphi)_{t g}=0 & \text { on } \Sigma .\end{cases}
$$

Similar techniques to those developed in [10] are employed in order to get the desired estimate. More details will be given in Section 3.1.

- Then, following the general ideas of $[8,13]$, a Carleman inequality for system (11) is established. Let us remark here the particular difficulty an estimate of this kind contains due to the coupling boundary conditions. The details are given in Section 3.2.

This usually provides $L^{2}$ controls leading to the null controllability of the velocity vector field solution of (2). However, in order to perform a fixed point argument and extract some controllability properties for the nonlinear system (1), a more regular control is needed. The regularization process we use here was introduced in [3].

The second main result of this paper concerns the local exact controllability to the trajectories of (1). Several regularity hypotheses have to be assumed for the trajectories in order to be able to approach them:

$$
\begin{gathered}
\bar{y} \in L^{\infty}(Q)^{N}, \quad \bar{y} \in H^{1-\ell}\left(0, T ; W^{\nu_{1}, \nu_{1}+1}(\partial \Omega)^{N}\right), \\
\bar{y} \in H^{(3-\ell) / 2}\left(0, T ; H^{\nu_{2}}(\partial \Omega)^{N}\right), \\
\bar{y}(\cdot, 0) \in H^{3}(\Omega)^{N} \cap W, \\
(D \bar{y}(\cdot, 0) \cdot n)_{t g}+(f(\bar{y}(\cdot, 0)))_{t g}=0 \text { on } \partial \Omega .
\end{gathered}
$$

Observe that, with a suitable initial condition, $\bar{y} \in C^{3 / 2}(\bar{\Sigma})^{N}$ would also suffice here to assure the the above properties. On the other hand, we will impose regularity to the nonlinearities appearing on the boundary condition:

$$
f \in C^{3}\left(\mathbf{R}^{N} ; \mathbf{R}^{N}\right) .
$$

Theorem 0.2. Let $f$ verify (14), and let $y^{0} \in H^{3}(\Omega)^{N} \cap W$ satisfy the compatibility condition

$$
\left(D y^{0} \cdot n\right)_{t g}+\left(f\left(y^{0}\right)\right)_{t g}=0 \text { on } \partial \Omega
$$

Then, the exact controllability to the trajectories of (1) satisfying (12)-(13) holds, i.e., there exists $\delta>0$ such that if $\left\|y^{0}-\bar{y}(\cdot, 0)\right\|_{H^{3} \cap W} \leq \delta$, we can find controls $v$ such that the corresponding solutions $y$ to (1) satisfy

$$
y(\cdot, T)=\bar{y}(\cdot, T) \text { in } \Omega .
$$

Furthermore, these controls belong to

$$
H^{1}\left(0, T ; L^{2}(\omega)^{N}\right) \cap C\left([0, T] ; H^{1}(\omega)^{N}\right) .
$$


A fixed point technique is used to prove this result. This tool has successfully been used in this context several times; see, for instance, $[7,9,23]$. We apply here Kakutani's theorem.

The main difficulty arising in this situation turns to be the restrictive spaces one is forced to deal with when proving compactness results for linear systems like (2). There, the regularity results which will be proved in Section 2, are crucial.

In spite of this positive controllability result for system (1), this is still far from what would be desirable for systems of this kind. It would be interesting to know whether one has local exact controllability to all bounded trajectories. However, this seems to be a very complicated question.

The paper is organized as follows: in Section 2 some previous and technical regularity results for systems of this kind are established. Section 3 will contain the proofs of the Carleman inequalities. Finally, the controllability results are proved in Section 4 (Ths. 1 and 2).

\section{Preliminary Results}

In this section, we will prove several technical results which will be used later on. More precisely, we present two regularity results concerning the Stokes system with linear Navier-slip boundary conditions.

The first one concerns the existence of strong solutions, i.e., solutions $(u, \theta)$ belonging to the space

$$
\left(L^{2}\left(0, T ; H^{2}(\Omega)^{N} \cap W\right) \cap H^{1}(0, T ; H)\right) \times L^{2}\left(0, T ; H^{1}(\Omega)\right) .
$$

We give it in the following proposition:

Proposition 1.1. Let A verify (8), $u^{0} \in H, f_{1} \in L^{2}\left(0, T ; W^{\prime}\right), f_{2} \in L^{2}\left(0, T ; H^{-1 / 2}(\partial \Omega)^{N}\right)$ and let $u$ be the weak solution of the system

$$
\begin{cases}u_{t}-\nabla \cdot(D u)+\nabla \theta=f_{1} & \text { in } Q, \\ \nabla \cdot u=0 & \text { in } Q, \\ u \cdot n=0, \quad(\sigma(u, \theta))_{t g}+(A(x, t) u)_{t g}=f_{2} & \text { on } \Sigma, \\ u(\cdot, 0)=u^{0}(\cdot) & \text { in } \Omega,\end{cases}
$$

namely, the function u satisfying

$$
\left\{\begin{array}{l}
\int_{\Omega} u_{t}(t) \cdot v \mathrm{~d} x+\frac{1}{2} \int_{\Omega} D u(t): D v \mathrm{~d} x+\int_{\partial \Omega} A u(t) \cdot v \mathrm{~d} \sigma \\
=\int_{\Omega} f_{1}(t) \cdot v \mathrm{~d} x+\int_{\partial \Omega} f_{2}(t) \cdot v \mathrm{~d} \sigma \quad \text { a.e. } t \in(0, T) \quad \forall v \in W, \\
u(\cdot, 0)=u^{0}(\cdot) \text { in } \Omega .
\end{array}\right.
$$

Then, if we also suppose that $A$ verifies (9), $u^{0} \in W$ and

$$
f_{1} \in L^{2}(Q)^{N}, f_{2} \in L^{2}\left(0, T ; H^{1 / 2}(\partial \Omega)^{N}\right), f_{2} \in H^{(1-\ell) / 2}\left(0, T ; H^{\ell-1 / 2}(\partial \Omega)^{N}\right),
$$

$u$ is actually, together with a pressure $\theta$, the strong solution of (16), i.e.,

$$
\begin{gathered}
u \in L^{2}\left(0, T ; H^{2}(\Omega)^{N} \cap W\right), \quad u_{t} \in L^{2}(0, T ; H), \quad u \in L^{\infty}(0, T ; W), \\
\theta \in L^{2}\left(0, T ; H^{1}(\Omega)\right) .
\end{gathered}
$$


Furthermore, there exists a positive constant $C$ such that

$$
\begin{aligned}
&\left\|u_{t}\right\|_{L^{2}(Q)^{N}}^{2}+\|u\|_{L^{2}\left(H^{2} \cap W\right)}^{2}+\|u\|_{L^{\infty}\left(H^{1}\right)}^{2}+\|\theta\|_{L^{2}\left(H^{1}\right)}^{2} \\
& \leq C \mathrm{e}^{C T\|A\|_{P}^{2}\left(1+\|A\|_{P}^{4}\right)\left(\left\|f_{1}\right\|_{L^{2}(Q)^{N}}^{2}+\left\|f_{2}\right\|_{L^{2}\left(H^{1 / 2}\right)}^{2}\right.} \\
&\left.+\left\|f_{2}\right\|_{H^{(1-\ell) / 2}\left(H^{\ell-1 / 2}\right)}^{2}+\left\|u^{0}\right\|_{H^{1}(\Omega)^{N}}^{2}\right)
\end{aligned}
$$

where the space $P$ is given by

$$
P=H^{1-\ell}\left(0, T ; W^{\nu_{1}, \nu_{1}+1}(\partial \Omega)^{N \times N}\right) .
$$

Proof. We remember here that the definitions of $H$ and $W$ were given in (5) and (6) at the beginning of the paper. A classical Galerkin's method can be employed in order to prove the existence and uniqueness and obtain estimates of $u$ as weak solution of (16). In fact, multiplying the equation in (16) by $u(t)$ and integrating in $\Omega$, we obtain

$$
\begin{aligned}
& \frac{1}{2} \frac{\mathrm{d}}{\mathrm{d} t} \int_{\Omega}|u(t)|^{2} \mathrm{~d} x+\frac{1}{2} \int_{\Omega}|D u(t)|^{2} \mathrm{~d} x+\int_{\partial \Omega}(A u)(t) \cdot u(t) \mathrm{d} \sigma \\
& =\left\langle f_{1}(t), u(t)\right\rangle_{W^{\prime}, W}+\left\langle f_{2}(t), u(t)\right\rangle_{\partial \Omega}
\end{aligned}
$$

for a.e. $t \in(0, T)$, where we have denoted $\langle\cdot, \cdot\rangle$ to the duality product between $H^{-1 / 2}(\partial \Omega)$ and $H^{1 / 2}(\partial \Omega)$. From the fact that $\|D u\|_{L^{2}(\Omega)^{N}}$ is a norm in $W$ equivalent to that of $H^{1}(\Omega)^{N}$ (Korn's inequality; see, for instance, [20]) and the trace inequality

$$
\int_{\partial \Omega}|u(t)|^{2} \mathrm{~d} \sigma \leq C\|u(t)\|_{L^{2}(\Omega)^{N}}\|u(t)\|_{H^{1}(\Omega)^{N}}
$$

we find

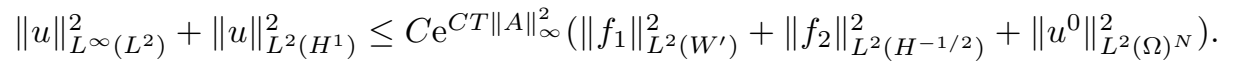

Let us now multiply the equation in (16) by $u_{t}$ and integrate in $\Omega$. This yields

$$
\begin{aligned}
\int_{\Omega}\left|u_{t}(t)\right|^{2} \mathrm{~d} x+\frac{1}{4} \frac{\mathrm{d}}{\mathrm{d} t} \int_{\Omega}|D u(t)|^{2} \mathrm{~d} x-\left\langle f_{2}(t), u_{t}(t)\right\rangle_{\partial \Omega} & \\
& =-\int_{\partial \Omega}(A u(t)) \cdot u_{t}(t) \mathrm{d} \sigma+\int_{\Omega} f_{1}(t) \cdot u_{t}(t) \mathrm{d} x \quad \text { a.e. } t \in(0, T) .
\end{aligned}
$$

Using $f_{1} \in L^{2}(Q)^{N}$, we have

$$
\begin{aligned}
\frac{1}{2} \int_{\Omega}\left|u_{t}(t)\right|^{2} \mathrm{~d} x+\frac{1}{4} \frac{\mathrm{d}}{\mathrm{d} t} \int_{\Omega}|D u(t)|^{2} \mathrm{~d} x-\left\langle f_{2}(t),\right. & \left.u_{t}(t)\right\rangle_{\partial \Omega} \\
& \leq \frac{1}{2} \int_{\Omega}\left|f_{1}(t)\right|^{2} \mathrm{~d} x-\int_{\partial \Omega}(A u(t)) \cdot u_{t}(t) \mathrm{d} \sigma \quad \text { a.e. } t \in(0, T) .
\end{aligned}
$$

Next, we see (16) like a stationary system, that is to say,

$$
\begin{cases}-\Delta u(t)+\nabla \theta(t)=f_{1}(t)-u_{t}(t) & \text { in } \Omega, \\ \nabla \cdot u(t)=0 & \text { in } \Omega, \\ u(t) \cdot n=0, \quad(\sigma(u(t), \theta(t)))_{t g}+(A(x, t) u(t))_{t g}=f_{2}(t) & \text { on } \partial \Omega,\end{cases}
$$


for almost every $t \in(0, T)$. The goal will be to prove that the weak solution $(u, \theta)$ of the system

$$
\begin{cases}-\Delta u+\nabla \theta=g & \text { in } \Omega, \\ \nabla \cdot u=0 & \text { in } \Omega, \\ u \cdot n=0, \quad(\sigma(u, \theta))_{t g}=f_{3} & \text { on } \partial \Omega,\end{cases}
$$

actually belongs to

$$
\left(H^{2}(\Omega)^{N} \cap W\right) \times H^{1}(\Omega)
$$

whenever $g \in L^{2}(\Omega)^{N}$ (see (22)) and suitable $f_{3}$. The proof we develop here follows the ideas of [4].

Let us first remark that the weak solution of $(24)$ verifies

$$
\|u\|_{H^{1}(\Omega)^{N}}+\|\theta\|_{L^{2}(\Omega)} \leq C\left(\|g\|_{W^{\prime}}+\left\|f_{3}\right\|_{H^{-1 / 2}(\partial \Omega)^{N}}\right)
$$

for a positive constant $C$.

The interior regularity readily follows from the corresponding result with Dirichlet conditions and that can be founded in [22], for instance. Then, for every $\Omega^{\prime} \subset \subset \Omega$, we have $u \in H^{2}\left(\Omega^{\prime}\right)^{N}, \theta \in H^{1}\left(\Omega^{\prime}\right)$ and

$$
\|u\|_{H^{2}\left(\Omega^{\prime}\right)^{N}}+\|\theta\|_{H^{1}\left(\Omega^{\prime}\right)} \leq C\|g\|_{L^{2}(\Omega)^{N}},
$$

for some positive constant $C\left(\Omega^{\prime}, \Omega\right)$.

Let $x^{0} \in \partial \Omega$ and let $U_{0}$ be a neighborhood of $x^{0}$. Then, we will prove that $u \in H^{2}(\Omega \cap \tilde{U})^{N}$ and $\theta \in H^{1}(\Omega \cap \tilde{U})$, for every $\tilde{U} \subset \subset U_{0}$. To this end, let $\psi$ be a $W^{2, \infty}$ diffeomorphism which sends the set

$$
C_{0}=\left\{\left(\xi^{\prime}, \xi_{N}\right) \in \mathbf{R}^{N}:\left|\xi_{i}\right|<\alpha_{0} \quad i=1, \ldots, N-1,\left|\xi_{N}\right|<\beta_{0}\right\}
$$

into and onto $U_{0}$ and which verifies $\psi\left(C_{0}^{+}\right)=\Omega \cap U_{0}$ and $\psi\left(\Delta_{\alpha_{0}}\right)=\partial \Omega \cap U_{0}$. Here, we have denoted $C_{0}^{+}=C_{0} \cap \mathbf{R}_{+}^{N}$ and $\Delta_{\alpha_{0}}=\partial \mathbf{R}_{+}^{N} \cap C_{0}$. Let us now introduce a cut-off function $\zeta \in C^{2}\left(U_{0}\right)$ such that

$$
\zeta \equiv 1 \text { in } \tilde{U} \text { and } \operatorname{supp} \zeta \subset U_{1} \subset \subset U_{0},
$$

where $U_{1}$ is a regular open set.

Then, let us set $z=\zeta u, h=\zeta \theta$. They verify:

$$
\begin{cases}-\nabla \cdot(D z)+\nabla h=g^{*} & \text { in } \Omega \cap U_{0}, \\ \nabla \cdot z=g_{1}^{*} & \text { in } \Omega \cap U_{0} \\ z \cdot n=0, \quad(\sigma(z, h) \cdot n)_{t g}=g_{2}^{*} & \text { on } \partial \Omega \cap U_{0} \\ z=0 & \text { on } \Omega \cap \partial U_{0}\end{cases}
$$

with

$$
\begin{gathered}
g^{*}=-\zeta g-2 \nabla \zeta \cdot \nabla u-\nabla \zeta \cdot \nabla^{t} u-\Delta \zeta u-\nabla \nabla \zeta \cdot u+\theta \nabla \zeta \in L^{2}\left(\Omega \cap U_{0}\right)^{N}, \\
g_{1}^{*}=\nabla \zeta \cdot u \in H^{1}\left(\Omega \cap U_{0}\right) \quad \text { and } \quad g_{2}^{*}=\zeta f_{3}+\frac{\partial \zeta}{\partial n} u \in H^{1 / 2}\left(\partial \Omega \cap U_{0}\right)^{N} .
\end{gathered}
$$

The weak solution of (27) is given by $(z, h) \in X \times L^{2}\left(\Omega \cap U_{0}\right)$ satisfying

$$
\left\{\begin{array}{c}
\int_{\Omega \cap U_{0}} D z: \nabla v \mathrm{~d} x-\int_{\Omega \cap U_{0}} h \nabla \cdot v \mathrm{~d} x \\
=\int_{\Omega \cap U_{0}} g^{*} \cdot v \mathrm{~d} x+\int_{\partial \Omega \cap U_{0}} g_{2}^{*} \cdot v \mathrm{~d} \sigma \quad \forall v \in X, \\
\nabla \cdot z=g_{1}^{*} \text { in } \Omega \cap U_{0} .
\end{array}\right.
$$


Here, we have denoted

$$
X=\left\{v \in H^{1}\left(\Omega \cap U_{0}\right)^{N}: v=0 \text { on } \partial U_{0} \cap \Omega, v \cdot n=0 \text { on } \partial \Omega \cap U_{0}\right\} .
$$

Let us now perform the change of variables $x=\psi(\xi)$. If we define $\tilde{z}=z \circ \psi$ and $\tilde{h}=h \circ \psi$, they verify:

$$
\left\{\begin{array}{l}
\sum_{i, j} \int_{C_{0}^{+}}\left(\nabla \tilde{z}^{i} \cdot \partial_{j} \psi^{-1}+\nabla \tilde{z}^{j} \cdot \partial_{i} \psi^{-1}\right)\left(\nabla \tilde{v}^{i} \cdot \partial_{j} \psi^{-1}\right)(\xi)|J(\psi)|(\xi) \mathrm{d} \xi \\
-\int_{C_{0}^{+}} \tilde{h}\left(\nabla \tilde{v}: \nabla^{t} \psi^{-1}\right)(\xi)|J(\psi)|(\xi) \mathrm{d} \xi=\int_{C_{0}^{+}}\left(\tilde{g}^{*} \cdot \tilde{v}\right)(\xi)|J(\psi)|(\xi) \mathrm{d} \xi \\
+\int_{\Delta_{\alpha_{0}}}\left(\tilde{g}_{2}^{*} \cdot \tilde{v}\right)\left(\xi^{\prime}, 0\right)|J(\psi)|\left(\xi^{\prime}, 0\right) \mathrm{d} \xi^{\prime} \quad \forall \tilde{v} \in \tilde{X}, \\
\nabla \tilde{z}: \nabla^{t} \psi^{-1}=\tilde{g}_{1}^{*} \text { in } C_{0}^{+},
\end{array}\right.
$$

with

and where we have denoted

$$
\tilde{X}=\left\{\tilde{v} \in H^{1}\left(C_{0}^{+}\right)^{N}: \tilde{v}=0 \quad \text { on } \partial C_{0}^{+} \cap \mathbf{R}_{+}^{N}, \tilde{v} \cdot n=0 \text { on } \Delta_{\alpha_{0}}\right\}
$$

and $|J(\psi)|$ the determinant of the Jacobian of $\psi$.

$$
\tilde{g}^{*}=g^{*} \circ \psi, \quad \tilde{g}_{1}^{*}=g_{1}^{*} \circ \psi, \quad \tilde{g}_{2}^{*}=g_{2}^{*} \circ \psi
$$

Let us introduce $C_{1}=\psi^{-1}\left(U_{1}\right)$ and $d=\operatorname{dist}\left(\partial C_{0}^{+}, \partial C_{1}^{+}\right)$. Then, for every function $\tilde{v} \in H^{1}\left(C_{1}^{+}\right)^{N}$ verifying

$$
\tilde{v}=0 \text { on } \partial C_{1}^{+} \cap \mathbf{R}_{+}^{N} \quad \text { and } \tilde{v} \cdot n \text { on } \partial C_{1}^{+} \cap \partial \mathbf{R}_{+}^{N}=\Delta_{\alpha_{1}}
$$

(that is to say, $\tilde{v} \in \tilde{X}_{1}$ ), we have $\delta_{k}^{-m} \tilde{v} \in \tilde{X}$, for $k \in\{1, \ldots, N-1\}$ and $|m| \leq d / 2$. We remind that, by definition,

$$
\delta_{k}^{m}(x)=\frac{v\left(x+m e_{k}\right)-v(x)}{m}
$$

This allows us to plug $\delta_{k}^{-m} \tilde{v}$ into expression (28):

$$
\left\{\begin{array}{l}
\sum_{i, j} \int_{C_{1}^{+}} \delta_{k}^{m}\left[|J(\psi)|\left(\nabla \tilde{z}^{i} \cdot \partial_{j} \psi^{-1}+\nabla \tilde{z}^{j} \cdot \partial_{i} \psi^{-1}\right) \partial_{j} \psi^{-1}\right] \cdot \nabla \tilde{v}^{i} \mathrm{~d} \xi \\
-\int_{C_{1}^{+}} \delta_{k}^{m}\left(|J(\psi)| \tilde{h} \nabla^{t} \psi^{-1}\right): \nabla \tilde{v} \mathrm{~d} \xi \\
\quad=\int_{C_{1}^{+}} \delta_{k}^{m}\left(|J(\psi)| \tilde{g}^{*}\right) \cdot \tilde{v} \mathrm{~d} \xi+\int_{\Delta_{\alpha_{1}}} \delta_{k}^{m}\left(|J(\psi)| \tilde{g}_{2}^{*}\right) \cdot \tilde{v} \mathrm{~d} \xi^{\prime} \quad \forall \tilde{v} \in \tilde{X}_{1} \\
\nabla \tilde{z}: \nabla^{t} \psi^{-1}=\tilde{g}_{1}^{*} \text { in } C_{1}^{+} .
\end{array}\right.
$$

Let us compute each one of the previous integrals, taking into account (25) and the formula:

$$
\delta_{k}^{m}\left(v_{1} v_{2}\right)(x)=v_{1}(x) \delta_{k}^{m} v_{2}(x)+\delta_{k}^{m} v_{1}(x) v_{2}\left(x+m e_{k}\right) .
$$

First, we have

$$
\begin{aligned}
& \sum_{i, j} \int_{C_{1}^{+}} \delta_{k}^{m}\left[|J(\psi)|\left(\nabla \tilde{z}^{i} \cdot \partial_{j} \psi^{-1}+\nabla \tilde{z}^{j} \cdot \partial_{i} \psi^{-1}\right) \partial_{j} \psi^{-1}\right] \cdot \nabla \tilde{v}^{i} \mathrm{~d} \xi \\
& =\sum_{i, j} \int_{C_{1}^{+}}|J(\psi)|\left(\nabla\left(\delta_{k}^{m} \tilde{z}^{i}\right) \cdot \partial_{j} \psi^{-1}+\nabla\left(\delta_{k}^{m} \tilde{z}^{j}\right) \cdot \partial_{i} \psi^{-1}\right)\left(\nabla \tilde{v}^{i} \cdot \partial_{j} \psi^{-1}\right) \mathrm{d} \xi+I_{1}
\end{aligned}
$$


with

Additionally, we find

$$
\begin{aligned}
& \left|I_{1}\right| \leq C\|\psi\|_{W^{2, \infty}\left(C_{0}^{+}\right)^{N}}\|\tilde{z}\|_{H^{1}\left(C_{1}^{+}\right)^{N}}\|\tilde{v}\|_{H^{1}\left(C_{1}^{+}\right)^{N}} \\
& \leq C\left(\|g\|_{W^{\prime}}+\left\|f_{3}\right\|_{H^{-1 / 2}(\partial \Omega)^{N}}\right)\|\tilde{v}\|_{H^{1}\left(C_{1}^{+}\right)^{N}} .
\end{aligned}
$$

$$
-\int_{C_{1}^{+}} \delta_{k}^{m}\left(|J(\psi)| \tilde{h} \nabla^{t} \psi^{-1}\right): \nabla \tilde{v} \mathrm{~d} \xi=-\int_{C_{1}^{+}}|J(\psi)| \delta_{k}^{m} \tilde{h} \nabla^{t} \psi^{-1}: \nabla \tilde{v} \mathrm{~d} \xi+I_{2},
$$

with

$$
\left|I_{2}\right| \leq C\|\tilde{h}\|_{L^{2}\left(C_{1}^{+}\right)}\|\tilde{v}\|_{H^{1}\left(C_{1}^{+}\right)^{N}} \leq C\left(\|g\|_{W^{\prime}}+\left\|f_{3}\right\|_{H^{-1 / 2}(\partial \Omega)^{N}}\right)\|\tilde{v}\|_{H^{1}\left(C_{1}^{+}\right)^{N}} .
$$

Furthermore,

$$
I_{3}=\int_{C_{1}^{+}} \delta_{k}^{m}\left(|J(\psi)| \tilde{g}^{*}\right) \cdot \tilde{v} \mathrm{~d} \xi \leq C\left\|\tilde{g}^{*}\right\|_{L^{2}\left(C_{1}^{+}\right)^{N}}\|\tilde{v}\|_{\tilde{X}_{1}} \leq C\left(\|g\|_{L^{2}(\Omega)^{N}}+\left\|f_{3}\right\|_{H^{-1 / 2}(\partial \Omega)^{N}}\right)\|\tilde{v}\|_{H^{1}\left(C_{1}^{+}\right)^{N}}
$$

where we have denoted

$$
\|\tilde{v}\|_{\tilde{X}_{1}}^{2}=\sum_{j=1}^{N-1} \int_{C_{1}^{+}}\left|\partial_{j} \tilde{v}\right|^{2} \mathrm{~d} x+\int_{C_{1}^{+}}\left|\partial_{N} \tilde{v}+\nabla \tilde{v}_{N}\right|^{2} \mathrm{~d} x .
$$

Finally, we have

$$
\begin{aligned}
I_{4}=\int_{\Delta_{\alpha_{1}}} \delta_{k}^{m}\left(|J(\psi)| \tilde{g}_{2}^{*}\right) \cdot \tilde{v} \mathrm{~d} \xi^{\prime}= & \int_{\Delta_{\alpha_{1}+d / 2}} \delta_{k}^{m}\left(|J(\psi)| \tilde{g}_{2}^{*}\right) \cdot \tilde{v} \mathrm{~d} \xi^{\prime} \\
& \leq C\left\|\delta_{h}^{m} \tilde{g}_{2}^{*}\right\|_{H_{00}^{1 / 2}\left(\Delta_{\alpha_{1}+d / 2}\right)}\|\tilde{v}\|_{H_{00}^{1 / 2}\left(\Delta_{\alpha_{1}+d / 2}\right)} \\
& \leq C\left\|\tilde{g}_{2}^{*}\right\|_{H^{1 / 2}\left(\Delta_{\alpha_{1}}\right)^{N}}\|\tilde{v}\|_{H^{1 / 2}\left(\Delta_{\alpha_{1}}\right)^{N}} \\
& \leq C\left(\|g\|_{L^{2}(\Omega)^{N}}+\left\|f_{3}\right\|_{H^{1 / 2}(\partial \Omega)^{N}}\right)\|\tilde{v}\|_{H^{1}\left(C_{1}^{+}\right)^{N}}
\end{aligned}
$$

Here, we have employed the notation

$$
H_{00}^{1 / 2}(\Delta)=\left\{v \in H^{1 / 2}(\Delta): \rho_{\Delta}^{-1 / 2} v \in L^{2}(\Delta)\right\} \quad \text { with } \quad \rho_{\Delta}(x)=\operatorname{dist}(x, \partial \Delta)
$$

and we have used the fact that

$$
\frac{\partial}{\partial x_{i}} \in \mathcal{L}\left(H^{1 / 2},\left(H_{00}^{1 / 2}\right)^{\prime}\right)
$$

(see [16] for more details).

Consequently, we obtain an equivalent formulation of (29):

$$
\left\{\begin{array}{l}
a_{0}\left(\delta_{k}^{m} \tilde{z}, \tilde{v}\right)+b_{0}\left(\tilde{v}, \delta_{k}^{m} \tilde{h}\right)=-\left(I_{1}+I_{2}+I_{3}+I_{4}\right) \quad \forall \tilde{v} \in \tilde{X}_{1}, \\
b_{0}\left(\delta_{k}^{m} \tilde{z}, \tilde{f}_{0}\right)=-I_{5} \quad \forall \tilde{f}_{0} \in L^{2}\left(C_{1}^{+}\right),
\end{array}\right.
$$

with

$$
\begin{gathered}
a_{0}\left(v_{1}, v_{2}\right)=\sum_{i, j} \int_{C_{1}^{+}}|J(\psi)|\left(\nabla v_{1}^{i} \cdot \partial_{j} \psi^{-1}+\nabla v_{1}^{j} \cdot \partial_{i} \psi^{-1}\right)\left(\partial_{j} \psi^{-1} \cdot \nabla v_{2}^{i}\right) \mathrm{d} \xi \\
b_{0}\left(v_{2}, v_{3}\right)=-\int_{C_{1}^{+}}|J(\psi)| v_{3}\left(\nabla^{t} \psi^{-1}: \nabla v_{2}\right)
\end{gathered}
$$


for $v_{1}, v_{2} \in \tilde{X}_{1}$ and $v_{3} \in L^{2}\left(C_{1}^{+}\right)$and

$$
I_{5}=\int_{C_{1}^{+}}|J(\psi)| \tilde{f}_{0}\left(\nabla^{t} \psi^{-1}: \nabla\left(\delta_{k}^{m} \tilde{z}\right)\right) \mathrm{d} \xi
$$

From the last condition in (29), we find

$$
I_{5}=\int_{C_{1}^{+}}|J(\psi)| \tilde{f}_{0} \delta_{k}^{m} \tilde{g}_{1}^{*} \mathrm{~d} \xi-\int_{C_{1}^{+}}|J(\psi)| \tilde{f}_{0}\left(\delta_{k}^{m}\left(\nabla \psi^{-1}\right): \nabla \tilde{z}\left(\xi+m e_{k}\right)\right) \mathrm{d} \xi
$$

so

$$
\begin{aligned}
& \left|I_{5}\right| \leq C\left(\left\|\tilde{g}_{1}^{*}\right\|_{H^{1}\left(C_{1}^{+}\right)}+\|\tilde{z}\|_{H^{1}\left(C_{1}^{+}\right)^{N}}\right)\left\|\tilde{f}_{0}\right\|_{L^{2}\left(C_{1}^{+}\right)} \\
& \leq C\left(\|g\|_{W^{\prime}}+\left\|f_{3}\right\|_{H^{-1 / 2}(\partial \Omega)^{N}}\right)\left\|\tilde{f}_{0}\right\|_{L^{2}\left(C_{1}^{+}\right)^{+}}
\end{aligned}
$$

The mixed problem (29) will posses a unique solution $\left(\delta_{k}^{m} \tilde{z}, \delta_{k}^{m} \tilde{h}\right) \in \tilde{X}_{1} \times L^{2}\left(C_{1}^{+}\right)$if we prove that $a_{0}$ is continuous and coercive in $\tilde{X}_{1}$ and that $b_{0}$ is continuous and verifies the inf-sup condition in $\tilde{X}_{1} \times L^{2}\left(C_{1}^{+}\right)$.

The continuity of $a_{0}$ and $b_{0}$ are trivial. Let us prove that $a$ is coercive; for $\tilde{v}_{1} \in \tilde{X}_{1}$, we have

$$
\begin{aligned}
a_{0}\left(\tilde{v}_{1}, \tilde{v}_{1}\right) & =\sum_{i, j} \int_{C_{1}^{+}}|J(\psi)|\left(\nabla \tilde{v}_{1}^{i} \cdot \partial_{j} \psi^{-1}+\nabla \tilde{v}_{1}^{j} \cdot \partial_{i} \psi^{-1}\right)\left(\nabla \tilde{v}_{1}^{i} \cdot \partial_{j} \psi^{-1}\right) \mathrm{d} \xi \\
& =\frac{1}{2} \sum_{i, j} \int_{C_{1}^{+}}|J(\psi)|\left|\nabla \tilde{v}_{1}^{i} \cdot \partial_{j} \psi^{-1}+\nabla \tilde{v}_{1}^{j} \cdot \partial_{i} \psi^{-1}\right|^{2} \mathrm{~d} \xi=\frac{1}{2} \int_{\Omega \cap U_{1}}\left|D v_{1}\right|^{2} \mathrm{~d} x \\
& \geq C\left\|v_{1}\right\|_{H^{1}\left(\Omega \cap U_{1}\right)^{N}}^{2} \geq \tilde{C}\left\|\tilde{v}_{1}\right\|_{H^{1}\left(C_{1}^{+}\right)^{N}}^{2}
\end{aligned}
$$

Finally, the inf-sup condition for $b_{0}$ tells that

$$
\sup _{\tilde{v} \in \tilde{X}_{1} \backslash\{0\}} \frac{b_{0}\left(\tilde{v}, \tilde{f}_{0}\right)}{\|\tilde{v}\|_{H^{1}\left(C_{1}^{+}\right)^{N}}} \geq C_{2}\left\|\tilde{f}_{0}\right\|_{L^{2}\left(C_{1}^{+}\right)} \quad \forall \tilde{f}_{0} \in L^{2}\left(C_{1}^{+}\right) .
$$

To prove this, we first observe that

$$
b_{0}\left(\tilde{v}, \tilde{f}_{0}\right)=\int_{\Omega \cap U_{1}} f_{0} \nabla \cdot v \mathrm{~d} x
$$

Now, for $f_{0} \in L^{2}\left(\Omega \cap U_{1}\right) \backslash\{0\}$, we consider $v \in H_{0}^{1}\left(\Omega \cap U_{1}\right)^{N}$ such that $\nabla \cdot v=f_{0}$ and

$$
\|v\|_{H^{1}\left(\Omega \cap U_{1}\right)^{N}} \leq C_{3}\left\|f_{0}\right\|_{L^{2}\left(\Omega \cap U_{1}\right)}
$$

(see, for instance, [11]). Consequently,

$$
\begin{aligned}
& \sup _{\substack{\tilde{v} \in \tilde{X}_{1} \backslash\{0\} \\
\geq}} \frac{b_{0}\left(\tilde{v}, \tilde{f}_{0}\right)}{\|\tilde{v}\|_{H^{1}\left(C_{1}^{+}\right)^{N}}} \geq C_{4} \frac{\int_{\Omega \cap U_{1}} f_{0} \nabla \cdot v \mathrm{~d} x}{\|v\|_{H^{1}\left(\Omega \cap U_{1}\right)^{N}}}=C_{4} \frac{\left\|f_{0}\right\|_{L^{2}\left(\Omega \cap U_{1}\right)}^{2}}{\|v\|_{H^{1}\left(\Omega \cap U_{1}\right)^{N}}} \\
& \geq C_{3}^{-1} C_{4}\left\|f_{0}\right\|_{L^{2}\left(\Omega \cap U_{1}\right)} \geq C_{2}\left\|\tilde{f}_{0}\right\|_{L^{2}\left(C_{1}^{+}\right)},
\end{aligned}
$$

as we wanted to see.

As a conclusion, there exists a unique solution $\left(\delta_{k}^{m} \tilde{z}, \delta_{k}^{m} \tilde{h}\right) \in \tilde{X}_{1} \times L^{2}\left(C_{1}^{+}\right)$of $(30)$ and

$$
\left\|\delta_{k}^{m} \tilde{z}\right\|_{\tilde{X}_{1}}+\left\|\delta_{k}^{m} \tilde{h}\right\|_{L^{2}\left(C_{1}^{+}\right)} \leq C\left(\|g\|_{L^{2}(\Omega)^{N}}+\left\|f_{3}\right\|_{H^{1 / 2}(\partial \Omega)^{N}}\right)
$$


for $k \in\{1, \ldots, N-1\}$. This tells that $\left(\partial_{k} \tilde{z}, \partial_{k} \tilde{h}\right) \in H^{1}\left(C_{1}^{+}\right)^{N} \times L^{2}\left(C_{1}^{+}\right)$, so that $\left(\partial_{k} z, \partial_{k} h\right) \in H^{1}(\Omega \cap \tilde{U})^{N} \times$ $L^{2}(\Omega \cap \tilde{U})$. Finally, from the divergence free condition (in $\left.\Omega \cap \tilde{U}\right)$ and the differential equation verified by $(z, h)$, we have $\partial_{N N} z \in L^{2}(\Omega \cap \tilde{U})^{N}$ which implies that $\partial_{N} h \in L^{2}(\Omega \cap \tilde{U})$.

Therefore, $(u, \theta) \in H^{2}(\Omega \cap \tilde{U})^{N} \times H^{1}(\Omega \cap \tilde{U})$ for every $\tilde{U} \subset \subset U$ and

$$
\|u\|_{H^{2}(\Omega \cap \tilde{U})^{N}}+\|\theta\|_{H^{1}(\Omega \cap \tilde{U})} \leq C\left(\|g\|_{L^{2}(\Omega)^{N}}+\left\|f_{3}\right\|_{H^{1 / 2}(\partial \Omega)^{N}}\right) .
$$

Combining this estimate with the local one (26), we obtain the estimate for the solution of (23), say:

$$
\begin{aligned}
& \|u(t)\|_{H^{2}(\Omega)^{N}}+\|\theta(t)\|_{H^{1}(\Omega)} \leq\left(\left\|f_{1}(t)\right\|_{L^{2}(\Omega)^{N}}+\left\|u_{t}(t)\right\|_{L^{2}(\Omega)^{N}}\right. \\
& \left.\quad+\left\|f_{2}(t)\right\|_{H^{1 / 2}(\partial \Omega)^{N}}+\|A u(t)\|_{H^{1 / 2}(\partial \Omega)^{N}}\right) \quad \text { a.e. } t \in(0, T) .
\end{aligned}
$$

Let us now put this estimate together with (22). We obtain

$$
\begin{aligned}
\left\|u_{t}(t)\right\|_{L^{2}(\Omega)^{N}}^{2}+\|u(t)\|_{H^{2}(\Omega)^{N}}^{2}+\frac{\mathrm{d}}{\mathrm{d} t} \int_{\Omega}|D u(t)|^{2} \mathrm{~d} x+\|\theta(t)\|_{H^{1}(\Omega)}^{2} & \\
\leq C\left(\left\|f_{1}(t)\right\|_{L^{2}(\Omega)^{N}}^{2} \mathrm{~d} x+\left\|f_{2}(t)\right\|_{H^{1 / 2}(\partial \Omega)^{N}}^{2}+\left|\left\langle f_{2}(t), u_{t}(t)\right\rangle_{\partial \Omega}\right|\right. & \\
& \left.+\|A u(t)\|_{H^{1 / 2}(\partial \Omega)^{N}}^{2}+\int_{\partial \Omega}\left|A u(t) \| u_{t}(t)\right| \mathrm{d} \sigma\right),
\end{aligned}
$$

for almost every $t \in(0, T)$. A classical argument based on Gronwall's lemma leads to the absorption of the fourth term in the right hand side:

$$
\begin{aligned}
\left\|u_{t}\right\|_{L^{2}(Q)^{N}}^{2}+\|u\|_{L^{2}\left(H^{2}\right)}^{2}+ & \|u\|_{L^{\infty}\left(H^{1}\right)}^{2}+\|\theta\|_{L^{2}\left(H^{1}\right)}^{2} \\
& \leq C \mathrm{e}^{C T\|A\|_{L^{\infty}\left(W^{1, \nu}\right)}^{2}}\left(\left\|f_{1}\right\|_{L^{2}(Q)^{N}}^{2} \mathrm{~d} x+\left\|f_{2}\right\|_{L^{2}\left(H^{1 / 2}\right)}^{2}+\left\|u^{0}\right\|_{H^{1}(\Omega)^{N}}^{2}\right. \\
& \left.\quad+\int_{0}^{T}\left|\left\langle f_{2}(t), u_{t}(t)\right\rangle_{\partial \Omega}\right| \mathrm{d} t+\iint_{\Sigma}\left|A\|u\| \partial_{t} u\right| \mathrm{d} \sigma \mathrm{d} t\right)
\end{aligned}
$$

with $\nu>N-1$ arbitrarily small. Indeed, this readily follows from the fact that

$$
W^{1, \nu}(\partial \Omega) \cdot H^{1 / 2}(\partial \Omega) \subset H^{1 / 2}(\partial \Omega) \quad \text { continuously, }
$$

that is to say, the product of a function belonging to $W^{1, \nu}(\partial \Omega)$ and another one belonging to $H^{1 / 2}(\partial \Omega)$ actually belongs to $H^{1 / 2}(\partial \Omega)$.

In order to estimate the last term on (31), observe that for a vector valued function $e^{0}$ verifying $e_{t}^{0} \in L^{2}(Q)^{N}$ and $e^{0} \in L^{2}\left(0, T ; H^{1}(\Omega)^{N}\right)$, we have

$$
e^{0} \in H^{\ell^{\prime}}\left(0, T ; H^{1-\ell^{\prime}}(\Omega)^{N}\right)
$$

for every $0<\ell^{\prime}<1$ and there exists a constant $C>0$ such that

$$
\left\|e^{0}\right\|_{H^{\ell^{\prime}\left(H^{1-\ell^{\prime}}\right)}} \leq C\left\|e_{t}^{0}\right\|_{L^{2}(Q)^{N}}^{\ell^{\prime}}\left\|e^{0}\right\|_{L^{2}\left(H^{1}\right)}^{1-\ell^{\prime}} .
$$

In particular, one can check that for some $0<\ell^{\prime}<1$

$$
\left\langle\partial_{t}\left(\gamma_{0} e^{0}\right), e^{1}\left(\gamma_{0} e^{0}\right)\right\rangle_{\partial \Omega}<+\infty
$$


for $e^{1} \in H^{1-\ell}\left(0, T ; H^{\nu / 2}(\partial \Omega)^{N}\right)$; here, we have denoted by $\langle\cdot, \cdot\rangle_{\partial \Omega}$ the duality product between the spaces $H^{\ell^{\prime}-1}\left(0, T ; H^{1 / 2-\ell^{\prime}}(\partial \Omega)\right)$ and $H^{1-\ell^{\prime}}\left(0, T ; H^{\ell^{\prime}-1 / 2}(\partial \Omega)\right)$ and by $\gamma_{0}\left(e_{0}\right)$ the trace of $e_{0}$ on the boundary $\partial \Omega$. Besides, we get

$$
\left|\left\langle\partial_{t}\left(\gamma_{0} e^{0}\right), e^{1}\left(\gamma_{0} e^{0}\right)\right\rangle_{\partial \Omega}\right| \leq C\left\|e^{1}\right\|_{H^{1-\ell}\left(H^{\nu / 2}\right)}\left\|e_{t}^{0}\right\|_{L^{2}(Q)^{N}}\left\|e^{0}\right\|_{L^{2}\left(H^{1}\right)}
$$

This way, the last term in (31) can be estimated as follows:

$$
\iint_{\Sigma}\left|A\|u\| \partial_{t} u\right| \mathrm{d} \sigma \mathrm{d} t \leq \varepsilon\left\|u_{t}\right\|_{L^{2}(Q)^{N}}^{2}+C\left(1+\|A\|_{H^{1-\ell}\left(H^{\left.-3 / 2+N / 2+2 \ell^{\prime}\right)}\right.}^{2}\right)\|u\|_{L^{2}\left(H^{1}\right)}^{2},
$$

for a small positive constant $\varepsilon(\Omega)$. Then,

$$
\begin{aligned}
\left\|u_{t}\right\|_{L^{2}(Q)^{N}}^{2}+\|u\|_{L^{2}\left(H^{2}\right)}^{2}+ & \|u\|_{L^{\infty}\left(H^{1}\right)}^{2}+\|\theta\|_{L^{2}\left(H^{1}\right)}^{2} \\
\leq C \mathrm{e}^{C T\|A\|_{L^{\infty}\left(W^{1, \nu}\right)}^{2}} & \left(\left\|f_{1}\right\|_{L^{2}(Q)^{N}}^{2}+\left\|f_{2}\right\|_{L^{2}\left(H^{1 / 2}\right)}^{2}+\left\|u^{0}\right\|_{H^{1}(\Omega)^{N}}^{2}\right. \\
& \left.+\int_{0}^{T}\left|\left\langle f_{2}(t), u_{t}(t)\right\rangle_{\partial \Omega}\right| \mathrm{d} t+\left(1+\|A\|_{H^{1-\ell}\left(H^{\nu / 2}\right)}^{2}\right)\|u\|_{L^{2}\left(H^{1}\right)}^{2}\right)
\end{aligned}
$$

which, combined with (20), yields

$$
\begin{aligned}
\left\|u_{t}\right\|_{L^{2}(Q)^{N}}^{2}+\|u\|_{L^{2}\left(H^{2}\right)}^{2}+ & \|u\|_{L^{\infty}\left(H^{1}\right)}^{2}+\|\theta\|_{L^{2}\left(H^{1}\right)}^{2} \\
& \leq C \mathrm{e}^{C T\|A\|_{H^{1-\ell}\left(W^{\nu_{1}, \nu_{1}+1}\right)}^{2}\left(1+\|A\|_{H^{1-\ell}\left(W^{\left.1, \nu_{1}\right)}\right)}^{2}\right)\left(\left\|f_{1}\right\|_{L^{2}(Q)^{N}}^{2}\right.} \\
& \left.+\left\|f_{2}\right\|_{L^{2}\left(H^{1 / 2}\right)}^{2}+\left\|u^{0}\right\|_{H^{1}(\Omega)^{N}}^{2}+\int_{0}^{T}\left|\left\langle f_{2}(t), u_{t}(t)\right\rangle_{\partial \Omega}\right| \mathrm{d} t\right)
\end{aligned}
$$

Here, we have used the fact (recall that $\ell$ is close to $1 / 2$ and the definition of $\nu_{1}$ )

$$
H^{1-\ell}\left(0, T ; W^{\nu_{1}, \nu_{1}+1}(\partial \Omega)\right) \subset L^{\infty}\left(0, T, W^{1, \nu} \cap H^{\nu / 2}(\partial \Omega)\right) \text { continuously. }
$$

Let us now combine $u_{t} \in L^{2}(Q)^{N}$ and $u \in L^{2}\left(0, T ; H^{2}(\Omega)^{N}\right)$ to obtain an estimate of $\left\langle f_{2}, u_{t}\right\rangle_{\partial \Omega}$ in appropriate spaces. More precisely, we have

$$
u \in H^{(1+\ell) / 2}\left(0, T ; H^{1-\ell}(\Omega)^{N}\right)
$$

and

We find:

$$
\|u\|_{H^{(1+\ell) / 2}\left(H^{1-\ell}\right)} \leq C\left\|u_{t}\right\|_{L^{2}(Q)^{N}}^{(1+\ell) / 2}\|u\|_{L^{2}\left(H^{2}\right)}^{(1-\ell) / 2} .
$$

$$
\begin{aligned}
\int_{0}^{T}\left|\left\langle f_{2}(t), u_{t}(t)\right\rangle_{\partial \Omega}\right| \mathrm{d} t \leq & C\left\|f_{2}\right\|_{H^{(1-\ell) / 2}\left(H^{\ell-1 / 2}\right)}\left\|u_{t}\right\|_{L^{2}(Q)^{N}}^{(1+\ell) / 2}\|u\|_{L^{2}\left(H^{2}\right)}^{(1-\ell) / 2} \\
\leq & C\left(1+\|A\|_{H^{1-\ell}\left(W^{\nu_{1}, \nu_{1}+1}\right)}^{2}\right)\left\|f_{2}\right\|_{H^{(1-\ell) / 2}\left(H^{\ell-1 / 2}\right)}^{2} \\
& +\varepsilon_{1}\left(1+\|A\|_{H^{1-\ell}\left(W^{\nu_{1}, \nu_{1}+1}\right)}^{2}\right)^{-1}\left\|u_{t}\right\|_{L^{2}(Q) N}^{1+\ell}\|u\|_{L^{2}\left(H^{2}\right)}^{1-\ell} \\
\leq & C\left(1+\|A\|_{H^{1-\ell}\left(W^{\nu_{1}, \nu_{1}+1}\right)}^{2}\right)\left\|f_{2}\right\|_{H^{(1-\ell) / 2}\left(H^{\ell-1 / 2}\right)}^{2} \\
& +\varepsilon_{2}\left(1+\|A\|_{H^{1-\ell}\left(W^{\nu_{1}, \nu_{1}+1}\right)}^{2}\right)^{-1}\left(\left\|u_{t}\right\|_{L^{2}(Q)^{N}}^{2}+\|u\|_{L^{2}\left(H^{2}\right)}^{2}\right)
\end{aligned}
$$


where $\varepsilon_{1}$ and $\varepsilon_{2}$ are small positive constants depending on $\Omega$ and $\ell$. Plugging this into (34), we find

$$
\begin{aligned}
& \left\|u_{t}\right\|_{L^{2}(Q)^{N}}^{2}+\|u\|_{L^{2}\left(H^{2} \cap W\right)}^{2}+\|u\|_{L^{\infty}\left(H^{1}\right)}^{2}+\|\theta\|_{L^{2}\left(H^{1}\right)}^{2} \\
& \quad \leq C \mathrm{e}^{C T\|A\|_{H^{1-\ell}\left(W^{\nu_{1}, \nu_{1}+1}\right)}^{2}\left(1+\|A\|_{H^{1-\ell}\left(W^{\nu_{1}, \nu_{1}+1}\right)}^{4}\right)\left(\left\|f_{1}\right\|_{L^{2}(Q)^{N}}^{2}\right.}+ \\
& \left.\quad+\left\|f_{2}\right\|_{L^{2}\left(H^{1 / 2}\right)}^{2}+\left\|f_{2}\right\|_{H^{(1-\ell) / 2}\left(H^{\ell-1 / 2}\right)}^{2}+\left\|u^{0}\right\|_{H^{1}(\Omega)^{N}}^{2}\right),
\end{aligned}
$$

which is exactly (18).

This ends the proof of Proposition 1.1.

Finally, we establish a further regularity result when the data is supposed to be more regular. This will be used when proving the local null controllability to the trajectories of system (1) in the last section. More precisely, it concerns a linear Stokes system similar to (16) but with null $f_{2}$ :

$$
\begin{cases}u_{t}-\nabla \cdot(D u)+\nabla \theta=f_{4} & \text { in } Q, \\ \nabla \cdot u=0 & \text { in } Q, \\ u \cdot n=0,(\sigma(u, \theta) \cdot n)_{t g}+(A(x, t) u)_{t g}=0 & \text { on } \Sigma, \\ u(\cdot, 0)=u^{0}(\cdot) & \text { in } \Omega .\end{cases}
$$

Proposition 1.2. Let $f_{4} \in H^{1}\left(0, T ; L^{2}(\Omega)^{N}\right) \cap C\left([0, T] ; H^{1}(\Omega)^{N}\right)$ with $f_{4} \cdot n=0, u^{0} \in H^{3}(\Omega)^{N} \cap W$ satisfying the compatibility condition

$$
\left(D u^{0} \cdot n\right)_{t g}+\left(A(x, 0) u^{0}\right)_{t g}=0 \text { on } \partial \Omega
$$

and let A satisfy (8)-(10).

Then, the strong solution $u$ of (37) actually verifies $u \in H^{1}\left(0, T ; H^{2}(\Omega)^{N} \cap W\right) \cap H^{2}(0, T ; H)$ and

$$
\|u\|_{H^{1}\left(H^{2} \cap W\right)}^{2}+\|u\|_{H^{2}\left(L^{2}\right)}^{2} \leq C(\Omega, A)\left(\left\|f_{4}\right\|_{H^{1}\left(L^{2}\right) \cap L^{\infty}\left(H^{1}\right)}^{2}+\left\|u^{0}\right\|_{H^{3} \cap W}^{2}\right),
$$

where $C$ is a positive constant.

Proof. From proposition 1.1, we already know that $u$ is a strong solution of (37) (i.e., it verifies (17)). In particular, $u \in H^{1}(0, T ; H)$ and

$$
\|u\|_{H^{1}\left(L^{2}\right)}^{2} \leq C\left(\Omega,\|A\|_{P}\right)\left(\left\|u^{0}\right\|_{W}^{2}+\left\|f_{4}\right\|_{L^{2}(Q)^{N}}^{2}\right) .
$$

Next, we are going to give sense to $\partial_{t} u$ as the strong solution of the following system:

$$
\begin{cases}\tilde{u}_{t}-\nabla \cdot(D \tilde{u})+\nabla \tilde{\theta}=\partial_{t} f_{4} & \text { in } Q, \\ \nabla \cdot \tilde{u}=0 & \text { in } Q, \\ \tilde{u} \cdot n=0,(D \tilde{u} \cdot n)_{t g}+(A(x, t) \tilde{u})_{t g}=-(A(x, t))_{t} u & \text { on } \Sigma, \\ \tilde{u}(\cdot, 0)=\partial_{t} u(\cdot, 0) & \text { in } \Omega .\end{cases}
$$

To this end, we must first check that

$$
A_{t} \gamma_{0} u \in L^{2}\left(0, T ; H^{-1 / 2}(\partial \Omega)^{N}\right)
$$

and

From the fact that

$$
\partial_{t} u(\cdot, 0) \in H
$$

$$
u \in L^{2}\left(0, T ; H^{2}(\Omega)^{N}\right) \cap H^{1}\left(0, T ; L^{2}(\Omega)^{N}\right) \subset H^{1-\ell}\left(0, T ; H^{2 \ell-1 / 2}(\partial \Omega)^{N}\right)
$$


continuously and (10), we have

$$
\begin{gathered}
A_{t} \gamma_{0} u \in L^{2}\left(0, T ; H^{1-2 \ell}(\partial \Omega)^{N^{2}}\right) \cdot H^{1-\ell}\left(0, T ; H^{2 \ell-1 / 2}(\partial \Omega)^{N}\right) \\
\subset L^{2}\left(0, T ; H^{-1 / 2}(\partial \Omega)^{N}\right) \text { continuously }
\end{gathered}
$$

(recall that this notation was already used in (32)) so

$$
\left\|A_{t} \gamma_{0} u\right\|_{L^{2}\left(H^{-1 / 2}\right)}^{2} \leq C\|A\|_{H^{1}\left(H^{1-2 \ell}\right)}^{2}\left(\|u\|_{H^{1}\left(L^{2}\right)}^{2}+\|u\|_{L^{2}\left(H^{2}\right)}^{2}\right) .
$$

In order to prove (41), we use the differential equation in (37) and we find

$$
\begin{aligned}
\int_{\Omega}\left|\partial_{t} u(0)\right|^{2} \mathrm{~d} x & =\int_{\Omega} \partial_{t} u(0) \cdot\left(\nabla \cdot\left(D u^{0}\right)-\nabla \theta(0)+f_{4}(0)\right) \mathrm{d} x \\
& =\int_{\Omega} \partial_{t} u(0) \cdot\left(\nabla \cdot\left(D u^{0}\right)+f_{4}(0)\right) \mathrm{d} x \\
& \leq \frac{1}{2} \int_{\Omega}\left|\partial_{t} u(0)\right|^{2} \mathrm{~d} x+C\left(\left\|u^{0}\right\|_{H^{2} \cap W}^{2}+\left\|f_{4}\right\|_{L^{\infty}\left(L^{2}\right)}^{2}\right)
\end{aligned}
$$

Consequently, we have that (40) has a unique weak solution $\tilde{u} \in L^{2}(0, T ; W) \cap L^{\infty}(0, T ; H)$, which must coincide with $\partial_{t} u$. Therefore, $\partial_{t} u \in L^{2}(0, T ; W) \cap L^{\infty}(0, T ; H)$ and

$$
\left\|\partial_{t} u\right\|_{L^{2}\left(H^{1}\right) \cap L^{\infty}\left(L^{2}\right)}^{2} \leq C(\Omega, A)\left(\left\|f_{4}\right\|_{H^{1}\left(L^{2}\right)}^{2}+\left\|u^{0}\right\|_{H^{2} \cap W}^{2}\right) .
$$

Finally and by virtue of Proposition 1.1, we must check that

$$
A_{t} \gamma_{0} u \in L^{2}\left(0, T ; H^{1 / 2}(\partial \Omega)^{N}\right) \cap H^{(1-\ell) / 2}\left(0, T ; H^{-1 / 2+\ell}(\partial \Omega)^{N}\right)
$$

and

$$
\partial_{t} u(\cdot, 0) \in W
$$

The first fact follows from (10) and

$$
u \in H^{1}\left(0, T ; H^{1}(\Omega)^{N}\right) \cap L^{2}\left(0, T ; H^{2}(\Omega)\right) \subset H^{1-\ell}\left(0, T ; H^{\ell+1 / 2}(\partial \Omega)^{N}\right)
$$

continuously. Indeed, it is not difficult to see that

$$
\begin{gathered}
A_{t} \gamma_{0} u \in L^{2}\left(0, T ; H^{\nu_{2}}(\partial \Omega)^{N^{2}}\right) \cdot H^{1-\ell}\left(0, T ; H^{\ell+1 / 2}(\partial \Omega)^{N}\right) \\
\subset L^{2}\left(0, T ; H^{1 / 2}(\partial \Omega)^{N}\right) \text { continuously }
\end{gathered}
$$

and

$$
\begin{gathered}
A_{t} \gamma^{0} u \in H^{(1-\ell) / 2}\left(0, T ; H^{(3-N)(\ell-1 / 2)}(\partial \Omega)^{N^{2}}\right) \cdot H^{1-\ell}\left(0, T ; H^{\ell+1 / 2}(\partial \Omega)^{N}\right) \\
\subset H^{(1-\ell) / 2}\left(0, T ; H^{-1 / 2+\ell}(\partial \Omega)^{N}\right) \text { continuously. }
\end{gathered}
$$

Besides,

$$
\begin{aligned}
& \left\|A_{t} \gamma_{0} u\right\|_{L^{2}\left(H^{1 / 2}\right)}^{2}+\left\|A_{t} \gamma_{0} u\right\|_{H^{(1-\ell) / 2}\left(H^{-1 / 2+\ell}\right)}^{2} \\
& \leq C\|A\|_{H^{(3-\ell) / 2}\left(H^{\nu^{2}}\right)}^{2}\left(\|u\|_{H^{1}\left(H^{1}\right)}^{2}+\|u\|_{L^{2}\left(H^{2}\right)}^{2}\right)
\end{aligned}
$$

for a positive constant $C=C(\Omega)$. 
Let us now prove (42). For this, we first realize that $\theta(0) \in H^{2}(\Omega)^{N}$ from the elliptic system

$$
\left\{\begin{array}{cl}
\Delta \theta(0)=\nabla \cdot f_{4}(0) & \text { in } \Omega, \\
\frac{\partial}{\partial n} \theta(0)=\Delta u^{0} \cdot n & \text { on } \partial \Omega,
\end{array}\right.
$$

which satisfies the compatibility condition

$$
\int_{\partial \Omega} \Delta u^{0} \cdot n \mathrm{~d} \sigma=\int_{\Omega} \nabla \cdot f_{4}(0) \mathrm{d} x=0 .
$$

Hence, $\theta(0) \in H^{2}(\Omega)^{N}$ and

$$
\|\theta(0)\|_{H^{2}(\Omega)}^{2} \leq C\left(\left\|f_{4}\right\|_{L^{\infty}\left(H^{1}\right)}^{2}+\left\|u^{0}\right\|_{H^{3} \cap W}^{2}\right) .
$$

Again from the differential equation satisfied by $u$, we find

$$
\partial_{i} \partial_{t} u(0)=\partial_{i} \Delta u^{0}-\partial_{i} \nabla \theta(0)+\partial_{i}\left(f_{4}(0)\right) \in L^{2}(\Omega)^{N},
$$

so that

$$
\left\|\partial_{i} \partial_{t} u(0)\right\|_{L^{2}(\Omega)^{N}}^{2} \leq C\left(\left\|f_{4}\right\|_{L^{\infty}\left(H^{1}\right)}^{2}+\left\|u^{0}\right\|_{H^{3} \cap W}^{2}\right)
$$

for every $i \in\{1, \ldots, N\}$. Consequently, $\partial_{t} u(0) \in H^{1}(\Omega)^{N}$ and

$$
\left\|\partial_{t} u(0)\right\|_{H^{1}}^{2} \leq C\left(\left\|f_{4}\right\|_{L^{\infty}\left(H^{1}\right)}^{2}+\left\|f_{4}\right\|_{H^{1}\left(L^{2}\right)}^{2}+\left\|u^{0}\right\|_{H^{3} \cap W}^{2}\right) .
$$

As a conclusion, $\tilde{u} \in L^{2}\left(0, T ; H^{2}(\Omega)^{N} \cap W\right) \cap H^{1}(0, T ; H)$, so it has to be the case of $\partial_{t} u$ as well since $u^{0}$ satisfies (38). Furthermore,

$$
\left\|\partial_{t} u\right\|_{L^{2}\left(H^{2} \cap W\right) \cap H^{1}\left(L^{2}\right)}^{2} \leq C(\Omega, A)\left(\left\|f_{4}\right\|_{L^{\infty}\left(H^{1}\right)}^{2}+\left\|f_{4}\right\|_{H^{1}\left(L^{2}\right)}^{2}+\left\|u^{0}\right\|_{H^{3} \cap W}^{2}\right) .
$$

From this, (39) is readily deduced.

Remark 1.3. One could keep the explicit dependence of $C(\Omega, A)$ in (39) with respect to the norms of $A$ but this will not be needed, so me omit it for the sake of simplicity.

\section{Carleman inequality for the adjoint system}

In this section, we will prove a Carleman inequality for system (11). In order to do this, some weight functions are needed:

$$
\begin{gathered}
\alpha(x, t)=\frac{\mathrm{e}^{2 \lambda\left\|\eta^{0}\right\|_{\infty}}-\mathrm{e}^{\lambda \eta^{0}(x)}}{t^{4}(T-t)^{4}}, \quad \xi(x, t)=\frac{\mathrm{e}^{\lambda \eta^{0}(x)}}{t^{4}(T-t)^{4}}, \\
\tilde{\alpha}(x, t)=\frac{\mathrm{e}^{2 \lambda\left\|\eta^{0}\right\|_{\infty}-\mathrm{e}^{-\lambda \eta^{0}(x)}}}{t^{4}(T-t)^{4}}, \quad \tilde{\xi}(x, t)=\frac{\mathrm{e}^{-\lambda \eta^{0}(x)}}{t^{4}(T-t)^{4}}, \\
\alpha^{*}(t)=\max _{x \in \bar{\Omega}} \alpha(x, t)=\alpha_{\mid \partial \Omega}(x, t), \quad \widehat{\alpha}(t)=\min _{x \in \bar{\Omega}} \alpha(x, t),
\end{gathered}
$$

with $\lambda>0$ to be chosen later on. Here, $\eta^{0} \in C^{2}(\bar{\Omega})$ verifies

$$
\eta^{0}>0 \text { in } \Omega, \quad \eta^{0} \equiv 0 \text { on } \partial \Omega, \quad\left|\nabla \eta^{0}\right|>0 \text { in } \overline{\Omega \backslash \omega^{\prime}}
$$

with $\omega^{\prime} \subset \subset \omega$ an open set. Let us remark that functions of this kind were first introduced in [10] in order to obtain Carleman inequalities for the heat system. The existence of such a function is also proved in that reference. 


\subsection{Carleman inequality for the heat system}

In this paragraph, we will deduce a Carleman inequality for a (vector valued) function $\varphi$ verifying

$$
\begin{cases}-\varphi_{t}-\nabla \cdot(D \varphi)=G \in L^{2}(Q)^{N}, \quad \nabla \cdot \varphi=0 & \text { in } Q \\ \varphi \cdot n=0, \quad(D \varphi \cdot n)_{t g}+(A(x, t) \varphi)_{t g}=0 & \text { on } \Sigma .\end{cases}
$$

It is the following:

Proposition 2.1. Let A verify (8)-(9). Then, there exist three positive constants $C, \bar{s}$ and $\bar{\lambda}$ only depending on $\Omega$ and $\omega$, such that

$$
I(s, \lambda ; \varphi) \leq C\left(s^{3} \lambda^{4} \int_{0}^{T} \int_{\omega_{0}} \mathrm{e}^{-2 s \alpha} \xi^{3}|\varphi|^{2} \mathrm{~d} x \mathrm{~d} t+\lambda \iint_{Q} \mathrm{e}^{-2 s \alpha}|G|^{2} \mathrm{~d} x \mathrm{~d} t\right)
$$

for any $\lambda \geq \bar{\lambda} \mathrm{e}^{\bar{\lambda} T\|A\|_{P}^{2}}\left(1+\|A\|_{P}^{5}\right)$, any $s \geq \mathrm{e}^{4 \lambda\left\|\eta^{0}\right\|_{\infty}} \bar{s}\left(T^{6}+T^{8}\right)$ and any $\varphi \in L_{\text {loc }}^{2}\left(0, T ; L^{2}(\Omega)\right)$ verifying (46) (recall that the space $P$ was defined in (19)). Here, we have denoted

$$
\begin{gathered}
I(s, \lambda ; \varphi)=s^{3} \lambda^{4} \iint_{Q} \mathrm{e}^{-2 s \alpha} \xi^{3}|\varphi|^{2} \mathrm{~d} x \mathrm{~d} t+s \lambda^{2} \iint_{Q} \mathrm{e}^{-2 s \alpha} \xi|\nabla \varphi|^{2} \mathrm{~d} x \mathrm{~d} t \\
+s^{-1} \iint_{Q} \mathrm{e}^{-2 s \alpha} \xi^{-1}\left(\left|\varphi_{t}\right|^{2}+|\Delta \varphi|^{2}\right) \mathrm{d} x \mathrm{~d} t
\end{gathered}
$$

for each $s, \lambda>0$ and $\omega_{0}$ is an open set verifying

$$
\omega^{\prime} \subset \subset \omega_{0} \subset \subset \omega .
$$

Proof. We first observe that, by a duality argument, one can suppose that $\varphi$ is regular enough in order to justify all the integrations by parts below (for instance, $\varphi \in C^{2}(\bar{Q})$ ).

In this proof, we follow the ideas developed in [10]. More precisely, we will set

$$
\psi=\mathrm{e}^{-s \alpha} \varphi, \quad \tilde{\psi}=\mathrm{e}^{-s \tilde{\alpha}} \varphi
$$

and we will make several computations to deduce the desired inequality. To this end, let us split the proof in three steps: in the first step, we will obtain a Carleman inequality for $\psi$, in the second one a similar inequality will be deduced for $\tilde{\psi}$, while some simplifications and the conclusion will be given in the last step.

Step 1. A Carleman inequality for $\psi$.

From the equation verified by $\varphi$ we find, after some computations:

$$
M_{1} \psi+M_{2} \psi=G_{s, \lambda},
$$

with

$$
\begin{gathered}
M_{1} \psi=-\nabla \cdot\left(\nabla \psi+\nabla^{t} \psi\right)-s^{2} \lambda^{2}\left|\nabla \eta^{0}\right|^{2} \xi^{2} \psi-s \lambda \xi \nabla^{t} \psi \cdot \nabla \eta^{0}-s \alpha_{t} \psi, \\
M_{2} \psi=-\psi_{t}+2 s \lambda \xi \nabla \psi \cdot \nabla \eta^{0}+2 s \lambda \xi \nabla^{t} \psi \cdot \nabla \eta^{0}+2 s \lambda^{2}\left|\nabla \eta^{0}\right|^{2} \xi \psi
\end{gathered}
$$

and

This gives

$$
G_{s, \lambda}=\mathrm{e}^{-s \alpha} G-s \lambda \Delta \eta^{0} \xi \psi-s \lambda \xi \nabla \nabla \eta^{0} \cdot \psi+s \lambda^{2}\left|\nabla \eta^{0}\right|^{2} \xi \psi-s \lambda^{2} \nabla \eta^{0} \xi \nabla \eta^{0} \cdot \psi
$$

$$
\left\|M_{1} \psi\right\|_{L^{2}(Q)^{N}}^{2}+\left\|M_{2} \psi\right\|_{L^{2}(Q)^{N}}^{2}+2\left(M_{1} \psi, M_{2} \psi\right)_{L^{2}(Q)^{N}}=\left\|G_{s, \lambda}\right\|_{L^{2}(Q)^{N}}^{2} .
$$


Let us first develop the double product term. Then, we will conveniently get profit of the positiveness of $\left\|M_{1} \psi\right\|_{L^{2}(Q)^{N}}^{2}$ and $\left\|M_{2} \psi\right\|_{L^{2}(Q)^{N}}^{2}$.

First, we have

$$
\begin{aligned}
\left(\left(M_{1} \psi\right)_{1},\left(M_{2} \psi\right)_{1}\right)_{L^{2}(Q)^{N}}=\iint_{Q} & \left(\nabla \cdot\left(\nabla \psi+\nabla^{t} \psi\right)\right) \cdot \psi_{t} \mathrm{~d} x \mathrm{~d} t \\
\quad & =\iint_{\Sigma}\left(\left(\nabla \psi+\nabla^{t} \psi\right) \cdot n\right)_{t g} \cdot \psi_{t} \mathrm{~d} \sigma \mathrm{d} t-\iint_{Q}\left(\nabla \psi+\nabla^{t} \psi\right): \nabla \psi_{t} \mathrm{~d} x \mathrm{~d} t=A_{1}+A_{2},
\end{aligned}
$$

where

$$
A_{2}=-\frac{1}{2} \iint_{Q} \frac{\mathrm{d}}{\mathrm{d} t}\left(|\nabla \psi|^{2}+\nabla \psi: \nabla^{t} \psi\right) \mathrm{d} x \mathrm{~d} t=0 .
$$

Here, we have used the fact that $\psi \cdot n=0$ and the exponential decay of $\psi$ (and its derivatives) close to $t=0$ and $t=T$.

In addition,

$$
\begin{aligned}
\left(\left(M_{1} \psi\right)_{1},\left(M_{2} \psi\right)_{2}\right)_{L^{2}(Q)^{N}}= & -2 s \lambda \iint_{Q} \xi\left(\nabla \cdot\left(\nabla \psi+\nabla^{t} \psi\right)\right) \cdot\left(\nabla \psi \cdot \nabla \eta^{0}\right) \mathrm{d} x \mathrm{~d} t \\
= & -2 s \lambda \iint_{\Sigma} \xi \frac{\partial \eta^{0}}{\partial n}\left(\left(\nabla \psi+\nabla^{t} \psi\right) \cdot n\right) \cdot(\nabla \psi \cdot n) \mathrm{d} \sigma \mathrm{d} t \\
& +2 s \lambda \iint_{Q} \xi\left(\nabla \nabla \eta^{0} \cdot \nabla \psi\right):\left(\nabla \psi+\nabla^{t} \psi\right) \mathrm{d} x \mathrm{~d} t \\
& +2 s \lambda^{2} \iint_{Q} \xi\left(\left(\nabla \psi+\nabla^{t} \psi\right) \cdot \nabla \eta^{0}\right) \cdot\left(\nabla \psi \cdot \nabla \eta^{0}\right) \mathrm{d} x \mathrm{~d} t \\
& +2 s \lambda \iint_{Q} \xi\left(\nabla \psi+\nabla^{t} \psi\right):\left(\nabla \nabla \psi \cdot \nabla \eta^{0}\right) \mathrm{d} x \mathrm{~d} t=B+\mathcal{C}+D+E,
\end{aligned}
$$

where the properties of $\eta^{0}$ and, more precisely,

$$
\nabla \eta^{0}=\frac{\partial \eta^{0}}{\partial n} n
$$

have been employed (see (45)). Let us now compute the terms $D$ and $E$ :

$$
D=2 s \lambda^{2} \iint_{Q} \xi\left|\nabla \psi \cdot \nabla \eta^{0}\right|^{2} \mathrm{~d} x \mathrm{~d} t+2 s \lambda^{2} \iint_{Q} \xi\left(\nabla^{t} \psi \cdot \nabla \eta^{0}\right) \cdot\left(\nabla \psi \cdot \nabla \eta^{0}\right) \mathrm{d} x \mathrm{~d} t=D_{1}+D_{2} .
$$

Two integration by parts in $x$ give the following for $D_{2}$ :

$$
\begin{aligned}
D_{2} & =-2 s \lambda^{2} \iint_{Q}\left(\nabla^{t}\left(\nabla \eta^{0} \nabla \eta^{0} \xi\right) \cdot \nabla \psi\right) \cdot \psi \mathrm{d} x \mathrm{~d} t-2 s^{2} \lambda^{3} \iint_{Q} \xi \nabla \eta^{0} \cdot \nabla\left(\xi \nabla \eta^{0} \cdot \psi\right) \nabla \eta^{0} \cdot \psi \mathrm{d} x \mathrm{~d} t \\
& =-2 s \lambda^{2} \iint_{Q}\left(\nabla^{t}\left(\nabla \eta^{0} \nabla \eta^{0} \xi\right) \cdot \nabla \psi\right) \cdot \psi \mathrm{d} x \mathrm{~d} t+s^{2} \lambda^{3} \iint_{Q} \Delta \eta^{0}\left|\xi \nabla \eta^{0} \cdot \psi\right|^{2} \mathrm{~d} x \mathrm{~d} t=D_{21}+D_{22} .
\end{aligned}
$$

For $E$, we have:

$$
E=s \lambda \iint_{Q} \xi \nabla \eta^{0} \cdot \nabla|\nabla \psi|^{2} \mathrm{~d} x \mathrm{~d} t+2 s \lambda \iint_{Q} \xi \nabla^{t} \psi:\left(\nabla \nabla \psi \cdot \nabla \eta^{0}\right) \mathrm{d} x \mathrm{~d} t=E_{1}+E_{2} .
$$


Integrations by parts yield:

$E_{1}=s \lambda \iint_{\Sigma} \xi \frac{\partial \eta^{0}}{\partial n}|\nabla \psi|^{2} \mathrm{~d} \sigma \mathrm{d} t-s \lambda \iint_{Q} \Delta \eta^{0} \xi|\nabla \psi|^{2} \mathrm{~d} x \mathrm{~d} t-s \lambda^{2} \iint_{Q}\left|\nabla \eta^{0}\right|^{2} \xi|\nabla \psi|^{2} \mathrm{~d} x \mathrm{~d} t=E_{11}+E_{12}+E_{13}$ and

$$
\begin{aligned}
E_{2}= & 2 s \lambda \iint_{\Sigma} \xi \frac{\partial \eta^{0}}{\partial n} \nabla \psi: \nabla^{t} \psi \mathrm{d} \sigma \mathrm{d} t-2 s \lambda \iint_{Q} \Delta \eta^{0} \xi \nabla \psi: \nabla^{t} \psi \mathrm{d} x \mathrm{~d} t \\
& -2 s \lambda^{2} \iint_{Q}\left|\nabla \eta^{0}\right|^{2} \xi \nabla \psi: \nabla^{t} \psi \mathrm{d} x \mathrm{~d} t-2 s \lambda \iint_{Q} \xi \nabla \eta^{0} \cdot\left(\nabla \nabla^{t} \psi: \nabla \psi\right) \mathrm{d} x \mathrm{~d} t \\
= & E_{21}+E_{22}+E_{23}+E_{24} .
\end{aligned}
$$

Let us deal again with the last term:

$$
\begin{aligned}
E_{24}= & -2 s \lambda \iint_{\Sigma} \xi \frac{\partial \eta^{0}}{\partial n}(\nabla \psi \cdot n) \cdot\left(\nabla^{t} \psi \cdot n\right) \mathrm{d} \sigma \mathrm{d} t \\
& +2 s \lambda \iint_{Q} \xi\left(\nabla \nabla \eta^{0} \cdot \nabla^{t} \psi\right) \cdot \nabla \psi \mathrm{d} x \mathrm{~d} t+2 s \lambda^{2} \iint_{Q} \xi\left(\nabla^{t} \psi \cdot \nabla \eta^{0}\right) \cdot\left(\nabla \psi \cdot \nabla \eta^{0}\right) \mathrm{d} x \mathrm{~d} t \\
& +2 s^{2} \lambda^{2} \iint_{Q} \xi^{2}\left(\nabla \nabla \eta^{0} \cdot \psi\right) \cdot\left(\nabla \psi \cdot \nabla \eta^{0}\right) \mathrm{d} x \mathrm{~d} t \\
& +s^{2} \lambda^{3} \iint_{Q} \xi^{2} \nabla \eta^{0} \cdot\left(\nabla(\psi \psi) \cdot \nabla \eta^{0} \cdot \nabla \eta^{0}\right) \mathrm{d} x \mathrm{~d} t \\
& +2 s^{2} \lambda^{2} \iint_{Q} \xi^{2}\left(\nabla \psi \cdot \nabla \eta^{0}\right) \cdot\left(\nabla^{t} \psi \cdot \nabla \eta^{0}\right) \mathrm{d} x \mathrm{~d} t \\
= & E_{241}+E_{242}+D_{2}+E_{243}+E_{244}+E_{245} \cdot
\end{aligned}
$$

Here, we have used the fact that $\nabla \cdot \psi=s \lambda \xi \nabla \eta^{0} \cdot \psi$. Finally, since $\eta^{0} \in C^{2}(\bar{\Omega})$, we obtain

$$
E_{244} \geq-C(1+\lambda) s^{2} \lambda^{3} \iint_{Q} \xi^{2}|\psi|^{2} \mathrm{~d} x \mathrm{~d} t
$$

and

$$
\begin{aligned}
E_{245}= & -2 s^{2} \lambda^{2} \iint_{Q} \xi^{2}\left(\nabla\left(\nabla \eta^{0} \nabla \eta^{0}\right) \cdot \nabla^{t} \psi\right) \cdot \psi \mathrm{d} x \mathrm{~d} t-2 E_{244} \\
& -s^{3} \lambda^{3} \iint_{Q} \xi \nabla \eta^{0} \cdot \nabla\left|\xi \psi \cdot \nabla \eta^{0}\right|^{2} \mathrm{~d} x \mathrm{~d} t \\
= & -2 s^{2} \lambda^{2} \iint_{Q} \xi^{2}\left(\nabla\left(\nabla \eta^{0} \nabla \eta^{0}\right) \cdot \nabla^{t} \psi\right) \cdot \psi \mathrm{d} x \mathrm{~d} t-2 E_{244} \\
& +s^{3} \lambda^{3} \iint_{Q} \Delta \eta^{0} \xi^{3}\left|\psi \cdot \nabla \eta^{0}\right|^{2} \mathrm{~d} x \mathrm{~d} t+s^{3} \lambda^{4} \iint_{Q}\left|\nabla \eta^{0}\right|^{2} \xi^{3}\left|\psi \cdot \nabla \eta^{0}\right|^{2} \mathrm{~d} x \mathrm{~d} t \\
= & E_{2451}-2 E_{244}+E_{2452}+E_{2453} .
\end{aligned}
$$


We also have

$$
\begin{aligned}
\left(\left(M_{1} \psi\right)_{1},\right. & \left.\left(M_{2} \psi\right)_{3}\right)_{L^{2}(Q)^{N}}=-2 s \lambda \iint_{Q} \xi \nabla \cdot\left(\nabla \psi+\nabla^{t} \psi\right) \cdot\left(\nabla^{t} \psi \cdot \nabla \eta^{0}\right) \mathrm{d} x \mathrm{~d} t \\
= & -2 s \lambda \iint_{\Sigma} \xi \frac{\partial \eta^{0}}{\partial n}\left(\left(\nabla \psi+\nabla^{t} \psi\right) \cdot n\right) \cdot\left(\nabla^{t} \psi \cdot n\right) \mathrm{d} \sigma \mathrm{d} t \\
& +2 s \lambda \iint_{Q} \xi\left(\nabla \psi+\nabla^{t} \psi\right):\left(\nabla \nabla \eta^{0} \cdot \nabla^{t} \psi\right) \mathrm{d} x \mathrm{~d} t \\
& +2 s \lambda^{2} \iint_{Q} \xi\left(\nabla \psi \cdot \nabla \eta^{0}\right) \cdot\left(\nabla^{t} \psi \cdot \nabla \eta^{0}\right) \mathrm{d} x \mathrm{~d} t+2 s \lambda^{2} \iint_{Q} \xi\left|\nabla^{t} \psi \cdot \nabla \eta^{0}\right|^{2} \mathrm{~d} x \mathrm{~d} t \\
& +4 s \lambda \iint_{Q} \xi\left(\nabla \psi:\left(\nabla \eta^{0} \cdot \nabla^{t} \nabla \psi\right)\right) \mathrm{d} x \mathrm{~d} t \\
= & F_{1}+F_{2}+G_{1}+G_{2}+H .
\end{aligned}
$$

Using $\psi \cdot n=0$ and the expression of $\nabla \cdot \psi$, we obtain the following for $G_{1}$ :

$$
\begin{aligned}
G_{1}= & -2 s \lambda^{2} \iint_{Q} \xi\left(\nabla \nabla \eta^{0}: \nabla \psi\right)\left(\psi \cdot \nabla \eta^{0}\right) \mathrm{d} x \mathrm{~d} t \\
& -2 s \lambda^{3} \iint_{Q} \xi\left(\left(\nabla \psi \cdot \nabla \eta^{0}\right) \cdot \nabla \eta^{0}\right)\left(\psi \cdot \nabla \eta^{0}\right) \mathrm{d} x \mathrm{~d} t-s^{2} \lambda^{3} \iint_{Q} \nabla \eta^{0} \cdot \nabla\left|\xi \psi \cdot \nabla \eta^{0}\right|^{2} \mathrm{~d} x \mathrm{~d} t \\
= & G_{11}+G_{12}+D_{22} .
\end{aligned}
$$

On the other hand,

$$
\begin{aligned}
H= & 4 s \lambda \iint_{\Sigma} \xi \frac{\partial \eta^{0}}{\partial n}\left|\nabla^{t} \psi \cdot n\right|^{2} \mathrm{~d} \sigma \mathrm{d} t-4 s \lambda \iint_{Q} \xi\left(\nabla \nabla \eta^{0} \cdot \nabla^{t} \psi\right) \cdot \nabla^{t} \psi \mathrm{d} x \mathrm{~d} t \\
& -4 s \lambda^{2} \iint_{Q} \xi\left|\nabla^{t} \psi \cdot \nabla \eta^{0}\right|^{2} \mathrm{~d} x \mathrm{~d} t-4 s^{2} \lambda^{2} \iint_{Q} \xi \nabla\left(\xi \psi \cdot \nabla \eta^{0}\right) \cdot\left(\nabla^{t} \psi \cdot \nabla \eta^{0}\right) \mathrm{d} x \mathrm{~d} t \\
= & H_{1}+H_{2}+H_{3}+H_{4} .
\end{aligned}
$$

Let us then rewrite the fourth term like this:

$$
\begin{aligned}
H_{4}= & -4 s^{2} \lambda^{2} \iint_{Q} \xi^{2}\left(\nabla \nabla \eta^{0} \cdot \psi\right) \cdot\left(\nabla^{t} \psi \cdot \nabla \eta^{0}\right) \mathrm{d} x \mathrm{~d} t \\
& -4 s^{2} \lambda^{3} \iint_{Q} \xi^{2}\left(\psi \cdot \nabla \eta^{0}\right) \nabla \eta^{0} \cdot\left(\nabla^{t} \psi \cdot \nabla \eta^{0}\right) \mathrm{d} x \mathrm{~d} t-4 s^{2} \lambda^{2} \iint_{Q} \xi^{2}\left|\nabla^{t} \psi \cdot \nabla \eta^{0}\right|^{2} \mathrm{~d} x \mathrm{~d} t \\
= & H_{41}-2 E_{244}+H_{42} .
\end{aligned}
$$


To finish with the double products of the first term in $M_{1} \psi$, let us compute the following:

$$
\begin{aligned}
\left(\left(M_{1} \psi\right)_{1},\left(M_{2} \psi\right)_{4}\right)_{L^{2}(Q)^{N}}= & -2 s \lambda^{2} \iint_{Q}\left|\nabla \eta^{0}\right|^{2} \xi\left(\nabla \cdot\left(\nabla \psi+\nabla^{t} \psi\right)\right) \cdot \psi \mathrm{d} x \mathrm{~d} t \\
= & -2 s \lambda^{2} \iint_{\Sigma} \xi\left|\frac{\partial \eta^{0}}{\partial n}\right|^{2}\left(\left(\nabla \psi+\nabla^{t} \psi\right) \cdot n\right)_{t g} \cdot \psi \mathrm{d} \sigma \mathrm{d} t \\
& +4 s \lambda^{2} \iint_{Q} \xi\left(\nabla \nabla \eta^{0} \nabla \eta^{0}\right) \cdot\left(\left(\nabla \psi+\nabla^{t} \psi\right) \cdot \psi\right) \mathrm{d} x \mathrm{~d} t \\
& +2 s \lambda^{3} \iint_{Q}\left|\nabla \eta^{0}\right|^{2} \xi\left(\nabla \eta^{0} \cdot\left(\nabla \psi+\nabla^{t} \psi\right)\right) \cdot \psi \mathrm{d} x \mathrm{~d} t \\
& +2 s \lambda^{2} \iint_{Q}\left|\nabla \eta^{0}\right|^{2} \xi|\nabla \psi|^{2} \mathrm{~d} x \mathrm{~d} t+2 s \lambda^{2} \iint_{Q}\left|\nabla \eta^{0}\right|^{2} \xi \nabla \psi: \nabla^{t} \psi \mathrm{d} x \mathrm{~d} t \\
=I_{1}+I_{2}+I_{3}+I_{4}+I_{5} . &
\end{aligned}
$$

Consequently, we obtain

$$
\begin{aligned}
& \left(\left(M_{1} \psi\right)_{1}, M_{2} \psi\right)_{L^{2}(Q)^{N}}= \\
& \left(\left(M_{1} \psi\right)_{1},\left(M_{2} \psi\right)_{1}+\left(M_{2} \psi\right)_{2}+\left(M_{2} \psi\right)_{3}+\left(M_{2} \psi\right)_{4}\right)_{L^{2}(Q)^{N}} \\
& =A_{1}+B+\mathcal{C}+D_{1}+2 D_{21}+3 D_{22}+E_{11}+E_{12}+E_{13}+E_{21}+E_{22} \\
& +E_{23}+E_{241}+E_{242}+E_{243}-3 E_{244}+E_{2452}+E_{2453}+F_{1}+F_{2}+G_{11} \\
& +G_{12}+H_{1}+H_{2}+H_{3}+H_{41}+H_{42}+I_{1}+I_{2}+I_{3}+I_{4}+I_{5} .
\end{aligned}
$$

Watching carefully the expressions of these integrals, we observe that

$$
\begin{aligned}
& \mathcal{C}, E_{12}, E_{22}, E_{242}, F_{2}, H_{2} \leq C s \lambda \iint_{Q} \xi|\nabla \psi|^{2} \mathrm{~d} x \mathrm{~d} t \\
& D_{21}, F_{2}, G_{11}, I_{2} \leq C\left(s \lambda \iint_{Q} \xi|\nabla \psi|^{2} \mathrm{~d} x \mathrm{~d} t+s \lambda^{3} \iint_{Q} \xi|\psi|^{2} \mathrm{~d} x \mathrm{~d} t\right) \\
& D_{22} \leq C s^{2} \lambda^{3} \iint_{Q} \xi^{2}|\psi|^{2} \mathrm{~d} x \mathrm{~d} t \\
& E_{13}+I_{4}=s \lambda^{2} \iint_{Q}\left|\nabla \eta^{0}\right|^{2} \xi|\nabla \psi|^{2} \mathrm{~d} x \mathrm{~d} t \\
& E_{243}, H_{41} \leq C\left(s \lambda \iint_{Q} \xi|\nabla \psi|^{2} \mathrm{~d} x \mathrm{~d} t+s^{3} \lambda^{3} \iint_{Q} \xi^{3}|\psi|^{2} \mathrm{~d} x \mathrm{~d} t\right) \\
& E_{2452} \leq C s^{3} \lambda^{3} \iint_{Q} \xi^{3}|\psi|^{2} \mathrm{~d} x \mathrm{~d} t \\
& G_{12}, I_{3} \leq C\left(\lambda^{2} \iint_{Q} \xi|\nabla \psi|^{2} \mathrm{~d} x \mathrm{~d} t+s^{2} \lambda^{4} \iint_{Q} \xi|\psi|^{2} \mathrm{~d} x \mathrm{~d} t\right) \\
& G_{2}+H_{3}=-2 s \lambda^{2} \iint_{Q} \xi\left|\nabla^{t} \psi \cdot \nabla \eta^{0}\right|^{2} \mathrm{~d} x \mathrm{~d} t
\end{aligned}
$$


and $D_{1} \geq 0, E_{23}+I_{4}=0$. All this, together with the estimate of $E_{244}$, provides from (51):

$$
\begin{aligned}
& \left(\left(M_{1} \psi\right)_{1}, M_{2} \psi\right)_{L^{2}(Q)^{N}} \geq \iint_{\Sigma}\left(\left(\nabla \psi+\nabla^{t} \psi\right) \cdot n\right)_{t g} \cdot \psi_{t} \mathrm{~d} \sigma \mathrm{d} t \\
& -2 s \lambda \iint_{\Sigma} \xi \frac{\partial \eta^{0}}{\partial n}\left(\left(\nabla \psi+\nabla^{t} \psi\right) \cdot n\right) \cdot(\nabla \psi \cdot n) \mathrm{d} \sigma \mathrm{d} t \\
& +s \lambda \iint_{\Sigma} \xi \frac{\partial \eta^{0}}{\partial n}|\nabla \psi|^{2} \mathrm{~d} \sigma \mathrm{d} t+2 s \lambda \iint_{\Sigma} \xi \frac{\partial \eta^{0}}{\partial n} \nabla \psi: \nabla^{t} \psi \mathrm{d} \sigma \mathrm{d} t \\
& -2 s \lambda \iint_{\Sigma} \xi \frac{\partial \eta^{0}}{\partial n}(\nabla \psi \cdot n) \cdot\left(\nabla^{t} \psi \cdot n\right) \mathrm{d} \sigma \mathrm{d} t \\
& -2 s \lambda \iint_{\Sigma} \xi \frac{\partial \eta^{0}}{\partial n}\left(\left(\nabla \psi+\nabla^{t} \psi\right) \cdot n\right) \cdot\left(\nabla^{t} \psi \cdot n\right) \mathrm{d} \sigma \mathrm{d} t \\
& +4 s \lambda \iint_{\Sigma} \xi \frac{\partial \eta^{0}}{\partial n}\left|\nabla^{t} \psi \cdot n\right|^{2} \mathrm{~d} \sigma \mathrm{d} t \\
& -2 s \lambda^{2} \iint_{\Sigma} \xi\left|\frac{\partial \eta^{0}}{\partial n}\right|^{2}\left(\left(\nabla \psi+\nabla^{t} \psi\right) \cdot n\right)_{t g} \cdot \psi \mathrm{d} \sigma \mathrm{d} t \\
& +s \lambda^{2} \iint_{Q}\left|\nabla \eta^{0}\right|^{2} \xi|\nabla \psi|^{2} \mathrm{~d} x \mathrm{~d} t+s^{3} \lambda^{4} \iint_{Q}\left|\nabla \eta^{0}\right|^{2} \xi^{3}\left|\psi \cdot \nabla \eta^{0}\right|^{2} \mathrm{~d} x \mathrm{~d} t \\
& -2 s \lambda^{2} \iint_{Q} \xi\left|\nabla^{t} \psi \cdot \nabla \eta^{0}\right|^{2} \mathrm{~d} x \mathrm{~d} t-4 s^{2} \lambda^{2} \iint_{Q} \xi^{2}\left|\nabla^{t} \psi \cdot \nabla \eta^{0}\right|^{2} \mathrm{~d} x \mathrm{~d} t \\
& -C\left(s \lambda \iint_{Q} \xi|\nabla \psi|^{2} \mathrm{~d} x \mathrm{~d} t+s \lambda^{3}\left(s+s^{2}+\lambda\right) \iint_{Q} \xi^{3}|\psi|^{2} \mathrm{~d} x \mathrm{~d} t\right) .
\end{aligned}
$$

On the other hand, we have

$$
\begin{aligned}
\left(\left(M_{1} \psi\right)_{2},\left(M_{2} \psi\right)_{1}\right)_{L^{2}(Q)^{N}} & =\frac{1}{2} s^{2} \lambda^{2} \iint_{Q}\left|\nabla \eta^{0}\right|^{2} \xi^{2} \frac{\partial}{\partial t}|\psi|^{2} \mathrm{~d} x \mathrm{~d} t \\
& =-s^{2} \lambda^{2} \iint_{Q}\left|\nabla \eta^{0}\right|^{2} \xi \xi_{t}|\psi|^{2} \mathrm{~d} x \mathrm{~d} t=J
\end{aligned}
$$

In addition, we find that

$$
\begin{aligned}
&\left(\left(M_{1} \psi\right)_{2},\left(M_{2} \psi\right)_{2}\right)_{L^{2}(Q)^{N}}=-s^{2} \lambda^{3} \iint_{Q}\left|\nabla \eta^{0}\right|^{2} \xi^{3} \nabla \eta^{0} \cdot \nabla|\psi|^{2} \mathrm{~d} x \mathrm{~d} t \\
&=-s^{3} \lambda^{3} \iint_{\Sigma} \xi^{3}\left(\frac{\partial \eta^{0}}{\partial n}\right)^{3}|\psi|^{2} \mathrm{~d} \sigma \mathrm{d} t+s^{3} \lambda^{3} \iint_{Q} \xi^{3} \nabla \cdot\left(\left|\nabla \eta^{0}\right|^{2} \nabla \eta^{0}\right)|\psi|^{2} \mathrm{~d} x \mathrm{~d} t \\
&+3 s^{3} \lambda^{4} \iint_{Q}\left|\nabla \eta^{0}\right|^{4} \xi^{3}|\psi|^{2} \mathrm{~d} x \mathrm{~d} t=K_{1}+K_{2}+K_{3} .
\end{aligned}
$$

Let us know skip the product

$$
\left(\left(M_{1} \psi\right)_{2},\left(M_{2} \psi\right)_{3}\right)_{L^{2}(Q)^{N}}
$$

In fact, a computation of this term will not be crucial for the sequel, since it will be compensated with another one. More details will be given below. 
The last product for $\left(M_{1} \psi\right)_{2}$ gives:

$$
\left(\left(M_{1} \psi\right)_{2},\left(M_{2} \psi\right)_{4}\right)_{L^{2}(Q)^{N}}=-2 s^{3} \lambda^{4} \iint_{Q}\left|\nabla \eta^{0}\right|^{4} \xi^{3}|\psi|^{2} \mathrm{~d} x \mathrm{~d} t=L
$$

Consequently, we deduce that

$$
\begin{aligned}
\left(\left(M_{1} \psi\right)_{2}, M_{2} \psi\right)_{L^{2}(Q)^{N}} & =\left(\left(M_{1} \psi\right)_{2},\left(M_{2} \psi\right)_{1}+\left(M_{2} \psi\right)_{2}+\left(M_{2} \psi\right)_{3}+\left(M_{2} \psi\right)_{4}\right)_{L^{2}(Q)} \\
& =J+K_{1}+K_{2}+K_{3}+L+\left(\left(M_{1} \psi\right)_{2},\left(M_{2} \psi\right)_{3}\right)_{L^{2}(Q)^{N}}
\end{aligned}
$$

Taking into account that $\eta^{0}>0$ in $\Omega$, we find that

$$
\xi_{t} \leq C T \xi^{5 / 4}
$$

and so

$$
\begin{aligned}
\left(\left(M_{1} \psi\right)_{2}, M_{2} \psi\right)_{L^{2}(Q)^{N}} \geq & -s^{3} \lambda^{3} \iint_{\Sigma} \xi^{3}\left(\frac{\partial \eta^{0}}{\partial n}\right)^{3}|\psi|^{2} \mathrm{~d} \sigma \mathrm{d} t \\
& +s^{3} \lambda^{4} \iint_{Q}\left|\nabla \eta^{0}\right|^{4} \xi^{3}|\psi|^{2} \mathrm{~d} x \mathrm{~d} t+\left(\left(M_{1} \psi\right)_{2},\left(M_{2} \psi\right)_{3}\right)_{L^{2}(Q)^{N}} \\
& -C\left(s^{2} \lambda^{2} T \iint_{Q} \xi^{9 / 4}|\psi|^{2} \mathrm{~d} x \mathrm{~d} t+s^{3} \lambda^{3} \iint_{Q} \xi^{3}|\psi|^{2} \mathrm{~d} x \mathrm{~d} t\right) .
\end{aligned}
$$

Let us now consider the scalar products of the third term in $M_{1} \psi$. First of all, we have:

$$
\begin{aligned}
& \left(\left(M_{1} \psi\right)_{3},\left(M_{2} \psi\right)_{1}\right)_{L^{2}(Q)^{N}}=s \lambda \iint_{Q} \xi\left(\nabla^{t} \psi \cdot \nabla \eta^{0}\right) \cdot \psi_{t} \mathrm{~d} x \mathrm{~d} t \\
& \quad=-s \lambda \iint_{Q} \xi\left(\nabla \nabla \eta^{0} \cdot \psi\right) \cdot \psi_{t} \mathrm{~d} x \mathrm{~d} t-s \lambda^{2} \iint_{Q} \xi\left(\psi \cdot \nabla \eta^{0}\right)\left(\psi_{t} \cdot \nabla \eta^{0}\right) \mathrm{d} x \mathrm{~d} t \\
& \quad-s^{2} \lambda^{2} \iint_{Q} \xi\left(\psi \cdot \nabla \eta^{0}\right) \nabla \eta^{0} \cdot(\xi \psi)_{t} \mathrm{~d} x \mathrm{~d} t=M_{1}+M_{2}+M_{3}
\end{aligned}
$$

Here, we must remark that, due to the exponential decay of $\psi$ at $t=0$ and $t=T, M_{3}=0$, while

$$
M_{1}=\frac{1}{2} s \lambda \iint_{Q} \xi_{t}\left(\nabla \nabla \eta^{0} \cdot \psi\right) \cdot \psi \mathrm{d} x \mathrm{~d} t
$$

and

$$
M_{2}=\frac{1}{2} s \lambda^{2} \iint_{Q} \xi_{t}\left|\psi \cdot \nabla \eta^{0}\right|^{2} \mathrm{~d} x \mathrm{~d} t
$$


Furthermore,

$$
\begin{aligned}
\left(\left(M_{1} \psi\right)_{3},\right. & \left.\left(M_{2} \psi\right)_{2}\right)_{L^{2}(Q)^{N}}=-2 s^{2} \lambda^{2} \iint_{Q} \xi^{2}\left(\nabla^{t} \psi \cdot \nabla \eta^{0}\right) \cdot\left(\nabla \psi \cdot \nabla \eta^{0}\right) \mathrm{d} x \mathrm{~d} t \\
& =2 s^{2} \lambda^{2} \iint_{Q} \xi^{2}\left(\nabla^{t} \psi \cdot \nabla\left(\nabla \eta^{0} \nabla \eta^{0}\right)\right) \cdot \psi \mathrm{d} x \mathrm{~d} t \\
& +4 s^{2} \lambda^{3} \iint_{Q} \xi^{2}\left(\psi \cdot \nabla \eta^{0}\right)\left(\nabla \psi \cdot \nabla \eta^{0}\right) \cdot \nabla \eta^{0} \mathrm{~d} x \mathrm{~d} t \\
& +2 s^{3} \lambda^{3} \iint_{Q} \xi^{2}\left(\psi \cdot \nabla \eta^{0}\right) \nabla \eta^{0} \cdot \nabla\left(\xi \psi \cdot \nabla \eta^{0}\right) \mathrm{d} x \mathrm{~d} t=N_{1}+2 E_{244}+N_{2}
\end{aligned}
$$

Let us compute the last integral:

$$
\begin{aligned}
N_{2} & =s^{3} \lambda^{3} \iint_{Q} \xi \nabla \eta^{0} \cdot \nabla\left|\xi \psi \cdot \nabla \eta^{0}\right|^{2} \mathrm{~d} x \mathrm{~d} t \\
& =-s^{3} \lambda^{3} \iint_{Q} \xi \Delta \eta^{0}\left|\xi \psi \cdot \nabla \eta^{0}\right|^{2} \mathrm{~d} x \mathrm{~d} t-s^{3} \lambda^{4} \iint_{Q}\left|\nabla \eta^{0}\right|^{2} \xi^{3}\left|\psi \cdot \nabla \eta^{0}\right|^{2} \mathrm{~d} x \mathrm{~d} t \\
& =N_{21}+N_{22} .
\end{aligned}
$$

Furthermore, we have

$$
\left(\left(M_{1} \psi\right)_{3},\left(M_{2} \psi\right)_{3}\right)_{L^{2}(Q)^{N}}=-2 s^{2} \lambda^{2} \iint_{Q}\left|\nabla \eta^{0}\right|^{2} \xi^{2}\left|\nabla^{t} \psi \cdot \nabla \eta^{0}\right|^{2} \mathrm{~d} x \mathrm{~d} t=O
$$

The last product for $\left(M_{1} \psi\right)_{3}$ gives

$$
\begin{aligned}
\left(\left(M_{1} \psi\right)_{3},\left(M_{2} \psi\right)_{4}\right)_{L^{2}(Q)^{N}}= & -2 s^{2} \lambda^{3} \iint_{Q}\left|\nabla \eta^{0}\right|^{2} \xi^{2}\left(\nabla^{t} \psi \cdot \nabla \eta^{0}\right) \cdot \psi \mathrm{d} x \mathrm{~d} t \\
= & 2 s^{2} \lambda^{3} \iint_{Q}\left(\nabla\left(\left|\nabla \eta^{0}\right|^{2} \xi^{2} \nabla \eta^{0}\right) \cdot \psi\right) \cdot \psi \mathrm{d} x \mathrm{~d} t \\
& +2 s^{3} \lambda^{4} \iint_{Q}\left|\nabla \eta^{0}\right|^{2} \xi^{3}\left|\psi \cdot \nabla \eta^{0}\right|^{2} \mathrm{~d} x \mathrm{~d} t=P_{1}+P_{2}
\end{aligned}
$$

We will use here, as we did before, the following estimate for $P_{1}$ :

$$
P_{1} \leq C s^{2} \lambda^{3}(1+\lambda) \iint_{Q} \xi^{2}|\psi|^{2} \mathrm{~d} x \mathrm{~d} t
$$

Putting all the products of $\left(M_{1} \psi\right)_{3}$ together, we find

$$
\begin{aligned}
\left(\left(M_{1} \psi\right)_{3}, M_{2} \psi\right)_{L^{2}(Q)^{N}} & =\left(\left(M_{1} \psi\right)_{3},\left(M_{2} \psi\right)_{1}+\left(M_{2} \psi\right)_{2}+\left(M_{2} \psi\right)_{3}+\left(M_{2} \psi\right)_{4}\right)_{L^{2}(Q)^{N}} \\
& =M_{1}+M_{2}+N_{1}+2 E_{244}+N_{21}+N_{22}+O+P_{1}+P_{2} .
\end{aligned}
$$


Now, using the properties of $\eta^{0}$ and Holder's inequality, we have

$$
\begin{aligned}
\left(\left(M_{1} \psi\right)_{3}, M_{2} \psi\right)_{L^{2}(Q)^{N}} \geq & s^{3} \lambda^{4} \iint_{Q}\left|\nabla \eta^{0}\right|^{2} \xi^{3}\left|\psi \cdot \nabla \eta^{0}\right|^{2} \mathrm{~d} x \mathrm{~d} t \\
& -2 s^{2} \lambda^{2} \iint_{Q}\left|\nabla \eta^{0}\right|^{2} \xi^{2}\left|\nabla^{t} \psi \cdot \nabla \eta^{0}\right|^{2} \mathrm{~d} x \mathrm{~d} t \\
& -C\left(s \lambda T(1+\lambda) \iint_{Q} \xi^{5 / 4}|\psi|^{2} \mathrm{~d} x \mathrm{~d} t\right. \\
& +s \lambda \iint_{Q} \xi|\nabla \psi|^{2} \mathrm{~d} x \mathrm{~d} t+s^{3} \lambda^{3} \iint_{Q} \xi^{3}|\psi|^{2} \mathrm{~d} x \mathrm{~d} t \\
& \left.+s^{2} \lambda^{3}(1+\lambda) \iint_{Q} \xi^{2}|\psi|^{2} \mathrm{~d} x \mathrm{~d} t\right) .
\end{aligned}
$$

Let us finally perform the computations for the fourth and last term in the expression of $M_{1} \psi$.

First, we have:

$$
\begin{aligned}
\left(\left(M_{1} \psi\right)_{4},\left(M_{2} \psi\right)_{1}\right)_{L^{2}(Q)^{N}} & =\frac{1}{2} s \iint_{Q} \alpha_{t} \frac{\partial}{\partial t}|\psi|^{2} \mathrm{~d} x \mathrm{~d} t \\
& =-\frac{1}{2} s \iint_{Q} \alpha_{t t}|\psi|^{2} \mathrm{~d} x \mathrm{~d} t=Q
\end{aligned}
$$

Then,

$$
\begin{aligned}
& \left(\left(M_{1} \psi\right)_{4},\left(M_{2} \psi\right)_{2}\right)_{L^{2}(Q)^{N}}=-s^{2} \lambda \iint_{Q} \xi \alpha_{t} \nabla \eta^{0} \cdot|\psi|^{2} \mathrm{~d} x \mathrm{~d} t \\
& \quad=-s^{2} \lambda \iint_{\Sigma} \frac{\partial \eta^{0}}{\partial n} \xi \alpha_{t}|\psi|^{2} \mathrm{~d} \sigma \mathrm{d} t+s^{2} \lambda \iint_{Q} \nabla \cdot\left(\xi \alpha_{t} \nabla \eta^{0}\right)|\psi|^{2} \mathrm{~d} x \mathrm{~d} t=R_{1}+R_{2} .
\end{aligned}
$$

Let us again skip the term

$$
\left(\left(M_{1} \psi\right)_{4},\left(M_{2} \psi\right)_{3}\right)_{L^{2}(Q)^{N}}
$$

Finally, we have

$$
\left(\left(M_{1} \psi\right)_{4},\left(M_{2} \psi\right)_{4}\right)_{L^{2}(Q)^{N}}=-2 s^{2} \lambda^{2} \iint_{Q}\left|\nabla \eta^{0}\right|^{2} \xi \alpha_{t}|\psi|^{2} \mathrm{~d} x \mathrm{~d} t=S
$$

All the computations made for $\left(M_{1} \psi\right)_{4}$ yields

$$
\left(\left(M_{1} \psi\right)_{4}, M_{2} \psi\right)_{L^{2}(Q)^{N}}=Q+R_{1}+R_{2}+S+\left(\left(M_{1} \psi\right)_{4},\left(M_{2} \psi\right)_{3}\right)_{L^{2}(Q)^{N}}
$$

Let us then deal with the expression of $\alpha_{t}$ :

$$
\alpha_{t}=-4(T-2 t)\left(\mathrm{e}^{2 \lambda\left\|\eta^{0}\right\|_{\infty}}-\mathrm{e}^{\lambda \eta^{0}}\right)(t(T-t))^{-5}
$$

From the properties of $\eta^{0}$, we deduce

$$
\left|\alpha_{t}\right| \leq C T \mathrm{e}^{2 \lambda\left\|\eta^{0}\right\|_{\infty}} \xi^{5 / 4}
$$

On the other hand, it is not difficult to check these two other estimates:

$$
\begin{gathered}
\left|\nabla \alpha_{t}\right| \leq C \lambda T \xi^{5 / 4} \\
\left|\alpha_{t t}\right| \leq C \mathrm{e}^{2 \lambda\left\|\eta^{0}\right\|_{\infty}}\left(T^{2} \xi^{3 / 2}+\xi^{5 / 4}\right) .
\end{gathered}
$$


Consequently, we find the following for $\left(M_{1} \psi\right)_{4}$ :

$$
\begin{aligned}
& \left(\left(M_{1} \psi\right)_{4}, M_{2} \psi\right)_{L^{2}(Q)^{N}} \geq-s^{2} \lambda \iint_{\Sigma} \frac{\partial \eta^{0}}{\partial n} \xi \alpha_{t}|\psi|^{2} \mathrm{~d} \sigma \mathrm{d} t \\
& +\left(\left(M_{1} \psi\right)_{4},\left(M_{2} \psi\right)_{3}\right)_{L^{2}(Q)^{N}} \\
& -C \mathrm{e}^{2 \lambda\left\|\eta^{0}\right\|_{\infty}}\left(s \iint_{Q}\left(T^{2} \xi^{3 / 2}+\xi^{5 / 4}\right)|\psi|^{2} \mathrm{~d} x \mathrm{~d} t\right. \\
& \left.+s^{2} \lambda(1+\lambda) T \iint_{Q} \xi^{9 / 4}|\psi|^{2} \mathrm{~d} x \mathrm{~d} t\right)
\end{aligned}
$$

As a conclusion, taking into account (52), (53), (54), (56) and

$$
\xi^{-1} \leq C T^{8}
$$

we obtain

$$
\begin{aligned}
& 2\left(M_{1} \psi, M_{2} \psi\right)_{L^{2}(Q)^{N}} \geq 2 \iint_{\Sigma}\left(\left(\nabla \psi+\nabla^{t} \psi\right) \cdot n\right)_{t g} \cdot \psi_{t} \mathrm{~d} \sigma \mathrm{d} t \\
& -4 s \lambda \iint_{\Sigma} \xi \frac{\partial \eta^{0}}{\partial n}\left(\left(\nabla \psi+\nabla^{t} \psi\right) \cdot n\right) \cdot(\nabla \psi \cdot n) \mathrm{d} \sigma \mathrm{d} t \\
& +2 s \lambda \iint_{\Sigma} \xi \frac{\partial \eta^{0}}{\partial n}|\nabla \psi|^{2} \mathrm{~d} \sigma \mathrm{d} t+4 s \lambda \iint_{\Sigma} \xi \frac{\partial \eta^{0}}{\partial n} \nabla \psi: \nabla^{t} \psi \mathrm{d} \sigma \mathrm{d} t \\
& -4 s \lambda \iint_{\Sigma} \xi \frac{\partial \eta^{0}}{\partial n}(\nabla \psi \cdot n) \cdot\left(\nabla^{t} \psi \cdot n\right) \mathrm{d} \sigma \mathrm{d} t \\
& -4 s \lambda \iint_{\Sigma} \xi \frac{\partial \eta^{0}}{\partial n}\left(\left(\nabla \psi+\nabla^{t} \psi\right) \cdot n\right) \cdot\left(\nabla^{t} \psi \cdot n\right) \mathrm{d} \sigma \mathrm{d} t \\
& -4 s \lambda^{2} \iint_{\Sigma} \xi\left|\frac{\partial \eta^{0}}{\partial n}\right|^{2}\left(\left(\nabla \psi+\nabla^{t} \psi\right) \cdot n\right)_{t g} \cdot \psi \mathrm{d} \sigma \mathrm{d} t \\
& -2 s^{3} \lambda^{3} \iint_{\Sigma} \xi^{3}\left(\frac{\partial \eta^{0}}{\partial n}\right)^{3}|\psi|^{2} \mathrm{~d} x \mathrm{~d} t-2 s^{2} \lambda \iint_{\Sigma} \frac{\partial \eta^{0}}{\partial n} \xi \alpha_{t}|\psi|^{2} \mathrm{~d} x \mathrm{~d} t \\
& +8 s \lambda \iint_{\Sigma} \xi \frac{\partial \eta^{0}}{\partial n}\left|\nabla^{t} \psi \cdot n\right|^{2} \mathrm{~d} \sigma \mathrm{d} t+4 s^{3} \lambda^{4} \iint_{Q}\left|\nabla \eta^{0}\right|^{2} \xi^{3}\left|\psi \cdot \nabla \eta^{0}\right|^{2} \mathrm{~d} x \mathrm{~d} t \\
& +2 s \lambda^{2} \iint_{Q}\left|\nabla \eta^{0}\right|^{2} \xi|\nabla \psi|^{2} \mathrm{~d} x \mathrm{~d} t+2 s^{3} \lambda^{4} \iint_{Q}\left|\nabla \eta^{0}\right|^{4} \xi^{3}|\psi|^{2} \mathrm{~d} x \mathrm{~d} t \\
& -4 s \lambda^{2} \iint_{Q} \xi\left|\nabla^{t} \psi \nabla \eta^{0}\right|^{2} \mathrm{~d} x \mathrm{~d} t-12 s^{2} \lambda^{2} \iint_{Q} \xi^{2}\left|\nabla^{t} \psi \nabla \eta^{0}\right|^{2} \mathrm{~d} x \mathrm{~d} t \\
& +2\left(\left(M_{1} \psi\right)_{2},\left(M_{2} \psi\right)_{3}\right)_{L^{2}}(Q)^{N}+2\left(\left(M_{1} \psi\right)_{4},\left(M_{2} \psi\right)_{3}\right)_{L^{2}(Q)^{N}} \\
& -C\left(s \lambda \iint_{Q} \xi|\nabla \psi|^{2} \mathrm{~d} x \mathrm{~d} t+s \lambda^{3}\left(s+s^{2}+\lambda+s \lambda\right) \iint_{Q} \xi^{3}|\psi|^{2} \mathrm{~d} x \mathrm{~d} t\right. \\
& \left.+s T^{7} \mathrm{e}^{2 \lambda\left\|\eta^{0}\right\| \infty}\left(T^{7}+\lambda T^{8}+\lambda^{2} T^{8}+s \lambda+s \lambda^{2}\right) \iint_{Q} \xi^{3}|\psi|^{2} \mathrm{~d} x \mathrm{~d} t\right) . \\
& +
\end{aligned}
$$

From this inequality, we see that we have two leading positive terms in the right hand side, namely:

$$
2 s^{3} \lambda^{4} \iint_{Q}\left|\nabla \eta^{0}\right|^{4} \xi^{3}|\psi|^{2} \mathrm{~d} x \mathrm{~d} t \quad \text { and } \quad 2 s \lambda^{2} \iint_{Q}\left|\nabla \eta^{0}\right|^{2} \xi|\nabla \psi|^{2} \mathrm{~d} x \mathrm{~d} t .
$$


By virtue of the properties satisfied by $\eta^{0}$, we find

$$
s^{3} \lambda^{4} \iint_{Q}\left|\nabla \eta^{0}\right|^{4} \xi^{3}|\psi|^{2} \mathrm{~d} x \mathrm{~d} t \geq C s^{3} \lambda^{4}\left(\iint_{Q} \xi^{3}|\psi|^{2} \mathrm{~d} x \mathrm{~d} t-\iint_{\omega^{\prime} \times(0, T)} \xi^{3}|\psi|^{2} \mathrm{~d} x \mathrm{~d} t\right)
$$

and

$$
s \lambda^{2} \iint_{Q}\left|\nabla \eta^{0}\right|^{2} \xi|\nabla \psi|^{2} \mathrm{~d} x \mathrm{~d} t \geq C s \lambda^{2}\left(\iint_{Q} \xi|\nabla \psi|^{2} \mathrm{~d} x \mathrm{~d} t-\iint_{\omega^{\prime} \times(0, T)} \xi|\nabla \psi|^{2} \mathrm{~d} x \mathrm{~d} t\right) .
$$

In both cases, the global terms will stay in the left hand side, while the local ones will pass to the right.

Let us now make a proper choice of $s$ and $\lambda$, so that the last integrals appearing in (57) are absorbed by the two global terms (with $s \lambda^{2}$ and $s^{3} \lambda^{4}$ ) we have kept in the left hand side. More precisely, let us take $\lambda \geq C\left(\Omega, \omega^{\prime}\right)$ and $s \geq C \mathrm{e}^{2 \lambda\left\|\eta^{0}\right\|_{\infty}}\left(T^{7}+T^{8}\right)$. This way, we have

$$
\begin{aligned}
& C\left(s \lambda \iint_{Q} \xi|\nabla \psi|^{2} \mathrm{~d} x \mathrm{~d} t+s \lambda^{3}\left(s+s^{2}+\lambda+s \lambda\right) \iint_{Q} \xi^{3}|\psi|^{2} \mathrm{~d} x \mathrm{~d} t\right. \\
& \left.+s T^{7} \mathrm{e}^{(3 \lambda / 4)\left\|\eta^{0}\right\|_{\infty}}\left(T^{7}+\lambda T^{8}+\lambda^{2} T^{8}+s \lambda+s \lambda^{2}\right) \iint_{Q} \xi^{3}|\psi|^{2} \mathrm{~d} x \mathrm{~d} t\right) \\
& \leq \delta\left(s^{3} \lambda^{4} \iint_{Q} \xi^{3}|\psi|^{2} \mathrm{~d} x \mathrm{~d} t+s \lambda^{2} \iint_{Q} \xi|\nabla \psi|^{2} \mathrm{~d} x \mathrm{~d} t\right)
\end{aligned}
$$

for a constant $\delta(\Omega, \omega)>0$ small enough.

Consequently, we obtain from (57)

$$
\begin{aligned}
& 2\left(M_{1} \psi, M_{2} \psi\right)_{L^{2}(Q)^{N}} \geq 2 \iint_{\Sigma}\left(\left(\nabla \psi+\nabla^{t} \psi\right) \cdot n\right)_{t g} \cdot \psi_{t} \mathrm{~d} \sigma \mathrm{d} t \\
& -4 s \lambda \iint_{\Sigma} \xi \frac{\partial \eta^{0}}{\partial n}\left(\left(\nabla \psi+\nabla^{t} \psi\right) \cdot n\right) \cdot(\nabla \psi \cdot n) \mathrm{d} \sigma \mathrm{d} t \\
& +2 s \lambda \iint_{\Sigma} \xi \frac{\partial \eta^{0}}{\partial n}|\nabla \psi|^{2} \mathrm{~d} \sigma \mathrm{d} t+4 s \lambda \iint_{\Sigma} \xi \frac{\partial \eta^{0}}{\partial n} \nabla \psi: \nabla^{t} \psi \mathrm{d} \sigma \mathrm{d} t \\
& \quad-4 s \lambda \iint_{\Sigma} \xi \frac{\partial \eta^{0}}{\partial n}(\nabla \psi \cdot n) \cdot\left(\nabla^{t} \psi \cdot n\right) \mathrm{d} \sigma \mathrm{d} t \\
& -4 s \lambda \iint_{\Sigma} \xi \frac{\partial \eta^{0}}{\partial n}\left(\left(\nabla \psi+\nabla^{t} \psi\right) \cdot n\right) \cdot\left(\nabla^{t} \psi \cdot n\right) \mathrm{d} \sigma \mathrm{d} t \\
& \quad-4 s \lambda^{2} \iint_{\Sigma} \xi\left|\frac{\partial \eta^{0}}{\partial n}\right|^{2}\left(\left(\nabla \psi+\nabla^{t} \psi\right) \cdot n\right)_{t g} \cdot \psi \mathrm{d} \sigma \mathrm{d} t \\
& -2 s^{3} \lambda^{3} \iint_{\Sigma} \xi^{3}\left(\frac{\partial \eta^{0}}{\partial n}\right)^{3}|\psi|^{2} \mathrm{~d} x \mathrm{~d} t-2 s^{2} \lambda \iint_{\Sigma} \frac{\partial \eta^{0}}{\partial n} \xi \alpha_{t}|\psi|^{2} \mathrm{~d} x \mathrm{~d} t \\
& +8 s \lambda \iint_{\Sigma} \xi \frac{\partial \eta^{0}}{\partial n}\left|\nabla^{t} \psi \cdot n\right|^{2} \mathrm{~d} \sigma \mathrm{d} t+4 s^{3} \lambda^{4} \iint_{Q}\left|\nabla \eta^{0}\right|^{2} \xi^{3}\left|\psi \cdot \nabla \eta^{0}\right|^{2} \mathrm{~d} x \mathrm{~d} t \\
& +s \lambda^{2} \iint_{Q} \xi|\nabla \psi|^{2} \mathrm{~d} x \mathrm{~d} t+s^{3} \lambda^{4} \iint_{Q} \xi^{3}|\psi|^{2} \mathrm{~d} x \mathrm{~d} t \\
& \quad-4 s \lambda^{2} \iint_{Q} \xi\left|\nabla^{t} \psi \nabla \eta^{0}\right|^{2} \mathrm{~d} x \mathrm{~d} t-12 s^{2} \lambda^{2} \iint_{Q} \xi^{2}\left|\nabla^{t} \psi \nabla \eta^{0}\right|^{2} \mathrm{~d} x \mathrm{~d} t \\
& +2\left(\left(M_{1} \psi\right)_{2},\left(M_{2} \psi\right)_{3}\right)_{L^{2}(Q)^{N}}+2\left(\left(M_{1} \psi\right)_{4},\left(M_{2} \psi\right)_{3}\right)_{L^{2}(Q)^{N}}
\end{aligned}
$$

for any $\lambda \geq C(\Omega, \omega)$ and any $s \geq C(\Omega, \omega) \mathrm{e}^{2 \lambda\left\|\eta^{0}\right\|_{\infty}}\left(T^{7}+T^{8}\right)$. 
On the other hand, we also have several negative terms (in $Q$ ) with high powers of the parameters $s$ and $\lambda$. Those will be eliminated with the use of the positive terms appearing in the left hand side of (50), i.e.,

$$
\left\|M_{1} \psi\right\|_{L^{2}(Q)^{N}}^{2} \quad \text { and } \quad\left\|M_{2} \psi\right\|_{L^{2}(Q)^{N}}^{2}
$$

Let us also observe that

$$
\left\|G_{s, \lambda}\right\|_{L^{2}(Q)^{N}}^{2} \leq C\left(\iint_{Q} \mathrm{e}^{-2 s \alpha}|G|^{2} \mathrm{~d} x \mathrm{~d} t+s^{2} \lambda^{4} \iint_{Q} \xi^{2}|\psi|^{2} \mathrm{~d} x \mathrm{~d} t\right),
$$

for $\lambda \geq C(\Omega, \omega)$.

Therefore, from (50) and (58), we readily get:

$$
\begin{aligned}
& \left\|M_{1} \psi\right\|_{L^{2}(Q)^{N}}^{2}+\left\|M_{2} \psi\right\|_{L^{2}(Q)^{N}}^{2}+s^{3} \lambda^{4} \iint_{Q} \xi^{3}|\psi|^{2} \mathrm{~d} x \mathrm{~d} t \\
& +s \lambda^{2} \iint_{Q} \xi|\nabla \psi|^{2} \mathrm{~d} x \mathrm{~d} t+4 s^{3} \lambda^{4} \iint_{Q}\left|\nabla \eta^{0}\right|^{2} \xi^{3}\left|\psi \cdot \nabla \eta^{0}\right|^{2} \mathrm{~d} x \mathrm{~d} t \\
& -4 s \lambda^{2} \iint_{Q} \xi\left|\nabla^{t} \psi \nabla \eta^{0}\right|^{2} \mathrm{~d} x \mathrm{~d} t-12 s^{2} \lambda^{2} \iint_{Q} \xi^{2}\left|\nabla^{t} \psi \nabla \eta^{0}\right|^{2} \mathrm{~d} x \mathrm{~d} t \\
& +2\left(\left(M_{1} \psi\right)_{2},\left(M_{2} \psi\right)_{3}\right)_{L^{2}(Q)^{N}}+2\left(\left(M_{1} \psi\right)_{4},\left(M_{2} \psi\right)_{3}\right)_{L^{2}(Q)^{N}} \\
& +2 \iint_{\Sigma}\left(\left(\nabla \psi+\nabla^{t} \psi\right) \cdot n\right)_{t g} \cdot \psi_{t} \mathrm{~d} \sigma \mathrm{d} t \\
& -4 s \lambda \iint_{\Sigma} \xi \frac{\partial \eta^{0}}{\partial n}\left(\left(\nabla \psi+\nabla^{t} \psi\right) \cdot n\right) \cdot(\nabla \psi \cdot n) \mathrm{d} \sigma \mathrm{d} t \\
& +2 s \lambda \iint_{\Sigma} \xi \frac{\partial \eta^{0}}{\partial n}|\nabla \psi|^{2} \mathrm{~d} \sigma \mathrm{d} t+4 s \lambda \iint_{\Sigma} \xi \frac{\partial \eta^{0}}{\partial n} \nabla \psi: \nabla^{t} \psi \mathrm{d} \sigma \mathrm{d} t \\
& -4 s \lambda \iint_{\Sigma} \xi \frac{\partial \eta^{0}}{\partial n}(\nabla \psi \cdot n) \cdot\left(\nabla^{t} \psi \cdot n\right) \mathrm{d} \sigma \mathrm{d} t \\
& -4 s \lambda \iint_{\Sigma} \xi \frac{\partial \eta^{0}}{\partial n}\left(\left(\nabla \psi+\nabla^{t} \psi\right) \cdot n\right) \cdot\left(\nabla^{t} \psi \cdot n\right) \mathrm{d} \sigma \mathrm{d} t \\
& -4 s \lambda^{2} \iint_{\Sigma} \xi\left|\frac{\partial \eta^{0}}{\partial n}\right|^{2}\left(\left(\nabla \psi+\nabla^{t} \psi\right) \cdot n\right)_{t g} \cdot \psi \mathrm{d} \sigma \mathrm{d} t \\
& -2 s^{3} \lambda^{3} \iint_{\Sigma} \xi^{3}\left(\frac{\partial \eta^{0}}{\partial n}\right)^{3}|\psi|^{2} \mathrm{~d} \sigma \mathrm{d} t-2 s^{2} \lambda \iint_{\Sigma} \frac{\partial \eta^{0}}{\partial n} \xi \alpha_{t}|\psi|^{2} \mathrm{~d} \sigma \mathrm{d} t \\
& +8 s \lambda \iint_{\Sigma} \xi \frac{\partial \eta^{0}}{\partial n}\left|\nabla^{t} \psi \cdot n\right|^{2} \mathrm{~d} \sigma \mathrm{d} t \\
& \leq C\left(\iint_{Q} \mathrm{e}^{-2 s \alpha}|G|^{2} \mathrm{~d} x \mathrm{~d} t+s^{3} \lambda^{4} \iint_{\omega^{\prime} \times(0, T)} \xi^{3}|\psi|^{2} \mathrm{~d} x \mathrm{~d} t\right. \\
& \left.+s \lambda^{2} \iint_{\omega^{\prime} \times(0, T)} \xi|\nabla \psi|^{2} \mathrm{~d} x \mathrm{~d} t\right),
\end{aligned}
$$

for any $\lambda \geq C(\Omega, \omega)$ and any $s \geq C(\Omega, \omega) \mathrm{e}^{2 \lambda\left\|\eta^{0}\right\|_{\infty}}\left(T^{7}+T^{8}\right)$. 
At this point, let us develop the term $M_{1} \psi$ in the following way:

$$
M_{1} \psi=\bar{M}_{1} \psi-2 s \lambda \xi \nabla^{t} \psi \cdot \nabla \eta^{0}
$$

with

$$
\bar{M}_{1} \psi=-\Delta \psi-s^{2} \lambda^{2}\left|\nabla \eta^{0}\right|^{2} \xi^{2} \psi-s \alpha_{t} \psi-s \lambda \xi\left(\nabla \nabla \eta^{0} \cdot \psi\right)-s \lambda^{2} \nabla \eta^{0} \xi\left(\psi \cdot \nabla \eta^{0}\right)
$$

that is to say,

$$
\bar{M}_{1} \psi=-\Delta \psi+\left(M_{1} \psi\right)_{2}+\left(M_{1} \psi\right)_{4}-s \lambda \xi\left(\nabla \nabla \eta^{0} \cdot \psi\right)-s \lambda^{2} \nabla \eta^{0} \xi\left(\psi \cdot \nabla \eta^{0}\right) .
$$

Hence,

$$
\left\|M_{1} \psi\right\|_{L^{2}(Q)^{N}}^{2}=\left\|\bar{M}_{1} \psi\right\|_{L^{2}(Q)^{N}}^{2}+4 s^{2} \lambda^{2} \iint_{Q} \xi^{2}\left|\nabla^{t} \psi \cdot \nabla \eta^{0}\right|^{2} \mathrm{~d} x \mathrm{~d} t+2\left(\bar{M}_{1} \psi,-2 s \lambda \xi\left(\nabla^{t} \psi \cdot \nabla \eta^{0}\right)\right)_{L^{2}(Q)^{N}}
$$

Let us deal with the double product term. To this end, we first observe that it actually coincides with

$$
\begin{aligned}
2\left(\bar{M}_{1} \psi,-\left(M_{2} \psi\right)_{3}\right)_{L^{2}(Q)^{N}=} & -2\left(\left(M_{1} \psi\right)_{2},\left(M_{2} \psi\right)_{3}\right)_{L^{2}(Q)^{N}}-2\left(\left(M_{1} \psi\right)_{4},\left(M_{2} \psi\right)_{3}\right)_{L^{2}(Q)^{N}} \\
& +4 s \lambda \iint_{Q} \xi\left(\nabla^{t} \psi \cdot \nabla \eta^{0}\right) \cdot \Delta \psi \mathrm{d} x \mathrm{~d} t \\
& +4 s^{2} \lambda^{2} \iint_{Q} \xi^{2}\left(\nabla \nabla \eta^{0} \cdot \psi\right) \cdot\left(\nabla^{t} \psi \cdot \nabla \eta^{0}\right) \mathrm{d} x \mathrm{~d} t \\
& +4 s^{2} \lambda^{3} \iint_{Q} \xi^{2}\left(\psi \cdot \nabla \eta^{0}\right) \nabla \eta^{0} \cdot\left(\nabla^{t} \psi \cdot \nabla \eta^{0}\right) \mathrm{d} x \mathrm{~d} t \\
= & -2\left(\left(M_{1} \psi\right)_{2},\left(M_{2} \psi\right)_{3}\right)-2\left(\left(M_{1} \psi\right)_{4},\left(M_{2} \psi\right)_{3}\right)_{L^{2}(Q)^{N}}+\mathcal{T}-H_{41}+2 E_{244}
\end{aligned}
$$

The next step will be to estimate the third term in the previous expression:

$$
\begin{array}{r}
\mathcal{T}=-4 s \lambda \iint_{Q} \xi\left(\nabla \nabla \eta^{0} \cdot \psi\right) \cdot \Delta \psi \mathrm{d} x \mathrm{~d} t-4 s \lambda^{2} \iint_{Q} \xi\left(\psi \cdot \nabla \eta^{0}\right)\left(\Delta \psi \cdot \nabla \eta^{0}\right) \mathrm{d} x \mathrm{~d} t \\
\quad-4 s^{2} \lambda^{2} \iint_{Q} \xi\left(\psi \cdot \nabla \eta^{0}\right) \Delta\left(\xi \psi \cdot \nabla \eta^{0}\right) \mathrm{d} x \mathrm{~d} t=\mathcal{T}_{1}+\mathcal{T}_{2}+\mathcal{T}_{3}
\end{array}
$$

For $\mathcal{T}_{1}$, we have

$$
\mathcal{T}_{1} \leq C s \lambda \iint_{Q} \xi|\psi||\Delta \psi| \mathrm{d} x \mathrm{~d} t
$$

while we must still arrange $\mathcal{T}_{2}$ and $\mathcal{T}_{3}$, making some integration by parts. Indeed, we have

$$
\begin{aligned}
\mathcal{T}_{2}= & 4 s \lambda^{2} \iint_{Q} \xi\left(\nabla\left(\nabla \eta^{0} \nabla \eta^{0}\right) \cdot \nabla \psi\right) \cdot \psi \mathrm{d} x \mathrm{~d} t \\
& +4 s \lambda^{3} \iint_{Q} \xi\left(\psi \cdot \nabla \eta^{0}\right)\left(\nabla \psi \cdot \nabla \eta^{0}\right) \cdot \nabla \eta^{0} \mathrm{~d} x \mathrm{~d} t \\
& +4 s \lambda^{2} \iint_{Q} \xi\left|\nabla^{t} \psi \cdot \nabla \eta^{0}\right|^{2} \mathrm{~d} x \mathrm{~d} t=\mathcal{T}_{21}+\mathcal{T}_{22}+\mathcal{T}_{23}
\end{aligned}
$$


$\mathcal{T}_{23}$ will compensate the sixth term in the left hand side of (59) and $\mathcal{T}_{21}, \mathcal{T}_{22}$ verify

$$
\mathcal{T}_{21}+\mathcal{T}_{22} \leq C\left(s^{2} \lambda^{4} \iint_{Q} \xi^{2}|\psi|^{2} \mathrm{~d} x \mathrm{~d} t+\left(1+\lambda^{2}\right) \iint_{Q} \xi|\nabla \psi|^{2} \mathrm{~d} x \mathrm{~d} t\right)
$$

so they will be eliminated by taking $\lambda \geq 1$ and $s \geq C T^{8}$. Besides,

$$
\begin{aligned}
\mathcal{T}_{3}= & 4 s^{2} \lambda^{2} \iint_{Q} \xi\left(\nabla \nabla \eta^{0} \cdot \psi\right) \cdot \nabla\left(\xi \psi \cdot \nabla \eta^{0}\right) \mathrm{d} x \mathrm{~d} t \\
& +2 s^{2} \lambda^{3} \iint_{Q} \nabla \eta^{0} \cdot \nabla\left|\xi \psi \cdot \nabla \eta^{0}\right|^{2} \mathrm{~d} x \mathrm{~d} t \\
& +4 s^{2} \lambda^{2} \iint_{Q} \xi\left(\nabla^{t} \psi \cdot \nabla \eta^{0}\right) \cdot \nabla\left(\xi \psi \cdot \nabla \eta^{0}\right) \mathrm{d} x \mathrm{~d} t=\mathcal{T}_{31}+\mathcal{T}_{32}+\mathcal{T}_{33} .
\end{aligned}
$$

For $\mathcal{T}_{31}$ and $\mathcal{T}_{32}$ (after integration by parts), we have

$$
\mathcal{T}_{31}+\mathcal{T}_{32} \leq C\left(s^{2} \lambda^{2}(1+s+\lambda) \iint_{Q} \xi^{2}|\psi|^{2} \mathrm{~d} x \mathrm{~d} t+s \lambda \iint_{Q} \xi|\nabla \psi|^{2} \mathrm{~d} x \mathrm{~d} t\right)
$$

which are 'good' terms, provided we make a good choice of $s$ and $\lambda$. For $\mathcal{T}_{33}$, we find

$$
\begin{aligned}
\mathcal{T}_{33}= & 4 s^{2} \lambda^{2} \iint_{Q} \xi^{2}\left(\nabla^{t} \psi \cdot \nabla \eta^{0}\right) \cdot\left(\nabla \nabla \eta^{0} \cdot \psi\right) \mathrm{d} x \mathrm{~d} t \\
& +4 s^{2} \lambda^{3} \iint_{Q} \xi^{2}\left(\nabla^{t} \psi \cdot \nabla \eta^{0}\right) \cdot \nabla \eta^{0}\left(\psi \cdot \nabla \eta^{0}\right) \mathrm{d} x \mathrm{~d} t \\
& +4 s^{2} \lambda^{2} \iint_{Q} \xi^{2}\left|\nabla^{t} \psi \cdot \nabla \eta^{0}\right|^{2} \mathrm{~d} x \mathrm{~d} t=-H_{41}+2 E_{244}+\mathcal{T}_{331} .
\end{aligned}
$$

Consequently,

$$
\mathcal{T}_{33} \geq-C\left(s^{2} \lambda^{3}(1+\lambda) \iint_{Q} \xi^{3}|\psi|^{2} \mathrm{~d} x \mathrm{~d} t+s \lambda \iint_{Q} \xi|\nabla \psi|^{2} \mathrm{~d} x \mathrm{~d} t\right)+\mathcal{T}_{331}
$$


Combining all this and (59), we deduce the following inequality:

$$
\begin{aligned}
& \left\|\bar{M}_{1} \psi\right\|_{L^{2}(Q)^{N}}^{2}+\left\|M_{2} \psi\right\|_{L^{2}(Q)^{N}}^{2}+s^{3} \lambda^{4} \iint_{Q} \xi^{3}|\psi|^{2} \mathrm{~d} x \mathrm{~d} t \\
& +s \lambda^{2} \iint_{Q} \xi|\nabla \psi|^{2} \mathrm{~d} x \mathrm{~d} t+4 s^{3} \lambda^{4} \iint_{Q}\left|\nabla \eta^{0}\right|^{2} \xi^{3}\left|\psi \cdot \nabla \eta^{0}\right|^{2} \mathrm{~d} x \mathrm{~d} t \\
& -4 s^{2} \lambda^{2} \iint_{Q} \xi^{2}\left|\nabla^{t} \psi \nabla \eta^{0}\right|^{2} \mathrm{~d} x \mathrm{~d} t+2 \iint_{\Sigma}\left(\left(\nabla \psi+\nabla^{t} \psi\right) \cdot n\right)_{t g} \cdot \psi_{t} \mathrm{~d} \sigma \mathrm{d} t \\
& -4 s \lambda \iint_{\Sigma} \xi \frac{\partial \eta^{0}}{\partial n}\left(\left(\nabla \psi+\nabla^{t} \psi\right) \cdot n\right) \cdot(\nabla \psi \cdot n) \mathrm{d} \sigma \mathrm{d} t \\
& +2 s \lambda \iint_{\Sigma} \xi \frac{\partial \eta^{0}}{\partial n}|\nabla \psi|^{2} \mathrm{~d} \sigma \mathrm{d} t+4 s \lambda \iint_{\Sigma} \xi \frac{\partial \eta^{0}}{\partial n} \nabla \psi: \nabla^{t} \psi \mathrm{d} \sigma \mathrm{d} t \\
& -4 s \lambda \iint_{\Sigma} \xi \frac{\partial \eta^{0}}{\partial n}(\nabla \psi \cdot n) \cdot\left(\nabla^{t} \psi \cdot n\right) \mathrm{d} \sigma \mathrm{d} t \\
& -4 s \lambda \iint_{\Sigma} \xi \frac{\partial \eta^{0}}{\partial n}\left(\left(\nabla \psi+\nabla^{t} \psi\right) \cdot n\right) \cdot\left(\nabla^{t} \psi \cdot n\right) \mathrm{d} \sigma \mathrm{d} t \\
& -4 s \lambda^{2} \iint_{\Sigma} \xi\left|\frac{\partial \eta^{0}}{\partial n}\right|^{2}\left(\left(\nabla \psi+\nabla^{t} \psi\right) \cdot n\right)_{t g} \cdot \psi \mathrm{d} \sigma \mathrm{d} t \\
& \left.\quad+s^{3} \lambda^{4} \iint_{\omega^{\prime} \times(0, T)} \xi^{3}|\psi|^{2} \mathrm{~d} x \mathrm{~d} t+s \lambda^{2} \iint_{\omega^{\prime} \times(0, T)} \xi|\nabla \psi|^{2} \mathrm{~d} x \mathrm{~d} t\right), \\
& \quad \iint_{\Sigma} \xi^{3}\left(\frac{\partial \eta^{0}}{\partial n}\right)^{3}|\psi|^{2} \mathrm{~d} \sigma \mathrm{d} t-2 s^{2} \lambda \iint_{\Sigma} \frac{\partial \eta^{0}}{\partial n} \xi \alpha_{t}|\psi|^{2} \mathrm{~d} \sigma \mathrm{d} t \\
& +8 s \lambda \iint_{\Sigma} \xi \frac{\partial \eta^{0}}{\partial n}\left|\nabla^{t} \psi \cdot n\right|^{2} \mathrm{~d} \sigma \mathrm{d} t \\
& \quad \leq \int_{Q} \mathrm{e}^{-2 s \alpha}|G|^{2} \mathrm{~d} x \mathrm{~d} t+s \lambda \iint_{Q} \xi|\psi \| \Delta \psi| \mathrm{d} x \mathrm{~d} t
\end{aligned}
$$

for any $\lambda \geq C(\Omega, \omega)$ and any $s \geq C(\Omega, \omega) \mathrm{e}^{2 \lambda\left\|\eta^{0}\right\|_{\infty}}\left(T^{7}+T^{8}\right)$.

Let us finally expand the term $M_{2} \psi$ as follows:

$$
M_{2} \psi=\bar{M}_{2} \psi+2 s \lambda \xi \nabla^{t} \psi \cdot \nabla \eta^{0}
$$

with

$$
\bar{M}_{2} \psi=\left(M_{2} \psi\right)_{1}+\left(M_{2} \psi\right)_{2}+\left(M_{2} \psi\right)_{4} .
$$

This way, $\left\|M_{2} \psi\right\|_{L^{2}(Q)^{N}}^{2}$ can be written as follows:

$$
\begin{aligned}
\left\|M_{2} \psi\right\|_{L^{2}(Q)^{N}}^{2}= & 4 s^{2} \lambda^{2} \iint_{Q} \xi^{2}\left|\nabla^{t} \psi \cdot \nabla \eta^{0}\right|^{2} \mathrm{~d} x \mathrm{~d} t+\left\|\bar{M}_{2} \psi\right\|_{L^{2}(Q)^{N}}^{2} \\
& -4\left[\left(\left(M_{1} \psi\right)_{3},\left(M_{2} \psi\right)_{1}\right)_{L^{2}(Q)^{N}}+\left(\left(M_{1} \psi\right)_{3},\left(M_{2} \psi\right)_{2}\right)_{L^{2}(Q)^{N}}\right. \\
& \left.+\left(\left(M_{1} \psi\right)_{3},\left(M_{2} \psi\right)_{4}\right)_{L^{2}(Q)^{N}}\right] .
\end{aligned}
$$

Coming back to the computations we have already made for these products, we have:

$$
\begin{aligned}
-4\left[\left(\left(M_{1} \psi\right)_{3},\left(M_{2} \psi\right)_{1}\right.\right. & \left.\left.\left.+\left(M_{2} \psi\right)_{2}+\left(M_{2} \psi\right)_{4}\right)\right)_{L^{2}(Q)^{N}}\right] \\
& =-4\left(M_{1}+M_{2}+N_{1}+2 E_{244}+N_{21}+N_{22}+P_{1}+P_{2}\right) .
\end{aligned}
$$


Making now the same computations we did when we deduce (54), we find

$$
\begin{aligned}
\left\|M_{2} \psi\right\|_{L^{2}(Q)^{N}}^{2} \geq & 4 s^{2} \lambda^{2} \iint_{Q} \xi^{2}\left|\nabla^{t} \psi \cdot \nabla \eta^{0}\right|^{2} \mathrm{~d} x \mathrm{~d} t+\left\|\bar{M}_{2} \psi\right\|_{L^{2}(Q)^{N}}^{2} \\
& -4 s^{3} \lambda^{4} \iint_{Q}\left|\nabla \eta^{0}\right|^{2} \xi^{3}\left|\psi \cdot \nabla \eta^{0}\right|^{2} \mathrm{~d} x \mathrm{~d} t \\
& -C\left(s \lambda T(1+\lambda) \iint_{Q} \xi^{5 / 4}|\psi|^{2} \mathrm{~d} x \mathrm{~d} t+s \lambda \iint_{Q} \xi|\nabla \psi|^{2} \mathrm{~d} x \mathrm{~d} t\right. \\
& \left.+s^{3} \lambda^{3} \iint_{Q} \xi^{3}|\psi|^{2} \mathrm{~d} x \mathrm{~d} t+s^{2} \lambda^{3}(1+\lambda) \iint_{Q} \xi^{2}|\psi|^{2} \mathrm{~d} x \mathrm{~d} t\right) .
\end{aligned}
$$

This, together with (60), provides the desired Carleman estimate for $\psi$ we wanted in the Step 1, say:

$$
\begin{aligned}
& \left\|\bar{M}_{1} \psi\right\|_{L^{2}(Q)^{N}}^{2}+\left\|\bar{M}_{2} \psi\right\|_{L^{2}(Q)^{N}}^{2}+s^{3} \lambda^{4} \iint_{Q} \xi^{3}|\psi|^{2} \mathrm{~d} x \mathrm{~d} t \\
& +s \lambda^{2} \iint_{Q} \xi|\nabla \psi|^{2} \mathrm{~d} x \mathrm{~d} t+2 \iint_{\Sigma}\left(\left(\nabla \psi+\nabla^{t} \psi\right) \cdot n\right)_{t g} \cdot \psi_{t} \mathrm{~d} \sigma \mathrm{d} t \\
& -4 s \lambda \iint_{\Sigma} \xi \frac{\partial \eta^{0}}{\partial n}\left(\left(\nabla \psi+\nabla^{t} \psi\right) \cdot n\right) \cdot(\nabla \psi \cdot n) \mathrm{d} \sigma \mathrm{d} t \\
& +2 s \lambda \iint_{\Sigma} \xi \frac{\partial \eta^{0}}{\partial n}|\nabla \psi|^{2} \mathrm{~d} \sigma \mathrm{d} t+4 s \lambda \iint_{\Sigma} \xi \frac{\partial \eta^{0}}{\partial n} \nabla \psi: \nabla^{t} \psi \mathrm{d} \sigma \mathrm{d} t \\
& -4 s \lambda \iint_{\Sigma} \xi \frac{\partial \eta^{0}}{\partial n}(\nabla \psi \cdot n) \cdot\left(\nabla^{t} \psi \cdot n\right) \mathrm{d} \sigma \mathrm{d} t \\
& -4 s \lambda \iint_{\Sigma} \xi \frac{\partial \eta^{0}}{\partial n}\left(\left(\nabla \psi+\nabla^{t} \psi\right) \cdot n\right) \cdot\left(\nabla^{t} \psi \cdot n\right) \mathrm{d} \sigma \mathrm{d} t \\
& -\left.4 s \lambda^{2} \iint_{\Sigma} \xi \frac{\partial \eta^{0}}{\partial n}\right|^{2}\left(\left(\nabla \psi+\nabla^{t} \psi\right) \cdot n\right)_{t g} \cdot \psi \mathrm{d} \sigma \mathrm{d} t \\
& -2 s^{3} \lambda^{3} \iint_{\Sigma} \xi^{3}\left(\frac{\partial \eta^{0}}{\partial n}\right)^{3}|\psi|^{2} \mathrm{~d} \sigma \mathrm{d} t-2 s^{2} \lambda \iint_{\Sigma} \frac{\partial \eta^{0}}{\partial n} \xi \alpha_{t}|\psi|^{2} \mathrm{~d} \sigma \mathrm{d} t \\
& \quad+8 s \lambda \iint_{\Sigma} \xi \frac{\partial \eta^{0}}{\partial n}\left|\nabla^{t} \psi \cdot n\right|^{2} \mathrm{~d} \sigma \mathrm{d} t \\
& \quad \leq C\left(\iint_{Q} \mathrm{e}^{-2 s \alpha}|G|^{2} \mathrm{~d} x \mathrm{~d} t+s \lambda \iint_{Q} \xi|\psi \| \Delta \psi| \mathrm{d} x \mathrm{~d} t\right. \\
& \left.\quad \leq \int_{\omega^{\prime} \times(0, T)} \xi^{3}|\psi|^{2} \mathrm{~d} x \mathrm{~d} t+s \lambda^{2} \iint_{\omega^{\prime} \times(0, T)} \xi|\nabla \psi|^{2} \mathrm{~d} x \mathrm{~d} t\right)
\end{aligned}
$$

for any $\lambda \geq C(\Omega, \omega)$ and any $s \geq C(\Omega, \omega) \mathrm{e}^{2 \lambda\left\|\eta^{0}\right\|_{\infty}}\left(T^{7}+T^{8}\right)$.

Step 2. A Carleman inequality for $\tilde{\psi}$ :

The strategy we follow here will be analogous to that employed in the first step. Moreover, all the integration by parts will provide the same terms we obtained above up to the sign. Consequently, we will pass over the details and we will focus on the explicit expressions of the resulting integrals.

More precisely, the following equality holds:

$$
M_{3} \tilde{\psi}+M_{4} \tilde{\psi}=\tilde{G}_{s, \lambda},
$$


with

and

$$
\begin{gathered}
M_{3} \tilde{\psi}=-\nabla \cdot\left(\nabla \tilde{\psi}+\nabla^{t} \tilde{\psi}\right)-s^{2} \lambda^{2}\left|\nabla \eta^{0}\right|^{2} \tilde{\xi}^{2} \tilde{\psi}+s \lambda \tilde{\xi} \nabla^{t} \tilde{\psi} \cdot \nabla \eta^{0}-s \tilde{\alpha}_{t} \tilde{\psi} \\
M_{4} \tilde{\psi}=-\tilde{\psi}_{t}-2 s \lambda \tilde{\xi} \nabla \tilde{\psi} \cdot \nabla \eta^{0}-2 s \lambda \tilde{\xi} \nabla^{t} \tilde{\psi} \cdot \nabla \eta^{0}+2 s \lambda^{2}\left|\nabla \eta^{0}\right|^{2} \tilde{\xi} \tilde{\psi}
\end{gathered}
$$

$$
\tilde{G}_{s, \lambda}=\mathrm{e}^{-s \tilde{\alpha}} G+s \lambda \Delta \eta^{0} \tilde{\xi} \tilde{\psi}+s \lambda \tilde{\xi} \nabla \nabla \eta^{0} \cdot \tilde{\psi}+s \lambda^{2}\left|\nabla \eta^{0}\right|^{2} \tilde{\xi} \tilde{\psi}-s \lambda^{2} \nabla \eta^{0} \tilde{\xi} \nabla \eta^{0} \cdot \tilde{\psi}
$$

Similarly to (50), we find that

$$
\left\|M_{3} \tilde{\psi}\right\|_{L^{2}(Q)^{N}}^{2}+\left\|M_{4} \tilde{\psi}\right\|_{L^{2}(Q)^{N}}^{2}+2\left(M_{3} \tilde{\psi}, M_{4} \tilde{\psi}\right)_{L^{2}(Q)^{N}}=\left\|\tilde{G}_{s, \lambda}\right\|_{L^{2}(Q)^{N}}^{2}
$$

Before proceeding with the computations of the double product term, let us point out several properties which are different in this case:

First, we get

$$
\nabla \tilde{\xi}=-\lambda \nabla \eta^{0} \tilde{\xi}, \quad \nabla \cdot \tilde{\psi}=-s \lambda \tilde{\xi}\left(\tilde{\psi} \cdot \nabla \eta^{0}\right) .
$$

with

$$
\left(\left(M_{3} \tilde{\psi}\right)_{1},\left(M_{4} \tilde{\psi}\right)_{1}\right)_{L^{2}(Q)^{N}}=\tilde{A}_{1}
$$

$$
\tilde{A}_{1}=\iint_{\Sigma}\left(\left(\nabla \tilde{\psi}+\nabla^{t} \tilde{\psi}\right) \cdot n\right)_{t g} \cdot \tilde{\psi}_{t} \mathrm{~d} \sigma \mathrm{d} t
$$

Then,

with

$$
\begin{aligned}
\left(\left(M_{3} \tilde{\psi}\right)_{1},\left(M_{4} \tilde{\psi}\right)_{2}\right)_{L^{2}(Q)^{N}=} & \tilde{B}+\tilde{\mathcal{C}}+\tilde{D}_{1}+\tilde{D}_{2}+\tilde{D}_{21}+\tilde{D}_{22}+\tilde{E}_{11}+\tilde{E}_{12} \\
& +\tilde{E}_{13}+\tilde{E}_{21}+\tilde{E}_{22}+\tilde{E}_{23}+\tilde{E}_{241}+\tilde{E}_{242}+\tilde{E}_{243} \\
& -\tilde{E}_{244}+\tilde{E}_{2451}+\tilde{E}_{2452}+\tilde{E}_{2453}
\end{aligned}
$$

$$
\begin{gathered}
\tilde{B}=2 s \lambda \iint_{\Sigma} \tilde{\xi} \frac{\partial \eta^{0}}{\partial n}\left(\left(\nabla \tilde{\psi}+\nabla^{t} \tilde{\psi}\right) \cdot n\right) \cdot(\nabla \tilde{\psi} \cdot n) \mathrm{d} \sigma \mathrm{d} t \\
\tilde{\mathcal{C}}=-2 s \lambda \iint_{Q} \tilde{\xi}\left(\nabla \nabla \eta^{0} \cdot \nabla \tilde{\psi}\right):\left(\nabla \tilde{\psi}+\nabla^{t} \tilde{\psi}\right) \mathrm{d} x \mathrm{~d} t \\
\tilde{D}_{1}=2 s \lambda^{2} \iint_{Q} \tilde{\xi}\left|\nabla \tilde{\psi} \cdot \nabla \eta^{0}\right|^{2} \mathrm{~d} x \mathrm{~d} t \\
\tilde{D}_{2}=2 s \lambda^{2} \iint_{Q} \tilde{\xi}\left(\nabla^{t} \tilde{\psi} \cdot \nabla \eta^{0}\right) \cdot\left(\nabla \tilde{\psi} \cdot \nabla \eta^{0}\right) \mathrm{d} x \mathrm{~d} t \\
\tilde{D}_{21}=-2 s \lambda^{2} \iint_{Q}\left(\nabla^{t}\left(\nabla \eta^{0} \nabla \eta^{0} \tilde{\xi}\right) \cdot \nabla \tilde{\psi}\right) \cdot \tilde{\psi} \mathrm{d} x \mathrm{~d} t \\
\tilde{D}_{22}=-s^{2} \lambda^{3} \iint_{Q} \Delta \eta^{0}\left|\tilde{\xi} \nabla \eta^{0} \cdot \tilde{\psi}\right|^{2} \mathrm{~d} x \mathrm{~d} t \\
\tilde{E}_{11}=s \lambda \iint_{\Sigma} \tilde{\xi} \frac{\partial \eta^{0}}{\partial n}|\nabla \tilde{\psi}|^{2} \mathrm{~d} \sigma \mathrm{d} t, \quad \tilde{E}_{12}=s \lambda \iint_{Q} \Delta \eta^{0} \tilde{\xi}|\nabla \tilde{\psi}|^{2} \mathrm{~d} x \mathrm{~d} t \\
\tilde{E}_{13}=-s \lambda^{2} \iint_{Q}\left|\nabla \eta^{0}\right|^{2} \tilde{\xi}|\nabla \tilde{\psi}|^{2} \mathrm{~d} x \mathrm{~d} t \\
\tilde{E}_{21}=-2 s \lambda \iint_{\Sigma} \tilde{\xi} \frac{\partial \eta^{0}}{\partial n} \nabla \tilde{\psi}: \nabla^{t} \tilde{\psi} \mathrm{d} \sigma \mathrm{d} t \\
\tilde{E}_{22}=2 s \lambda \iint_{Q} \Delta \eta^{0} \tilde{\xi} \nabla \tilde{\psi}: \nabla^{t} \tilde{\psi} \mathrm{d} x \mathrm{~d} t \\
\tilde{E}_{23}=-2 s \lambda^{2} \iint_{Q}\left|\nabla \eta^{0}\right|^{2} \tilde{\xi} \nabla \tilde{\psi}: \nabla^{t} \tilde{\psi} \mathrm{d} x \mathrm{~d} t
\end{gathered}
$$




$$
\begin{gathered}
\tilde{E}_{241}=2 s \lambda \iint_{\Sigma} \tilde{\xi} \frac{\partial \eta^{0}}{\partial n}(\nabla \tilde{\psi} \cdot n) \cdot\left(\nabla^{t} \tilde{\psi} \cdot n\right) \mathrm{d} \sigma \mathrm{d} t \\
\tilde{E}_{242}=-2 s \lambda \iint_{Q} \tilde{\xi}\left(\nabla \nabla \eta^{0} \cdot \nabla^{t} \tilde{\psi}\right) \cdot \nabla \tilde{\psi} \mathrm{d} x \mathrm{~d} t \\
\tilde{E}_{243}=2 s^{2} \lambda^{2} \iint_{Q} \tilde{\xi}^{2}\left(\nabla \nabla \eta^{0} \cdot \tilde{\psi}\right) \cdot\left(\nabla \tilde{\psi} \cdot \nabla \eta^{0}\right) \mathrm{d} x \mathrm{~d} t \\
\tilde{E}_{244}=-s^{2} \lambda^{3} \iint_{Q} \tilde{\xi}^{2} \nabla \eta^{0} \cdot\left(\nabla(\tilde{\psi} \tilde{\psi}) \cdot \nabla \eta^{0} \cdot \nabla \eta^{0}\right) \mathrm{d} x \mathrm{~d} t \\
\tilde{E}_{2451}=2 s^{2} \lambda^{2} \iint_{Q} \tilde{\xi}^{2}\left(\nabla\left(\nabla \eta^{0} \nabla \eta^{0}\right) \cdot \nabla^{t} \tilde{\psi}\right) \cdot \tilde{\psi} \mathrm{d} x \mathrm{~d} t \\
\tilde{E}_{2452}=-s^{3} \lambda^{3} \iint_{Q} \Delta \eta^{0} \tilde{\xi}^{3}\left|\tilde{\psi} \cdot \nabla \eta^{0}\right|^{2} \mathrm{~d} x \mathrm{~d} t
\end{gathered}
$$

and

We also have

$$
\tilde{E}_{2453}=s^{3} \lambda^{4} \iint_{Q}\left|\nabla \eta^{0}\right|^{2} \tilde{\xi}^{3}\left|\tilde{\psi} \cdot \nabla \eta^{0}\right|^{2} \mathrm{~d} x \mathrm{~d} t .
$$

where

$$
\begin{aligned}
\left(\left(M_{3} \tilde{\psi}\right)_{1},\left(M_{4} \tilde{\psi}\right)_{3}\right)_{L^{2}(Q)^{N}} & =\tilde{D}_{22}-2 \tilde{E}_{244}+\tilde{F}_{1}+\tilde{F}_{2}+\tilde{G}_{11}+\tilde{G}_{12}+\tilde{G}_{2} \\
& +\tilde{H}_{1}+\tilde{H}_{2}+\tilde{H}_{3}+\tilde{H}_{41}+\tilde{H}_{42}
\end{aligned}
$$

$$
\begin{gathered}
\tilde{F}_{1}=-2 s \lambda \iint_{\Sigma} \tilde{\xi} \frac{\partial \eta^{0}}{\partial n}\left(\left(\nabla \tilde{\psi}+\nabla^{t} \tilde{\psi}\right) \cdot n\right) \cdot\left(\nabla^{t} \tilde{\psi} \cdot n\right) \mathrm{d} \sigma \mathrm{d} t \\
\tilde{F}_{2}=-2 s \lambda \iint_{Q} \tilde{\xi}\left(\nabla \tilde{\psi}+\nabla^{t} \tilde{\psi}\right):\left(\nabla \nabla \eta^{0} \cdot \nabla^{t} \tilde{\psi}\right) \mathrm{d} x \mathrm{~d} t \\
\tilde{G}_{11}=-2 s \lambda^{2} \iint_{Q} \tilde{\xi}\left(\nabla \nabla \eta^{0}: \nabla \tilde{\psi}\right)\left(\tilde{\psi} \cdot \nabla \eta^{0}\right) \mathrm{d} x \mathrm{~d} t \\
\tilde{G}_{12}=2 s \lambda^{3} \iint_{Q} \tilde{\xi}\left(\left(\nabla \tilde{\psi} \cdot \nabla \eta^{0}\right) \cdot \nabla \eta^{0}\right)\left(\tilde{\psi} \cdot \nabla \eta^{0}\right) \mathrm{d} x \mathrm{~d} t \\
\tilde{G}_{2}=2 s \lambda^{2} \iint_{Q} \tilde{\xi}\left|\nabla^{t} \tilde{\psi} \cdot \nabla \eta^{0}\right|^{2} \mathrm{~d} x \mathrm{~d} t \\
\tilde{H}_{1}=-4 s \lambda \iint_{\Sigma} \tilde{\xi} \frac{\partial \eta^{0}}{\partial n}\left|\nabla^{t} \tilde{\psi} \cdot n\right|^{2} \mathrm{~d} \sigma \mathrm{d} t \\
\tilde{H}_{2}=4 s \lambda \iint_{Q} \tilde{\xi}\left(\nabla \nabla \eta^{0} \cdot \nabla^{t} \tilde{\psi}\right) \cdot \nabla^{t} \tilde{\psi} \mathrm{d} x \mathrm{~d} t \\
\tilde{H}_{3}=-4 s \lambda^{2} \iint_{Q} \tilde{\xi}\left|\nabla^{t} \tilde{\psi} \cdot \nabla \eta^{0}\right|^{2} \mathrm{~d} x \mathrm{~d} t, \\
\tilde{H}_{41}=-4 s^{2} \lambda^{2} \iint_{Q} \tilde{\xi}^{2}\left(\nabla \nabla \eta^{0} \cdot \tilde{\psi}\right) \cdot\left(\nabla^{t} \tilde{\psi} \cdot \nabla \eta^{0}\right) \mathrm{d} x \mathrm{~d} t
\end{gathered}
$$

and

$$
\tilde{H}_{42}=-4 s^{2} \lambda^{2} \iint_{Q} \tilde{\xi}^{2}\left|\nabla^{t} \tilde{\psi} \cdot \nabla \eta^{0}\right|^{2} \mathrm{~d} x \mathrm{~d} t .
$$

The last double product of the first term of $M_{3} \tilde{\psi}$ is the following one:

$$
\left(\left(M_{3} \tilde{\psi}\right)_{1},\left(M_{4} \tilde{\psi}\right)_{4}\right)_{L^{2}(Q)^{N}}=\tilde{I}_{1}+\tilde{I}_{2}+\tilde{I}_{3}+\tilde{I}_{4}+\tilde{I}_{5}
$$


with

$$
\begin{gathered}
\tilde{I}_{1}=-2 s \lambda^{2} \iint_{\Sigma} \tilde{\xi}\left|\frac{\partial \eta^{0}}{\partial n}\right|^{2}\left(\left(\nabla \tilde{\psi}+\nabla^{t} \tilde{\psi}\right) \cdot n\right)_{t g} \cdot \tilde{\psi} \mathrm{d} \sigma \mathrm{d} t \\
\tilde{I}_{2}=4 s \lambda^{2} \iint_{Q} \tilde{\xi}\left(\nabla \nabla \eta^{0} \nabla \eta^{0}\right) \cdot\left(\left(\nabla \tilde{\psi}+\nabla^{t} \tilde{\psi}\right) \cdot \tilde{\psi}\right) \mathrm{d} x \mathrm{~d} t \\
\tilde{I}_{3}=-2 s \lambda^{3} \iint_{Q}\left|\nabla \eta^{0}\right|^{2} \tilde{\xi}\left(\nabla \eta^{0} \cdot\left(\nabla \tilde{\psi}+\nabla^{t} \tilde{\psi}\right)\right) \cdot \tilde{\psi} \mathrm{d} x \mathrm{~d} t \\
\tilde{I}_{4}=2 s \lambda^{2} \iint_{Q}\left|\nabla \eta^{0}\right|^{2} \tilde{\xi}|\nabla \tilde{\psi}|^{2} \mathrm{~d} x \mathrm{~d} t
\end{gathered}
$$

and

$$
\tilde{I}_{5}=2 s \lambda^{2} \iint_{Q}\left|\nabla \eta^{0}\right|^{2} \tilde{\xi} \nabla \tilde{\psi}: \nabla^{t} \tilde{\psi} \mathrm{d} x \mathrm{~d} t
$$

Equivalently to (52), we find the next inequality:

$$
\begin{aligned}
& \left(\left(M_{3} \tilde{\psi}\right)_{1}, M_{4} \tilde{\psi}\right)_{L^{2}(Q)^{N}} \geq \iint_{\Sigma}\left(\left(\nabla \tilde{\psi}+\nabla^{t} \tilde{\psi}\right) \cdot n\right)_{t g} \cdot \tilde{\psi} t \mathrm{~d} \sigma \mathrm{d} t \\
& +2 s \lambda \iint_{\Sigma} \tilde{\xi} \frac{\partial \eta^{0}}{\partial n}\left(\left(\nabla \tilde{\psi}+\nabla^{t} \tilde{\psi}\right) \cdot n\right) \cdot(\nabla \tilde{\psi} \cdot n) \mathrm{d} \sigma \mathrm{d} t \\
& \quad-s \lambda \iint_{\Sigma} \tilde{\xi} \frac{\partial \eta^{0}}{\partial n}|\nabla \tilde{\psi}|^{2} \mathrm{~d} \sigma \mathrm{d} t-2 s \lambda \iint_{\Sigma} \tilde{\xi} \frac{\partial \eta^{0}}{\partial n} \nabla \tilde{\psi}: \nabla^{t} \tilde{\psi} \mathrm{d} \sigma \mathrm{d} t \\
& \quad+2 s \lambda \iint_{\Sigma} \tilde{\xi} \frac{\partial \eta^{0}}{\partial n}(\nabla \tilde{\psi} \cdot n) \cdot\left(\nabla^{t} \tilde{\psi} \cdot n\right) \mathrm{d} \sigma \mathrm{d} t \\
& +2 s \lambda \iint_{\Sigma} \tilde{\xi} \frac{\partial \eta^{0}}{\partial n}\left(\left(\nabla \tilde{\psi}+\nabla^{t} \tilde{\psi}\right) \cdot n\right) \cdot\left(\nabla^{t} \tilde{\psi} \cdot n\right) \mathrm{d} \sigma \mathrm{d} t \\
& \quad-4 s \lambda \iint_{\Sigma} \tilde{\xi} \frac{\partial \eta^{0}}{\partial n}\left|\nabla^{t} \tilde{\psi} \cdot n\right|^{2} \mathrm{~d} \sigma \mathrm{d} t \\
& \quad-2 s \lambda^{2} \iint_{\Sigma} \tilde{\xi}\left|\frac{\partial \eta^{0}}{\partial n}\right|^{2}\left(\left(\nabla \tilde{\psi}+\nabla^{t} \tilde{\psi}\right) \cdot n\right)_{t g} \cdot \tilde{\psi} \mathrm{d} \sigma \mathrm{d} t \\
& +s \lambda^{2} \iint_{Q}\left|\nabla \eta^{0}\right|^{2} \tilde{\xi}|\nabla \tilde{\psi}|^{2} \mathrm{~d} x \mathrm{~d} t+s^{3} \lambda^{4} \iint_{Q}\left|\nabla \eta^{0}\right|^{2} \tilde{\xi}^{3}\left|\tilde{\psi} \cdot \nabla \eta^{0}\right|^{2} \mathrm{~d} x \mathrm{~d} t \\
& -2 s \lambda^{2} \iint_{Q} \tilde{\xi}\left|\nabla^{t} \tilde{\psi} \cdot \nabla \eta^{0}\right|^{2} \mathrm{~d} x \mathrm{~d} t-4 s^{2} \lambda^{2} \iint_{Q} \tilde{\xi}^{2}\left|\nabla^{t} \tilde{\psi} \cdot \nabla \eta^{0}\right|^{2} \mathrm{~d} x \mathrm{~d} t \\
& -C\left(s \lambda \iint_{Q} \tilde{\xi}|\nabla \tilde{\psi}|^{2} \mathrm{~d} x \mathrm{~d} t+s \lambda^{3}\left(s+s^{2}+\lambda\right) \iint_{Q} \tilde{\xi}^{3}|\tilde{\psi}|^{2} \mathrm{~d} x \mathrm{~d} t\right) .
\end{aligned}
$$

On the other hand, we have

$$
\left(\left(M_{3} \tilde{\psi}\right)_{2},\left(M_{4} \tilde{\psi}\right)_{1}\right)_{L^{2}(Q)^{N}}=\tilde{J}=-s^{2} \lambda^{2} \iint_{Q}\left|\nabla \eta^{0}\right|^{2} \tilde{\xi} \tilde{\xi}|\tilde{\psi}|^{2} \mathrm{~d} x \mathrm{~d} t .
$$

In addition, we obtain

$$
\left(\left(M_{3} \tilde{\psi}\right)_{2},\left(M_{4} \tilde{\psi}\right)_{2}\right)_{L^{2}(Q)^{N}}=\tilde{K}_{1}+\tilde{K}_{2}+\tilde{K}_{3}
$$


where

$$
\begin{gathered}
\tilde{K}_{1}=s^{3} \lambda^{3} \iint_{\Sigma} \tilde{\xi}^{3}\left(\frac{\partial \eta^{0}}{\partial n}\right)^{3}|\tilde{\psi}|^{2} \mathrm{~d} \sigma \mathrm{d} t, \\
\tilde{K}_{2}=-s^{3} \lambda^{3} \iint_{Q} \tilde{\xi}^{3} \nabla \cdot\left(\left|\nabla \eta^{0}\right|^{2} \nabla \eta^{0}\right)|\tilde{\psi}|^{2} \mathrm{~d} x \mathrm{~d} t
\end{gathered}
$$

and

$$
\tilde{K}_{3}=3 s^{3} \lambda^{4} \iint_{Q}\left|\nabla \eta^{0}\right|^{4} \tilde{\xi}^{3}|\tilde{\psi}|^{2} \mathrm{~d} x \mathrm{~d} t .
$$

It will not be necessary to perform the product

$$
\left(\left(M_{3} \tilde{\psi}\right)_{2},\left(M_{4} \tilde{\psi}\right)_{3}\right)_{L^{2}(Q)^{N}}
$$

neither here.

The last product for $\left(M_{3} \tilde{\psi}\right)_{2}$ yields:

$$
\left(\left(M_{3} \tilde{\psi}\right)_{2},\left(M_{4} \tilde{\psi}\right)_{4}\right)_{L^{2}(Q)^{N}}=\tilde{L}=-2 s^{3} \lambda^{4} \iint_{Q}\left|\nabla \eta^{0}\right|^{4} \tilde{\xi}^{3}|\tilde{\psi}|^{2} \mathrm{~d} x \mathrm{~d} t .
$$

Here, we must notice that

$$
\tilde{\xi}_{t} \leq C T \mathrm{e}^{(\lambda / 4)\left\|\eta^{0}\right\|_{\infty} \tilde{\xi}^{5 / 4} .}
$$

Consequently, we deduce that

$$
\begin{aligned}
& \left(\left(M_{3} \tilde{\psi}\right)_{2}, M_{4} \tilde{\psi}\right)_{L^{2}(Q)^{N}} \geq s^{3} \lambda^{3} \iint_{\Sigma} \tilde{\xi}^{3}\left(\frac{\partial \eta^{0}}{\partial n}\right)^{3}|\tilde{\psi}|^{2} \mathrm{~d} \sigma \mathrm{d} t \\
& +s^{3} \lambda^{4} \iint_{Q}\left|\nabla \eta^{0}\right|^{4} \tilde{\xi}^{3}|\tilde{\psi}|^{2} \mathrm{~d} x \mathrm{~d} t+\left(\left(M_{3}, \tilde{\psi}\right)_{2},\left(M_{4} \tilde{\psi}\right)_{3}\right)_{L^{2}(Q)^{N}} \\
& -C\left(s^{2} \lambda^{2} T \mathrm{e}^{(\lambda / 4)\left\|\eta^{0}\right\|_{\infty}} \iint_{Q} \tilde{\xi}^{9 / 4}|\tilde{\psi}|^{2} \mathrm{~d} x \mathrm{~d} t+s^{3} \lambda^{3} \iint_{Q} \tilde{\xi}^{3}|\tilde{\psi}|^{2} \mathrm{~d} x \mathrm{~d} t\right) .
\end{aligned}
$$

Let us now compute the scalar products of the third term of $M_{3} \tilde{\psi}$. Firstly, we have:

$$
\left(\left(M_{3} \tilde{\psi}\right)_{3},\left(M_{4} \tilde{\psi}\right)_{1}\right)_{L^{2}(Q)^{N}}=\tilde{M}_{1}+\tilde{M}_{2}
$$

with

$$
\tilde{M}_{1}=-\frac{1}{2} s \lambda \iint_{Q} \tilde{\xi}_{t}\left(\nabla \nabla \eta^{0} \cdot \tilde{\psi}\right) \cdot \tilde{\psi} \mathrm{d} x \mathrm{~d} t, \quad \tilde{M}_{2}=\frac{1}{2} s \lambda^{2} \iint_{Q} \tilde{\xi}_{t}\left|\tilde{\psi} \cdot \nabla \eta^{0}\right|^{2} \mathrm{~d} x \mathrm{~d} t .
$$

In addition,

where

$$
\left(\left(M_{3} \tilde{\psi}\right)_{3},\left(M_{4} \tilde{\psi}\right)_{2}\right)_{L^{2}(Q)^{N}}=2 \tilde{E}_{244}+\tilde{N}_{1}+\tilde{N}_{21}+\tilde{N}_{22},
$$

$$
\begin{gathered}
\tilde{N}_{1}=2 s^{2} \lambda^{2} \iint_{Q} \tilde{\xi}^{2}\left(\nabla^{t} \tilde{\psi} \cdot \nabla\left(\nabla \eta^{0} \nabla \eta^{0}\right)\right) \cdot \tilde{\psi} \mathrm{d} x \mathrm{~d} t, \\
\tilde{N}_{21}=s^{3} \lambda^{3} \iint_{Q} \tilde{\xi} \Delta \eta^{0}\left|\tilde{\xi} \tilde{\psi} \cdot \nabla \eta^{0}\right|^{2} \mathrm{~d} x \mathrm{~d} t
\end{gathered}
$$

and

$$
\tilde{N}_{22}=-s^{3} \lambda^{4} \iint_{Q}\left|\nabla \eta^{0}\right|^{2} \tilde{\xi}^{3}\left|\tilde{\psi} \cdot \nabla \eta^{0}\right|^{2} \mathrm{~d} x \mathrm{~d} t .
$$


Furthermore, we have

$$
\left(\left(M_{3} \tilde{\psi}\right)_{3},\left(M_{4} \tilde{\psi}\right)_{3}\right)_{L^{2}(Q)^{N}}=\tilde{O}=-2 s^{2} \lambda^{2} \iint_{Q}\left|\nabla \eta^{0}\right|^{2} \tilde{\xi}^{2}\left|\nabla^{t} \tilde{\psi} \cdot \nabla \eta^{0}\right|^{2} \mathrm{~d} x \mathrm{~d} t .
$$

The last product for $\left(M_{3} \tilde{\psi}\right)_{3}$ gives:

$$
\left(\left(M_{3} \tilde{\psi}\right)_{3},\left(M_{4} \tilde{\psi}\right)_{4}\right)_{L^{2}(Q)^{N}}=\tilde{P}_{1}+\tilde{P}_{2}
$$

where

$$
\tilde{P}_{1}=-2 s^{2} \lambda^{3} \iint_{Q}\left(\nabla\left(\left|\nabla \eta^{0}\right|^{2} \tilde{\xi}^{2} \nabla \eta^{0}\right) \cdot \tilde{\psi}\right) \cdot \tilde{\psi} \mathrm{d} x \mathrm{~d} t
$$

and

$$
\tilde{P}_{2}=2 s^{3} \lambda^{4} \iint_{Q}\left|\nabla \eta^{0}\right|^{2} \tilde{\xi}^{3}\left|\tilde{\psi} \cdot \nabla \eta^{0}\right|^{2} \mathrm{~d} x \mathrm{~d} t
$$

Combining all the products of $\left(M_{3} \tilde{\psi}\right)_{3}$, we find

$$
\begin{aligned}
& \left(\left(M_{3} \tilde{\psi}\right)_{3}, M_{4} \tilde{\psi}\right)_{L^{2}(Q)^{N}} \geq s^{3} \lambda^{4} \iint_{Q}\left|\nabla \eta^{0}\right|^{2} \tilde{\xi}^{3}\left|\tilde{\psi} \cdot \nabla \eta^{0}\right|^{2} \mathrm{~d} x \mathrm{~d} t \\
& -2 s^{2} \lambda^{2} \iint_{Q}\left|\nabla \eta^{0}\right|^{2} \tilde{\xi}^{2}\left|\nabla^{t} \tilde{\psi} \cdot \nabla \eta^{0}\right|^{2} \mathrm{~d} x \mathrm{~d} t
\end{aligned}
$$

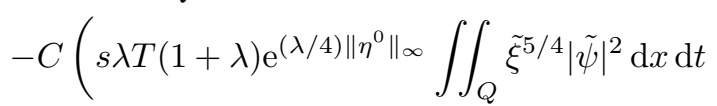

$$
\begin{aligned}
& +s \lambda \iint_{Q} \tilde{\xi}|\nabla \tilde{\psi}|^{2} \mathrm{~d} x \mathrm{~d} t+s^{3} \lambda^{3} \iint_{Q} \tilde{\xi}^{3}|\tilde{\psi}|^{2} \mathrm{~d} x \mathrm{~d} t \\
& \left.+s^{2} \lambda^{3}(1+\lambda) \iint_{Q} \tilde{\xi}^{2}|\tilde{\psi}|^{2} \mathrm{~d} x \mathrm{~d} t\right) .
\end{aligned}
$$

Let us finally consider the computations for $M_{3} \tilde{\psi}$.

First, we have:

$$
\left(\left(M_{3} \tilde{\psi}\right)_{4},\left(M_{4} \tilde{\psi}\right)_{1}\right)_{L^{2}(Q)^{N}}=\tilde{Q}=-\frac{1}{2} s \iint_{Q} \tilde{\alpha}_{t t}|\tilde{\psi}|^{2} \mathrm{~d} x \mathrm{~d} t
$$

Then,

$$
\left(\left(M_{3} \tilde{\psi}\right)_{4},\left(M_{4} \tilde{\psi}\right)_{2}\right)_{L^{2}(Q)^{N}}=\tilde{R}_{1}+\tilde{R}_{2}
$$

with

$$
\tilde{R}_{1}=s^{2} \lambda \iint_{\Sigma} \frac{\partial \eta^{0}}{\partial n} \tilde{\xi} \tilde{\alpha}_{t}|\tilde{\psi}|^{2} \mathrm{~d} \sigma \mathrm{d} t, \quad \tilde{R}_{2}=-s^{2} \lambda \iint_{Q} \nabla \cdot\left(\tilde{\xi} \tilde{\alpha}_{t} \nabla \eta^{0}\right)|\tilde{\psi}|^{2} \mathrm{~d} x \mathrm{~d} t
$$


Let us again skip the term

$$
\left(\left(M_{3} \tilde{\psi}\right)_{4},\left(M_{4} \tilde{\psi}\right)_{3}\right)_{L^{2}(Q)^{N}}
$$

Finally, we have

$$
\left(\left(M_{3} \tilde{\psi}\right)_{4},\left(M_{4} \tilde{\psi}\right)_{4}\right)_{L^{2}(Q)^{N}}=\tilde{S}=-2 s^{2} \lambda^{2} \iint_{Q}\left|\nabla \eta^{0}\right|^{2} \tilde{\xi} \tilde{\alpha}_{t}|\tilde{\psi}|^{2} \mathrm{~d} x \mathrm{~d} t
$$

This time, the expression of $\tilde{\alpha}_{t}$ is:

$$
\tilde{\alpha}_{t}=-4(T-2 t)\left(\mathrm{e}^{2 \lambda\left\|\eta^{0}\right\|_{\infty}}-\mathrm{e}^{-\lambda \eta^{0}}\right)(t(T-t))^{-5},
$$

so

$$
\left|\tilde{\alpha}_{t}\right| \leq C T \mathrm{e}^{(13 \lambda / 4)\left\|\eta^{0}\right\|_{\infty}} \tilde{\xi}^{5 / 4} .
$$

Furthermore, we have:

$$
\begin{gathered}
\left|\nabla\left(\tilde{\alpha}_{t}\right)\right| \leq C \lambda T \mathrm{e}^{(\lambda / 4)\left\|\eta^{0}\right\|_{\infty}} \tilde{\xi}^{5 / 4}, \\
\left|\tilde{\alpha}_{t t}\right| \leq C \mathrm{e}^{(13 \lambda / 4)\left\|\eta^{0}\right\|_{\infty}}\left(T^{2} \mathrm{e}^{(\lambda / 4)\left\|\eta^{0}\right\|_{\infty}} \tilde{\xi}^{3 / 2}+\tilde{\xi}^{5 / 4}\right) .
\end{gathered}
$$

Consequently, we find the following for $\left(M_{3} \tilde{\psi}\right)_{4}$ :

$$
\begin{aligned}
& \left(\left(M_{3} \tilde{\psi}\right)_{4}, M_{4} \tilde{\psi}\right)_{L^{2}(Q)^{N}} \geq s^{2} \lambda \iint_{\Sigma} \frac{\partial \eta^{0}}{\partial n} \tilde{\xi} \tilde{\alpha}_{t}|\tilde{\psi}|^{2} \mathrm{~d} \sigma \mathrm{d} t \\
& +\left(\left(M_{3} \tilde{\psi}\right)_{4},\left(M_{4} \tilde{\psi}\right)_{3}\right)_{L^{2}(Q)^{N}} \\
& -C \mathrm{e}^{(13 \lambda / 4)\left\|\eta^{0}\right\|_{\infty}}\left(s \iint_{Q}\left(T^{2} \mathrm{e}^{(\lambda / 4)\left\|\eta^{0}\right\|_{\infty}} \tilde{\xi}^{3 / 2}+\tilde{\xi}^{5 / 4}\right)|\tilde{\psi}|^{2} \mathrm{~d} x \mathrm{~d} t\right. \\
& \left.+s^{2} \lambda(1+\lambda) T \iint_{Q} \tilde{\xi}^{9 / 4}|\tilde{\psi}|^{2} \mathrm{~d} x \mathrm{~d} t\right)
\end{aligned}
$$

Therefore, taking into account (63), (64), (65), (67) and

$$
\tilde{\xi}^{-1} \leq C T^{8} \mathrm{e}^{\lambda\left\|\eta^{0}\right\|_{\infty}}
$$


we obtain

$$
\begin{aligned}
& 2\left(M_{3} \tilde{\psi}, M_{4} \tilde{\psi}\right)_{L^{2}(Q)^{N}} \geq 2 \iint_{\Sigma}\left(\left(\nabla \tilde{\psi}+\nabla^{t} \tilde{\psi}\right) \cdot n\right)_{t g} \cdot \tilde{\psi}_{t} \mathrm{~d} \sigma \mathrm{d} t \\
& +4 s \lambda \iint_{\Sigma} \tilde{\xi} \frac{\partial \eta^{0}}{\partial n}\left(\left(\nabla \tilde{\psi}+\nabla^{t} \tilde{\psi}\right) \cdot n\right) \cdot(\nabla \tilde{\psi} \cdot n) \mathrm{d} \sigma \mathrm{d} t \\
& -2 s \lambda \iint_{\Sigma} \tilde{\xi} \frac{\partial \eta^{0}}{\partial n}|\nabla \tilde{\psi}|^{2} \mathrm{~d} \sigma \mathrm{d} t-4 s \lambda \iint_{\Sigma} \tilde{\xi} \frac{\partial \eta^{0}}{\partial n} \nabla \tilde{\psi}: \nabla^{t} \tilde{\psi} \mathrm{d} \sigma \mathrm{d} t \\
& +4 s \lambda \iint_{\Sigma} \tilde{\xi} \frac{\partial \eta^{0}}{\partial n}(\nabla \tilde{\psi} \cdot n) \cdot\left(\nabla^{t} \tilde{\psi} \cdot n\right) \mathrm{d} \sigma \mathrm{d} t \\
& +4 s \lambda \iint_{\Sigma} \tilde{\xi} \frac{\partial \eta^{0}}{\partial n}\left(\left(\nabla \tilde{\psi}+\nabla^{t} \tilde{\psi}\right) \cdot n\right) \cdot\left(\nabla^{t} \tilde{\psi} \cdot n\right) \mathrm{d} \sigma \mathrm{d} t \\
& -4 s \lambda^{2} \iint_{\Sigma} \tilde{\xi}\left|\frac{\partial \eta^{0}}{\partial n}\right|^{2}\left(\left(\nabla \tilde{\psi}+\nabla^{t} \tilde{\psi}\right) \cdot n\right)_{t g} \cdot \tilde{\psi} \mathrm{d} \sigma \mathrm{d} t \\
& +2 s^{3} \lambda^{3} \iint_{\Sigma} \tilde{\xi}^{3}\left(\frac{\partial \eta^{0}}{\partial n}\right)^{3}|\tilde{\psi}|^{2} \mathrm{~d} \sigma \mathrm{d} t+2 s^{2} \lambda \iint_{\Sigma} \frac{\partial \eta^{0}}{\partial n} \tilde{\xi} \tilde{\alpha}_{t}|\tilde{\psi}|^{2} \mathrm{~d} \sigma \mathrm{d} t \\
& -8 s \lambda \iint_{\Sigma} \tilde{\xi} \frac{\partial \eta^{0}}{\partial n}\left|\nabla^{t} \tilde{\psi} \cdot n\right|^{2} \mathrm{~d} \sigma \mathrm{d} t+4 s^{3} \lambda^{4} \iint_{Q}\left|\nabla \eta^{0}\right|^{2} \tilde{\xi}^{3}\left|\tilde{\psi} \cdot \nabla \eta^{0}\right|^{2} \mathrm{~d} x \mathrm{~d} t \\
& +2 s \lambda^{2} \iint_{Q}\left|\nabla \eta^{0}\right|^{2} \tilde{\xi}|\nabla \tilde{\psi}|^{2} \mathrm{~d} x \mathrm{~d} t+2 s^{3} \lambda^{4} \iint_{Q}\left|\nabla \eta^{0}\right|^{4} \tilde{\xi}^{3}|\tilde{\psi}|^{2} \mathrm{~d} x \mathrm{~d} t \\
& -4 s \lambda^{2} \iint_{Q} \tilde{\xi}\left|\nabla^{t} \tilde{\psi} \nabla \eta^{0}\right|^{2} \mathrm{~d} x \mathrm{~d} t-12 s^{2} \lambda^{2} \iint_{Q} \tilde{\xi}^{2}\left|\nabla^{t} \tilde{\psi} \nabla \eta^{0}\right|^{2} \mathrm{~d} x \mathrm{~d} t \\
& +2\left(\left(M_{3} \tilde{\psi}\right)_{2},\left(M_{4} \tilde{\psi}\right)_{3}\right)_{L^{2}(Q)^{N}}+2\left(\left(M_{3} \tilde{\psi}\right)_{4},\left(M_{4} \tilde{\psi}\right)_{3}\right)_{L^{2}(Q)^{N}} \\
& -C\left(s \lambda \iint_{Q} \tilde{\xi}|\nabla \tilde{\psi}|^{2} \mathrm{~d} x \mathrm{~d} t+s \lambda^{3}\left(s+s^{2}+\lambda+s \lambda\right) \iint_{Q} \tilde{\xi}^{3}|\tilde{\psi}|^{2} \mathrm{~d} x \mathrm{~d} t\right. \\
& +s \lambda T^{7} \mathrm{e}^{\lambda\left\|\eta^{0}\right\|_{\infty}}\left(s \lambda+(1+\lambda) T^{8} \mathrm{e}^{\lambda\left\|\eta^{0}\right\|_{\infty}}\right) \iint_{Q} \tilde{\xi}^{3}|\tilde{\psi}|^{2} \mathrm{~d} x \mathrm{~d} t \\
& \left.+s T^{7} \mathrm{e}^{4 \lambda\left\|\eta^{0}\right\|_{\infty}}\left(T^{7} \mathrm{e}^{\lambda\left\|\eta^{0}\right\|_{\infty}}+s \lambda+s \lambda^{2}\right) \iint_{Q} \tilde{\xi}^{3}|\tilde{\psi}|^{2} \mathrm{~d} x \mathrm{~d} t\right) .
\end{aligned}
$$

Working as in the case of $\psi$, we find

$$
s^{3} \lambda^{4} \iint_{Q}\left|\nabla \eta^{0}\right|^{4} \tilde{\xi}^{3}|\tilde{\psi}|^{2} \mathrm{~d} x \mathrm{~d} t \geq C s^{3} \lambda^{4}\left(\iint_{Q} \tilde{\xi}^{3}|\tilde{\psi}|^{2} \mathrm{~d} x \mathrm{~d} t-\iint_{\omega^{\prime} \times(0, T)} \tilde{\xi}^{3}|\tilde{\psi}|^{2} \mathrm{~d} x \mathrm{~d} t\right)
$$

and

$$
s \lambda^{2} \iint_{Q}\left|\nabla \eta^{0}\right|^{2} \tilde{\xi}|\nabla \tilde{\psi}|^{2} \mathrm{~d} x \mathrm{~d} t \geq C s \lambda^{2}\left(\iint_{Q} \tilde{\xi}|\nabla \tilde{\psi}|^{2} \mathrm{~d} x \mathrm{~d} t-\iint_{\omega^{\prime} \times(0, T)} \tilde{\xi}|\nabla \tilde{\psi}|^{2} \mathrm{~d} x \mathrm{~d} t\right) .
$$

Making now the choice

$$
\lambda \geq C\left(\Omega, \omega^{\prime}\right), \quad s \geq C \mathrm{e}^{4 \lambda\left\|\eta^{0}\right\|_{\infty}}\left(T^{7}+T^{8}\right),
$$


we have

$$
\begin{aligned}
& C\left(s \lambda \iint_{Q} \xi|\nabla \psi|^{2} \mathrm{~d} x \mathrm{~d} t+s \lambda^{3}\left(s+s^{2}+\lambda+s \lambda\right) \iint_{Q} \xi^{3}|\psi|^{2} \mathrm{~d} x \mathrm{~d} t\right. \\
& +s \lambda T^{7} \mathrm{e}^{\lambda\left\|\eta^{0}\right\|_{\infty}}\left(s \lambda+(1+\lambda) T^{8} \mathrm{e}^{\lambda\left\|\eta^{0}\right\|_{\infty}}\right) \iint_{Q} \tilde{\xi}^{3}|\tilde{\psi}|^{2} \mathrm{~d} x \mathrm{~d} t \\
& \left.+s T^{7} \mathrm{e}^{4 \lambda\left\|\eta^{0}\right\|_{\infty}}\left(T^{7} \mathrm{e}^{\lambda\left\|\eta^{0}\right\|_{\infty}}+s \lambda+s \lambda^{2}\right) \iint_{Q} \tilde{\xi}^{3}|\tilde{\psi}|^{2} \mathrm{~d} x \mathrm{~d} t\right) \\
& \leq \tilde{\delta}\left(s^{3} \lambda^{4} \iint_{Q} \tilde{\xi}^{3}|\tilde{\psi}|^{2} \mathrm{~d} x \mathrm{~d} t+s \lambda^{2} \iint_{Q} \tilde{\xi}|\nabla \tilde{\psi}|^{2} \mathrm{~d} x \mathrm{~d} t\right)
\end{aligned}
$$

for a constant $\tilde{\delta}(\Omega, \omega)>0$ small enough.

Consequently, we get the following from (68)

$$
\begin{aligned}
& 2\left(M_{3} \tilde{\psi}, M_{4} \tilde{\psi}\right)_{L^{2}(Q)^{N}} \geq 2 \iint_{\Sigma}\left(\left(\nabla \tilde{\psi}+\nabla^{t} \tilde{\psi}\right) \cdot n\right)_{t g} \cdot \tilde{\psi}_{t} \mathrm{~d} \sigma \mathrm{d} t \\
& +4 s \lambda \iint_{\Sigma} \tilde{\xi} \frac{\partial \eta^{0}}{\partial n}\left(\left(\nabla \tilde{\psi}+\nabla^{t} \tilde{\psi}\right) \cdot n\right) \cdot(\nabla \tilde{\psi} \cdot n) \mathrm{d} \sigma \mathrm{d} t \\
& -2 s \lambda \iint_{\Sigma} \tilde{\xi} \frac{\partial \eta^{0}}{\partial n}|\nabla \tilde{\psi}|^{2} \mathrm{~d} \sigma \mathrm{d} t-4 s \lambda \iint_{\Sigma} \tilde{\xi} \frac{\partial \eta^{0}}{\partial n} \nabla \tilde{\psi}: \nabla^{t} \tilde{\psi} \mathrm{d} \sigma \mathrm{d} t \\
& +4 s \lambda \iint_{\Sigma} \tilde{\xi} \frac{\partial \eta^{0}}{\partial n}(\nabla \psi \cdot n) \cdot\left(\nabla^{t} \tilde{\psi} \cdot n\right) \mathrm{d} \sigma \mathrm{d} t \\
& +4 s \lambda \iint_{\Sigma} \tilde{\xi} \frac{\partial \eta^{0}}{\partial n}\left(\left(\nabla \tilde{\psi}+\nabla^{t} \tilde{\psi}\right) \cdot n\right) \cdot\left(\nabla^{t} \tilde{\psi} \cdot n\right) \mathrm{d} \sigma \mathrm{d} t \\
& -4 s \lambda^{2} \iint_{\Sigma} \tilde{\xi}\left|\frac{\partial \eta^{0}}{\partial n}\right|^{2}\left(\left(\nabla \tilde{\psi}+\nabla^{t} \tilde{\psi}\right) \cdot n\right)_{t g} \cdot \tilde{\psi} \mathrm{d} \sigma \mathrm{d} t \\
& +2 s^{3} \lambda^{3} \iint_{\Sigma} \tilde{\xi}^{3}\left(\frac{\partial \eta^{0}}{\partial n}\right)^{3}|\tilde{\psi}|^{2} \mathrm{~d} \sigma \mathrm{d} t+2 s^{2} \lambda \iint_{\Sigma} \frac{\partial \eta^{0}}{\partial n} \tilde{\xi} \tilde{\alpha}_{t}|\tilde{\psi}|^{2} \mathrm{~d} \sigma \mathrm{d} t \\
& -8 s \lambda \iint_{\Sigma} \tilde{\xi} \frac{\partial \eta^{0}}{\partial n}\left|\nabla^{t} \tilde{\psi} \cdot n\right|^{2} \mathrm{~d} \sigma \mathrm{d} t+4 s^{3} \lambda^{4} \iint_{Q}\left|\nabla \eta^{0}\right|^{2} \tilde{\xi}^{3}\left|\tilde{\psi} \cdot \nabla \eta^{0}\right|^{2} \mathrm{~d} x \mathrm{~d} t \\
& +s \lambda^{2} \iint_{Q} \tilde{\xi}|\nabla \tilde{\psi}|^{2} \mathrm{~d} x \mathrm{~d} t+s^{3} \lambda^{4} \iint_{Q} \tilde{\xi}^{3}|\tilde{\psi}|^{2} \mathrm{~d} x \mathrm{~d} t \\
& -4 s \lambda^{2} \iint_{Q} \tilde{\xi}\left|\nabla^{t} \tilde{\psi} \nabla \eta^{0}\right|^{2} \mathrm{~d} x \mathrm{~d} t-12 s^{2} \lambda^{2} \iint_{Q} \tilde{\xi}^{2}\left|\nabla^{t} \tilde{\psi} \nabla \eta^{0}\right|^{2} \mathrm{~d} x \mathrm{~d} t \\
& +2\left(\left(M_{3} \tilde{\psi}\right)_{2},\left(M_{4} \tilde{\psi}\right)_{3}\right)_{L^{2}(Q)^{N}}+2\left(\left(M_{3} \tilde{\psi}\right)_{4},\left(M_{4} \tilde{\psi}\right)_{3}\right)_{L^{2}(Q)^{N}}
\end{aligned}
$$

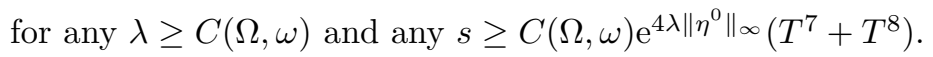

Let us also remark that

$$
\left\|\tilde{G}_{s, \lambda}\right\|_{L^{2}(Q)^{N}}^{2} \leq C\left(\iint_{Q} \mathrm{e}^{-2 s \tilde{\alpha}}|G|^{2} \mathrm{~d} x \mathrm{~d} t+s^{2} \lambda^{4} \iint_{Q} \tilde{\xi}^{2}|\tilde{\psi}|^{2} \mathrm{~d} x \mathrm{~d} t\right),
$$

for $\lambda \geq C(\Omega, \omega)$. 
As a conclusion, we obtain the following inequality up to now:

$$
\begin{aligned}
& \left\|M_{3} \tilde{\psi}\right\|_{L^{2}(Q)^{N}}^{2}+\left\|M_{4} \tilde{\psi}\right\|_{L^{2}(Q)^{N}}^{2}+s^{3} \lambda^{4} \iint_{Q} \tilde{\xi}^{3}|\tilde{\psi}|^{2} \mathrm{~d} x \mathrm{~d} t \\
& +s \lambda^{2} \iint_{Q} \tilde{\xi}|\nabla \tilde{\psi}|^{2} \mathrm{~d} x \mathrm{~d} t+4 s^{3} \lambda^{4} \iint_{Q}\left|\nabla \eta^{0}\right|^{2} \tilde{\xi}^{3}\left|\tilde{\psi} \cdot \nabla \eta^{0}\right|^{2} \mathrm{~d} x \mathrm{~d} t \\
& -4 s \lambda^{2} \iint_{Q} \tilde{\xi}\left|\nabla^{t} \tilde{\psi} \nabla \eta^{0}\right|^{2} \mathrm{~d} x \mathrm{~d} t-12 s^{2} \lambda^{2} \iint_{Q} \tilde{\xi}^{2}\left|\nabla^{t} \tilde{\psi} \nabla \eta^{0}\right|^{2} \mathrm{~d} x \mathrm{~d} t \\
& +2\left(\left(M_{3} \tilde{\psi}\right)_{2},\left(M_{4} \tilde{\psi}\right)_{3}\right)_{L^{2}(Q)^{N}}+2\left(\left(M_{3} \tilde{\psi}\right)_{4},\left(M_{4} \tilde{\psi}\right)_{3}\right)_{L^{2}(Q)^{N}} \\
& +2 \iint_{\Sigma}\left(\left(\nabla \tilde{\psi}+\nabla^{t} \tilde{\psi}\right) \cdot n\right)_{t g} \cdot \tilde{\psi}_{t} \mathrm{~d} \sigma \mathrm{d} t \\
& +4 s \lambda \iint_{\Sigma} \tilde{\xi} \frac{\partial \eta^{0}}{\partial n}\left(\left(\nabla \tilde{\psi}+\nabla^{t} \tilde{\psi}\right) \cdot n\right) \cdot(\nabla \tilde{\psi} \cdot n) \mathrm{d} \sigma \mathrm{d} t \\
& -2 s \lambda \iint_{\Sigma} \tilde{\xi} \frac{\partial \eta^{0}}{\partial n}|\nabla \tilde{\psi}|^{2} \mathrm{~d} \sigma \mathrm{d} t-4 s \lambda \iint_{\Sigma} \tilde{\xi} \frac{\partial \eta^{0}}{\partial n} \nabla \tilde{\psi}: \nabla^{t} \tilde{\psi} \mathrm{d} \sigma \mathrm{d} t \\
& +4 s \lambda \iint_{\Sigma} \tilde{\xi} \frac{\partial \eta^{0}}{\partial n}(\nabla \tilde{\psi} \cdot n) \cdot\left(\nabla^{t} \tilde{\psi} \cdot n\right) \mathrm{d} \sigma \mathrm{d} t \\
& +4 s \lambda \iint_{\Sigma} \tilde{\xi} \frac{\partial \eta^{0}}{\partial n}\left(\left(\nabla \tilde{\psi}+\nabla^{t} \tilde{\psi}\right) \cdot n\right) \cdot\left(\nabla^{t} \tilde{\psi} \cdot n\right) \mathrm{d} \sigma \mathrm{d} t \\
& -4 s \lambda^{2} \iint_{\Sigma} \tilde{\xi}\left|\frac{\partial \eta^{0}}{\partial n}\right|^{2}\left(\left(\nabla \tilde{\psi}+\nabla^{t} \tilde{\psi}\right) \cdot n\right)_{t g} \cdot \tilde{\psi} \mathrm{d} \sigma \mathrm{d} t \\
& +2 s^{3} \lambda^{3} \iint_{\Sigma} \tilde{\xi}^{3}\left(\frac{\partial \eta^{0}}{\partial n}\right)^{3}|\tilde{\psi}|^{2} \mathrm{~d} \sigma \mathrm{d} t+2 s^{2} \lambda \iint_{\Sigma} \frac{\partial \eta^{0}}{\partial n} \tilde{\xi} \tilde{\alpha}_{t}|\tilde{\psi}|^{2} \mathrm{~d} \sigma \mathrm{d} t \\
& -8 s \lambda \iint_{\Sigma} \tilde{\xi} \frac{\partial \eta^{0}}{\partial n}\left|\nabla^{t} \tilde{\psi} \cdot n\right|^{2} \mathrm{~d} \sigma \mathrm{d} t \\
& \leq C\left(\iint_{Q} \mathrm{e}^{-2 s \tilde{\alpha}}|G|^{2} \mathrm{~d} x \mathrm{~d} t+s^{3} \lambda^{4} \iint_{\omega^{\prime} \times(0, T)} \tilde{\xi}^{3}|\tilde{\psi}|^{2} \mathrm{~d} x \mathrm{~d} t\right. \\
& \left.+s \lambda^{2} \iint_{\omega^{\prime} \times(0, T)} \tilde{\xi}|\nabla \tilde{\psi}|^{2} \mathrm{~d} x \mathrm{~d} t\right),
\end{aligned}
$$

for any $\lambda \geq C(\Omega, \omega)$ and any $s \geq C(\Omega, \omega) \mathrm{e}^{4 \lambda\left\|\eta^{0}\right\|_{\infty}}\left(T^{7}+T^{8}\right)$.

Similar computations to those in step 1 must be made now for $M_{3} \tilde{\psi}$ and $M_{4} \tilde{\psi}$. As above, we just write all the integrals coming out from these calculations.

For $M_{3} \tilde{\psi}$, we have

$$
M_{3} \tilde{\psi}=\bar{M}_{3} \tilde{\psi}+2 s \lambda \tilde{\xi} \nabla^{t} \tilde{\psi} \cdot \nabla \eta^{0}
$$

with

$$
\bar{M}_{3} \tilde{\psi}=-\Delta \tilde{\psi}-s^{2} \lambda^{2}\left|\nabla \eta^{0}\right|^{2} \tilde{\xi}^{2} \tilde{\psi}-s \tilde{\alpha}_{t} \tilde{\psi}+s \lambda \tilde{\xi}\left(\nabla \nabla \eta^{0} \cdot \tilde{\psi}\right)-s \lambda^{2} \nabla \eta^{0} \tilde{\xi}\left(\tilde{\psi} \cdot \nabla \eta^{0}\right)
$$

Developing again the $L^{2}$ norm of $M_{3} \tilde{\psi}$, we focus on the double product term:

$$
\begin{aligned}
& 2\left(\bar{M}_{3} \tilde{\psi}, s \lambda \tilde{\xi} \nabla^{t} \tilde{\psi} \cdot \nabla \eta^{0}\right)_{L^{2}(Q)^{N}}=-2\left(\bar{M}_{3} \tilde{\psi},\left(M_{4} \tilde{\psi}\right)_{3}\right)_{L^{2}(Q)^{N}} \\
&=-2\left(\left(M_{3} \tilde{\psi}\right)_{2},\left(M_{4} \tilde{\psi}\right)_{3}\right)-2\left(\left(M_{3} \tilde{\psi}\right)_{4},\left(M_{4} \tilde{\psi}\right)_{3}\right)_{L^{2}(Q)^{N}}+4 \tilde{E}_{244}-2 \tilde{H}_{41} \\
&+\tilde{\mathcal{T}}_{1}+\tilde{\mathcal{T}}_{21}+\tilde{\mathcal{T}}_{22}+2 \tilde{\mathcal{T}}_{23}+\tilde{\mathcal{T}}_{31}+\tilde{\mathcal{T}}_{32}+\tilde{\mathcal{T}}_{331}
\end{aligned}
$$


where

$$
\begin{gathered}
\tilde{\mathcal{T}}_{1}=4 s \lambda \iint_{Q} \tilde{\xi}\left(\nabla \nabla \eta^{0} \cdot \tilde{\psi}\right) \cdot \Delta \tilde{\psi} \mathrm{d} x \mathrm{~d} t \\
\tilde{\mathcal{T}}_{21}=4 s \lambda^{2} \iint_{Q} \tilde{\xi}\left(\nabla\left(\nabla \eta^{0} \nabla \eta^{0}\right) \cdot \nabla \tilde{\psi}\right) \cdot \tilde{\psi} \mathrm{d} x \mathrm{~d} t, \\
\tilde{\mathcal{T}}_{22}=-4 s \lambda^{3} \iint_{Q} \tilde{\xi}\left(\tilde{\psi} \cdot \nabla \eta^{0}\right)\left(\nabla \tilde{\psi} \cdot \nabla \eta^{0}\right) \cdot \nabla \eta^{0} \mathrm{~d} x \mathrm{~d} t, \\
\tilde{\mathcal{T}}_{23}=4 s \lambda^{2} \iint_{Q} \tilde{\xi}\left|\nabla^{t} \tilde{\psi} \cdot \nabla \eta^{0}\right|^{2} \mathrm{~d} x \mathrm{~d} t \\
\tilde{\mathcal{T}}_{31}=4 s^{2} \lambda^{2} \iint_{Q} \tilde{\xi}\left(\nabla \nabla \eta^{0} \cdot \tilde{\psi}\right) \cdot \nabla\left(\tilde{\xi} \tilde{\psi} \cdot \nabla \eta^{0}\right) \mathrm{d} x \mathrm{~d} t \\
\tilde{\mathcal{T}}_{32}=-2 s^{2} \lambda^{3} \iint_{Q} \nabla \eta^{0} \cdot \nabla\left|\tilde{\xi} \tilde{\psi} \cdot \nabla \eta^{0}\right|^{2} \mathrm{~d} x \mathrm{~d} t
\end{gathered}
$$

and

$$
\tilde{T}_{331}=4 s^{2} \lambda^{2} \iint_{Q} \tilde{\xi}^{2}\left|\nabla^{t} \tilde{\psi} \cdot \nabla \eta^{0}\right|^{2} \mathrm{~d} x \mathrm{~d} t .
$$

Thus, from (70) we deduce the following inequality:

$$
\begin{aligned}
& \left\|\bar{M}_{3} \tilde{\psi}\right\|_{L^{2}(Q)^{N}}^{2}+\left\|M_{4} \tilde{\psi}\right\|_{L^{2}(Q)^{N}}^{2}+s^{3} \lambda^{4} \iint_{Q} \tilde{\xi}^{3}|\tilde{\psi}|^{2} \mathrm{~d} x \mathrm{~d} t \\
& +s \lambda^{2} \iint_{Q} \tilde{\xi}|\nabla \tilde{\psi}|^{2} \mathrm{~d} x \mathrm{~d} t+4 s^{3} \lambda^{4} \iint_{Q}\left|\nabla \eta^{0}\right|^{2} \tilde{\xi}^{3}\left|\tilde{\psi} \cdot \nabla \eta^{0}\right|^{2} \mathrm{~d} x \mathrm{~d} t \\
& -4 s^{2} \lambda^{2} \iint_{Q} \tilde{\xi}^{2}\left|\nabla^{t} \tilde{\psi} \nabla \eta^{0}\right|^{2} \mathrm{~d} x \mathrm{~d} t+2 \iint_{\Sigma}\left(\left(\nabla \tilde{\psi}+\nabla^{t} \tilde{\psi}\right) \cdot n\right)_{t g} \cdot \tilde{\psi}_{t} \mathrm{~d} \sigma \mathrm{d} t \\
& +4 s \lambda \iint_{\Sigma} \tilde{\xi} \frac{\partial \eta^{0}}{\partial n}\left(\left(\nabla \tilde{\psi}+\nabla^{t} \tilde{\psi}\right) \cdot n\right) \cdot(\nabla \tilde{\psi} \cdot n) \mathrm{d} \sigma \mathrm{d} t \\
& -2 s \lambda \iint_{\Sigma} \tilde{\xi} \frac{\partial \eta^{0}}{\partial n}|\nabla \tilde{\psi}|^{2} \mathrm{~d} \sigma \mathrm{d} t-4 s \lambda \iint_{\Sigma} \tilde{\xi} \frac{\partial \eta^{0}}{\partial n} \nabla \tilde{\psi}: \nabla^{t} \tilde{\psi} \mathrm{d} \sigma \mathrm{d} t \\
& +4 s \lambda \iint_{\Sigma} \tilde{\xi} \frac{\partial \eta^{0}}{\partial n}(\nabla \tilde{\psi} \cdot n) \cdot\left(\nabla^{t} \tilde{\psi} \cdot n\right) \mathrm{d} \sigma \mathrm{d} t \\
& +4 s \lambda \iint_{\Sigma} \tilde{\xi} \frac{\partial \eta^{0}}{\partial n}\left(\left(\nabla \tilde{\psi}+\nabla^{t} \tilde{\psi}\right) \cdot n\right) \cdot\left(\nabla^{t} \tilde{\psi} \cdot n\right) \mathrm{d} \sigma \mathrm{d} t \\
& -4 s \lambda^{2} \iint_{\Sigma} \tilde{\xi}\left|\frac{\partial \eta^{0}}{\partial n}\right|^{2}\left(\left(\nabla \tilde{\psi}+\nabla^{t} \tilde{\psi}\right) \cdot n\right)_{t g} \cdot \tilde{\psi} \mathrm{d} \sigma \mathrm{d} t \\
& +2 s^{3} \lambda^{3} \iint_{\Sigma} \tilde{\xi}^{3}\left(\frac{\partial \eta^{0}}{\partial n}\right)^{3}|\tilde{\psi}|^{2} \mathrm{~d} \sigma \mathrm{d} t+2 s^{2} \lambda \iint_{\Sigma} \frac{\partial \eta^{0}}{\partial n} \tilde{\xi} \tilde{\alpha}_{t}|\tilde{\psi}|^{2} \mathrm{~d} \sigma \mathrm{d} t \\
& -8 s \lambda \iint_{\Sigma} \tilde{\xi} \frac{\partial \eta^{0}}{\partial n}\left|\nabla^{t} \tilde{\psi} \cdot n\right|^{2} \mathrm{~d} \sigma \mathrm{d} t \\
& \leq C\left(\iint_{Q} \mathrm{e}^{-2 s \tilde{\alpha}}|G|^{2} \mathrm{~d} x \mathrm{~d} t+s \lambda \iint_{Q} \tilde{\xi}|\tilde{\psi}||\Delta \tilde{\psi}| \mathrm{d} x \mathrm{~d} t\right. \\
& \left.+s^{3} \lambda^{4} \iint_{\omega^{\prime} \times(0, T)} \tilde{\xi}^{3}|\tilde{\psi}|^{2} \mathrm{~d} x \mathrm{~d} t+s \lambda^{2} \iint_{\omega^{\prime} \times(0, T)} \tilde{\xi}|\nabla \tilde{\psi}|^{2} \mathrm{~d} x \mathrm{~d} t\right),
\end{aligned}
$$

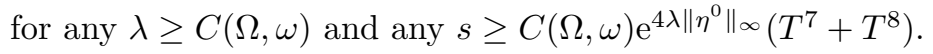


On the other hand, for $M_{4} \tilde{\psi}$, we have:

$$
M_{4} \tilde{\psi}=\bar{M}_{4} \tilde{\psi}-2 s \lambda \tilde{\xi} \nabla^{t} \tilde{\psi} \cdot \nabla \eta^{0}
$$

with

$$
\bar{M}_{4} \tilde{\psi}=\left(M_{4} \tilde{\psi}\right)_{1}+\left(M_{4} \tilde{\psi}\right)_{2}+\left(M_{4} \tilde{\psi}\right)_{4}
$$

Employing now similar estimates as in the first case, we find

$$
\begin{aligned}
& \left\|M_{4} \tilde{\psi}\right\|_{L^{2}(Q)^{N}}^{2} \geq 4 s^{2} \lambda^{2} \iint_{Q} \tilde{\xi}^{2}\left|\nabla^{t} \tilde{\psi} \cdot \nabla \eta^{0}\right|^{2} \mathrm{~d} x \mathrm{~d} t+\left\|\bar{M}_{4} \tilde{\psi}\right\|_{L^{2}(Q)^{N}}^{2} \\
& \quad-4 s^{3} \lambda^{4} \iint_{Q}\left|\nabla \eta^{0}\right|^{2} \tilde{\xi}^{3}\left|\tilde{\psi} \cdot \nabla \eta^{0}\right|^{2} \mathrm{~d} x \mathrm{~d} t \\
& \quad-C\left(s \lambda T(1+\lambda) \mathrm{e}^{(\lambda / 4)\left\|\eta^{0}\right\|} \iint_{Q} \tilde{\xi}^{5 / 4}|\tilde{\psi}|^{2} \mathrm{~d} x \mathrm{~d} t+s \lambda \iint_{Q} \tilde{\xi}|\nabla \tilde{\psi}|^{2} \mathrm{~d} x \mathrm{~d} t\right. \\
& \left.+s^{3} \lambda^{3} \iint_{Q} \tilde{\xi}^{3}|\tilde{\psi}|^{2} \mathrm{~d} x \mathrm{~d} t+s^{2} \lambda^{3}(1+\lambda) \iint_{Q} \tilde{\xi}^{2}|\tilde{\psi}|^{2} \mathrm{~d} x \mathrm{~d} t\right) .
\end{aligned}
$$

This, together with (71), provides the estimate we were looking for in this second step:

$$
\begin{aligned}
& \left\|\bar{M}_{3} \tilde{\psi}\right\|_{L^{2}(Q)^{N}}^{2}+\| \bar{M}_{4} \tilde{\psi}_{L^{2}(Q)^{N}}^{2}+s^{3} \lambda^{4} \iint_{Q} \tilde{\xi}^{3}|\tilde{\psi}|^{2} \mathrm{~d} x \mathrm{~d} t \\
& +s \lambda^{2} \iint_{Q} \tilde{\xi}|\nabla \tilde{\psi}|^{2} \mathrm{~d} x \mathrm{~d} t+2 \iint_{\Sigma}\left(\left(\nabla \tilde{\psi}+\nabla^{t} \tilde{\psi}\right) \cdot n\right)_{t g} \cdot \psi_{t} \mathrm{~d} \sigma \mathrm{d} t \\
& +4 s \lambda \iint_{\Sigma} \tilde{\xi} \frac{\partial \eta^{0}}{\partial n}\left(\left(\nabla \tilde{\psi}+\nabla^{t} \tilde{\psi}\right) \cdot n\right) \cdot(\nabla \tilde{\psi} \cdot n) \mathrm{d} \sigma \mathrm{d} t \\
& -2 s \lambda \iint_{\Sigma} \tilde{\xi} \frac{\partial \eta^{0}}{\partial n}|\nabla \tilde{\psi}|^{2} \mathrm{~d} \sigma \mathrm{d} t-4 s \lambda \iint_{\Sigma} \tilde{\xi} \frac{\partial \eta^{0}}{\partial n} \nabla \tilde{\psi}: \nabla^{t} \tilde{\psi} \mathrm{d} \sigma \mathrm{d} t \\
& +4 s \lambda \iint_{\Sigma} \tilde{\xi} \frac{\partial \eta^{0}}{\partial n}(\nabla \tilde{\psi} \cdot n) \cdot\left(\nabla^{t} \tilde{\psi} \cdot n\right) \mathrm{d} \sigma \mathrm{d} t \\
& +4 s \lambda \iint_{\Sigma} \tilde{\xi} \frac{\partial \eta^{0}}{\partial n}\left(\left(\nabla \tilde{\psi}+\nabla^{t} \tilde{\psi}\right) \cdot n\right) \cdot\left(\nabla^{t} \tilde{\psi} \cdot n\right) \mathrm{d} \sigma \mathrm{d} t \\
& -4 s \lambda^{2} \iint_{\Sigma} \tilde{\xi}\left|\frac{\partial \eta^{0}}{\partial n}\right|^{2}\left(\left(\nabla \tilde{\psi}+\nabla^{t} \tilde{\psi}\right) \cdot n\right)_{t g} \cdot \tilde{\psi} \mathrm{d} \sigma \mathrm{d} t \\
& +2 s^{3} \lambda^{3} \iint_{\Sigma} \tilde{\xi}^{3}\left(\frac{\partial \eta^{0}}{\partial n}\right)^{3}|\tilde{\psi}|^{2} \mathrm{~d} \sigma \mathrm{d} t+2 s^{2} \lambda \iint_{\Sigma} \frac{\partial \eta^{0}}{\partial n} \tilde{\xi} \tilde{\alpha}_{t}|\tilde{\psi}|^{2} \mathrm{~d} \sigma \mathrm{d} t \\
& -8 s \lambda \iint_{\Sigma} \tilde{\xi} \frac{\partial \eta^{0}}{\partial n}\left|\nabla^{t} \tilde{\psi} \cdot n\right|^{2} \mathrm{~d} \sigma \mathrm{d} t \\
& \leq C\left(\iint_{Q} \mathrm{e}^{-2 s \tilde{\alpha}}|G|^{2} \mathrm{~d} x \mathrm{~d} t+s \lambda \iint_{Q} \tilde{\xi}|\tilde{\psi} \| \Delta \tilde{\psi}| \mathrm{d} x \mathrm{~d} t\right. \\
& \left.+s^{3} \lambda^{4} \iint_{\omega^{\prime} \times(0, T)} \tilde{\xi}^{3}|\tilde{\psi}|^{2} \mathrm{~d} x \mathrm{~d} t+s \lambda^{2} \iint_{\omega^{\prime} \times(0, T)} \tilde{\xi}|\nabla \tilde{\psi}|^{2} \mathrm{~d} x \mathrm{~d} t\right),
\end{aligned}
$$

for any $\lambda \geq C(\Omega, \omega)$ and any $s \geq C(\Omega, \omega) \mathrm{e}^{4 \lambda\left\|\eta^{0}\right\|_{\infty}}\left(T^{7}+T^{8}\right)$. 
Step 3. Last arrangements and conclusion.

In this paragraph we will combine the inequalities obtained in steps 1 and $2((61)$ and (72)). Firstly, we deal with the integrals on the boundary. Then, we will eliminate the local term of $\nabla \psi$ (and $\nabla \tilde{\psi}$ ). Finally, we will turn back to the original variable $\varphi$ and we will deduce the inequality (47).

Let us thus study the terms on $\Sigma$. To this end, we set some relations which will be useful in the sequel, namely:

$$
\left.\begin{array}{l}
\nabla \psi^{i}=\mathrm{e}^{-s \alpha}\left(\nabla \varphi^{i}+s \lambda \nabla \eta^{0} \xi \varphi^{i}\right) \\
\nabla \tilde{\psi}^{i}=\mathrm{e}^{-s \alpha}\left(\nabla \varphi^{i}-s \lambda \nabla \eta^{0} \xi \varphi^{i}\right)
\end{array}\right\} \text { on } \Sigma \text {, for } i=1, \ldots, N .
$$

These come directly from the fact that

$$
\xi=\tilde{\xi}, \quad \alpha=\tilde{\alpha} \quad \text { and } \quad \psi=\tilde{\psi} \quad \text { on } \Sigma .
$$

Consequently, since we have $\varphi \cdot n=0$ on $\Sigma$, we obtain

$$
\begin{cases}\nabla \psi \cdot n=\mathrm{e}^{-s \alpha}\left(\nabla \varphi \cdot n+s \lambda \frac{\partial \eta^{0}}{\partial n} \xi \varphi\right), & \nabla^{t} \psi \cdot n=\mathrm{e}^{-s \alpha}\left(\nabla^{t} \varphi \cdot n\right) \\ \nabla \tilde{\psi} \cdot n=\mathrm{e}^{-s \alpha}\left(\nabla \varphi \cdot n-s \lambda \frac{\partial \eta^{0}}{\partial n} \xi \varphi\right), & \nabla^{t} \tilde{\psi} \cdot n=\mathrm{e}^{-s \alpha}\left(\nabla^{t} \varphi \cdot n\right) .\end{cases}
$$

Let us start computing $2 A_{1}+2 \tilde{A}_{1}$ :

$$
\begin{aligned}
2 A_{1}+2 \tilde{A}_{1}= & 2 \iint_{\Sigma}\left(\left(\nabla \psi+\nabla^{t} \psi\right) \cdot n\right)_{t g} \cdot \psi_{t} \mathrm{~d} \sigma \mathrm{d} t \\
& +2 \iint_{\Sigma}\left(\left(\nabla \tilde{\psi}+\nabla^{t} \tilde{\psi}\right) \cdot n\right)_{t g} \cdot \tilde{\psi}_{t} \mathrm{~d} \sigma \mathrm{d} t \\
= & 2 \iint_{\Sigma} \mathrm{e}^{-s \alpha}\left(\nabla \varphi \cdot n+\nabla^{t} \varphi \cdot n+s \lambda \frac{\partial \eta^{0}}{\partial n} \xi \varphi\right)_{t g} \cdot\left(\mathrm{e}^{-s \alpha} \varphi\right)_{t} \mathrm{~d} \sigma \mathrm{d} t \\
& +2 \iint_{\Sigma} \mathrm{e}^{-s \alpha}\left(\nabla \varphi \cdot n+\nabla^{t} \varphi \cdot n-s \lambda \frac{\partial \eta^{0}}{\partial n} \xi \varphi\right)_{t g} \cdot\left(\mathrm{e}^{-s \alpha} \varphi\right)_{t} \mathrm{~d} \sigma \mathrm{d} t \\
= & 2 \iint_{\Sigma} \mathrm{e}^{-s \alpha}(A \varphi) \cdot\left(\mathrm{e}^{-s \alpha} \varphi\right)_{t} \mathrm{~d} \sigma \mathrm{d} t \\
\leq & C\|A\|_{H^{(1-\ell) / 2}\left(H^{-3 / 2+N / 2+2 \ell)}\right.}\left\|\mathrm{e}^{-s \alpha^{*}} \varphi\right\|_{H^{(1+\ell) / 2}\left(H^{1 / 2-\ell}\right)}^{2}
\end{aligned}
$$

To prove the last estimate, it suffices to realize that the product of two functions, one of them $\left(\mathrm{e}^{-s \alpha^{*}} \varphi\right)$ belonging to the space $H^{(1+\ell) / 2}\left(0, T ; H^{1 / 2-\ell}(\partial \Omega)^{N}\right)$ and the other one $(A)$ belonging to $H^{(1-\ell) / 2}\left(0, T ; H^{-3 / 2+N / 2+2 \ell}(\partial \Omega)^{N^{2}}\right)$, is actually in

$$
H^{(1-\ell) / 2}\left(0, T ; H^{-1 / 2+\ell}(\partial \Omega)\right) \text {. }
$$

Here, we have used the hypothesis (9) (observe that (9) implies that the previous norm of $A$ is finite) and the fact that $\alpha_{\mid \Sigma}(x, t)=\alpha^{*}(t)$ (see (44) above).

In order to estimate the last expression, let us introduce the function $\varphi^{*}=\mathrm{e}^{-s \alpha^{*}} \varphi$. It verifies:

$$
\begin{cases}-\varphi_{t}^{*}-\nabla \cdot\left(D \varphi^{*}\right)=G^{*} & \text { in } Q \\ \varphi^{*} \cdot n=0, \quad\left(D \varphi^{*} \cdot n\right)_{t g}+\left(A(x, t) \varphi^{*}\right)_{t g}=0 & \text { on } \Sigma \\ \varphi^{*}(\cdot, T)=0 & \text { in } \Omega\end{cases}
$$

with

$$
G^{*}=\mathrm{e}^{-s \alpha^{*}} G-\left(\mathrm{e}^{-s \alpha^{*}}\right)_{t} \varphi
$$


For this system, one can perform a similar proof to that of Proposition 1.1 (using Lax-Milgram's lemma instead of a mixed problem) and obtain a the corresponding estimate equivalent to (18). Besides, the interpolation inequality (36) tells us that the term

$$
\left\|\varphi^{*}\right\|_{H^{(1+\ell) / 2}\left(H^{1 / 2-\ell}\right)}^{2}
$$

can be estimated in terms of its norm as a strong solution (i.e., $\varphi_{t}^{*} \in L^{2}$ and $\left.\varphi^{*} \in L^{2}\left(H^{2}\right)\right)$. More precisely, we have

$$
\left\|\varphi^{*}\right\|_{H^{(1+\ell) / 2}\left(H^{1 / 2-\ell}\right)}^{2} \leq C\left\|\varphi_{t}^{*}\right\|_{L^{2}(Q)^{N}}^{1+\ell}\left\|\varphi^{*}\right\|_{L^{2}\left(H^{2}\right)}^{1-\ell} \leq C\left(\left\|\varphi_{t}^{*}\right\|_{L^{2}(Q)^{N}}^{2}+\left\|\varphi^{*}\right\|_{L^{2}\left(H^{2}\right)}^{2}\right) .
$$

Let us now use estimate (18). This yields

$$
\left\|\mathrm{e}^{-s \alpha^{*}} \varphi\right\|_{H^{(1+\ell) / 2}\left(H^{1 / 2-\ell}\right)}^{2} \leq C \mathrm{e}^{C T\|A\|_{P}^{2}}\left(1+\|A\|_{P}^{4}\right)\left(\left\|\mathrm{e}^{-s \alpha^{*}} G\right\|_{L^{2}(Q)^{N}}^{2}+\left\|\left(\mathrm{e}^{-s \alpha^{*}}\right)_{t} \varphi\right\|_{L^{2}(Q)^{N}}^{2}\right) .
$$

Similarly to (55), we find

$$
\left|\alpha_{t}^{*}\right| \leq C T \mathrm{e}^{2 \lambda\left\|\eta^{0}\right\|_{\infty}}\left(\xi^{*}\right)^{5 / 4}
$$

Consequently, we obtain from (73)

$$
2 A_{1}+2 \tilde{A}_{1} \leq \mathrm{e}^{C T\|A\|_{P}^{2}}\left(1+\|A\|_{P}^{5}\right)\left(\iint_{Q} \mathrm{e}^{-2 s \alpha}|G|^{2} \mathrm{~d} x \mathrm{~d} t+s^{2} \mathrm{e}^{4 \lambda\left\|\eta^{0}\right\| \infty} T^{2} \iint_{Q} \mathrm{e}^{-2 s \alpha} \xi^{5 / 2}|\varphi|^{2} \mathrm{~d} x \mathrm{~d} t\right) .
$$

Choosing $\lambda \geq C \mathrm{e}^{C T\|A\|_{P}^{2}}\left(1+\|A\|_{P}^{5}\right)$ and $s \geq C \mathrm{e}^{4 \lambda\left\|\eta^{0}\right\|}\left(T^{6}+T^{8}\right)$, we will be able to absorb the last term, while the first one is bounded by

$$
\lambda \iint_{Q} \mathrm{e}^{-2 s \alpha}|G|^{2} \mathrm{~d} x \mathrm{~d} t
$$

Additionally,

$$
\begin{aligned}
2 B+2 F_{1}+2 \tilde{B}+2 \tilde{F}_{1}= & -4 s \lambda \iint_{\Sigma} \xi \frac{\partial \eta^{0}}{\partial n}\left|\nabla \psi \cdot n+\nabla^{t} \psi \cdot n\right|^{2} \mathrm{~d} \sigma \mathrm{d} t \\
& +4 s \lambda \iint_{\Sigma} \xi \frac{\partial \eta^{0}}{\partial n}|\nabla \tilde{\psi} \cdot n+\nabla \tilde{\psi} \cdot n|^{2} \mathrm{~d} \sigma \mathrm{d} t \\
= & -16 s^{2} \lambda^{2} \iint_{\Sigma} \mathrm{e}^{-2 s \alpha}\left|\frac{\partial \eta^{0}}{\partial n}\right|^{2} \xi^{2}\left(\nabla \varphi \cdot n+\nabla^{t} \varphi \cdot n\right)_{t g} \cdot \varphi \mathrm{d} \sigma \mathrm{d} t .
\end{aligned}
$$

Moreover,

$$
\begin{aligned}
2 E_{11}+2 \tilde{E}_{11} & =2 s \lambda \iint_{\Sigma} \xi \frac{\partial \eta^{0}}{\partial n}|\nabla \psi|^{2} \mathrm{~d} \sigma \mathrm{d} t-2 s \lambda \iint_{\Sigma} \xi \frac{\partial \eta^{0}}{\partial n}|\nabla \tilde{\psi}|^{2} \mathrm{~d} x \mathrm{~d} t \\
& =8 s^{2} \lambda^{2} \iint_{\Sigma} \mathrm{e}^{-2 s \alpha}\left|\frac{\partial \eta^{0}}{\partial n}\right|^{2} \xi^{2}(\nabla \varphi \cdot n)_{t g} \cdot \varphi \mathrm{d} \sigma \mathrm{d} t .
\end{aligned}
$$

Besides,

$$
\begin{aligned}
2 E_{21}+2 \tilde{E}_{21} & =4 s \lambda \iint_{\Sigma} \xi \frac{\partial \eta^{0}}{\partial n} \nabla \psi: \nabla^{t} \psi \mathrm{d} \sigma \mathrm{d} t-4 s \lambda \iint_{\Sigma} \xi \frac{\partial \eta^{0}}{\partial n} \nabla \tilde{\psi}: \nabla^{t} \tilde{\psi} \mathrm{d} \sigma \mathrm{d} t \\
& =16 s^{2} \lambda^{2} \iint_{\Sigma} \mathrm{e}^{-2 s \alpha} \xi^{2}\left|\frac{\partial \eta^{0}}{\partial n}\right|^{2}\left(\nabla^{t} \varphi \cdot n\right)_{t g} \cdot \varphi \mathrm{d} \sigma \mathrm{d} t
\end{aligned}
$$


Then,

$$
\begin{aligned}
2 E_{241}+2 \tilde{E}_{241}= & -4 s \lambda \iint_{\Sigma} \xi \frac{\partial \eta^{0}}{\partial n}(\nabla \psi \cdot n) \cdot\left(\nabla^{t} \psi \cdot n\right) \mathrm{d} \sigma \mathrm{d} t \\
& +4 s \lambda \iint_{\Sigma} \xi \frac{\partial \eta^{0}}{\partial n}(\nabla \tilde{\psi} \cdot n) \cdot\left(\nabla^{t} \tilde{\psi} \cdot n\right) \mathrm{d} \sigma \mathrm{d} t \\
= & -8 s^{2} \lambda^{2} \iint_{\Sigma} \mathrm{e}^{-2 s \alpha} \xi^{2}\left|\frac{\partial \eta^{0}}{\partial n}\right|^{2}\left(\nabla^{t} \varphi \cdot n\right)_{t g} \cdot \varphi \mathrm{d} \sigma \mathrm{d} t .
\end{aligned}
$$

For $I_{1}$ and $\tilde{I}_{1}$, we have

$$
\begin{aligned}
2 I_{1}+2 \tilde{I}_{1}= & -4 s \lambda^{2} \iint_{\Sigma} \xi\left|\frac{\partial \eta^{0}}{\partial n}\right|^{2}\left(\left(\nabla \psi+\nabla^{t} \psi\right) \cdot n\right)_{t g} \cdot \psi \mathrm{d} \sigma \mathrm{d} t \\
& -4 s \lambda^{2} \iint_{\Sigma} \xi\left|\frac{\partial \eta^{0}}{\partial n}\right|^{2}\left(\left(\nabla \tilde{\psi}+\nabla^{t} \tilde{\psi}\right) \cdot n\right)_{t g} \cdot \tilde{\psi} \mathrm{d} \sigma \mathrm{d} t \\
= & 8 s \lambda^{2} \iint_{\Sigma} \mathrm{e}^{-2 s \alpha} \xi\left|\frac{\partial \eta^{0}}{\partial n}\right|^{2}(A \varphi)_{t g} \cdot \varphi \mathrm{d} \sigma \mathrm{d} t .
\end{aligned}
$$

Finally, we observe that

$$
H_{1}=-\tilde{H}_{1}, \quad K_{1}=-\tilde{K}_{1} \quad \text { and } \quad R_{1}=-\tilde{R}_{1}
$$

As a conclusion, an addition of the boundary terms in (61) and (72) yields an expression that can be bounded as follows:

$$
C\left(s^{2} \lambda^{2}\|A\|_{\infty}^{2} \iint_{\Sigma} \mathrm{e}^{-2 s \alpha} \xi^{2}|\varphi|^{2} \mathrm{~d} \sigma \mathrm{d} t+\lambda \iint_{Q} \mathrm{e}^{-2 s \alpha}|G|^{2} \mathrm{~d} x \mathrm{~d} t\right)+\varepsilon s^{3} \lambda^{4} \iint_{Q} \mathrm{e}^{-2 s \alpha} \xi^{3}|\varphi|^{2} \mathrm{~d} x \mathrm{~d} t
$$

for a constant $\varepsilon=\varepsilon(\Omega, \omega)>0$ small enough and where we have taken $\lambda \geq C \mathrm{e}^{C T\|A\|_{P}^{2}}\left(1+\|A\|_{P}^{5}\right)$ and $s \geq$ $C \mathrm{e}^{4 \lambda\left\|\eta^{0}\right\|}\left(T^{6}+T^{8}\right)$.

A simple computation proves that the first term can also be absorbed. In fact,

$-s^{2} \lambda^{2} \iint_{Q} \mathrm{e}^{-2 s \alpha} \xi^{2} \nabla \eta^{0} \cdot \nabla|\varphi|^{2} \mathrm{~d} x \mathrm{~d} t=s^{2} \lambda^{2} \iint_{\Sigma} \mathrm{e}^{-2 s \alpha} \xi^{2}\left|\frac{\partial \eta^{0}}{\partial n}\right||\varphi|^{2} \mathrm{~d} \sigma \mathrm{d} t$

$$
+s^{2} \lambda^{2} \iint_{Q} \nabla\left(\mathrm{e}^{-2 s \alpha} \xi^{2}\right) \cdot \nabla \eta^{0}|\varphi|^{2} \mathrm{~d} x \mathrm{~d} t
$$

so

$$
s^{2} \lambda^{2} \iint_{\Sigma} \mathrm{e}^{-2 s \alpha} \xi^{2}|\varphi|^{2} \mathrm{~d} \sigma \mathrm{d} t \leq C\left(s^{3} \lambda^{3} \int_{Q} \mathrm{e}^{-2 s \alpha} \xi^{3}|\varphi|^{2} \mathrm{~d} x \mathrm{~d} t+s \lambda \iint_{Q} \mathrm{e}^{-2 s \alpha} \xi|\nabla \varphi|^{2} \mathrm{~d} x \mathrm{~d} t\right),
$$

for $s \geq C T^{8}$ and $\lambda \geq C$. 
Consequently, if we take $s \geq C T^{8}$ and $\lambda \geq C\left(1+\|A\|_{\infty}\right)$, we find the following from (61) and (72):

$$
\begin{gathered}
\left\|\bar{M}_{1} \psi\right\|_{L^{2}(Q)^{N}}^{2}+\left\|\bar{M}_{2} \psi\right\|_{L^{2}(Q)^{N}}^{2}+\left\|\bar{M}_{3} \tilde{\psi}\right\|_{L^{2}(Q)^{N}}^{2}+\left\|\bar{M}_{4} \tilde{\psi}\right\|_{L^{2}(Q)^{N}}^{2} \\
+s^{3} \lambda^{4} \iint_{Q}\left(\xi^{3}|\psi|^{2}+\tilde{\xi}^{3}|\tilde{\psi}|^{2}\right) \mathrm{d} x \mathrm{~d} t+s \lambda^{2} \iint_{Q}\left(\xi|\nabla \psi|^{2}+\tilde{\xi}|\nabla \tilde{\psi}|^{2}\right) \mathrm{d} x \mathrm{~d} t \\
+s^{2} \lambda^{2} \iint_{\Sigma} \mathrm{e}^{-2 s \alpha} \xi^{2}|\varphi|^{2} \mathrm{~d} \sigma \mathrm{d} t \leq C\left(\lambda \iint_{Q}\left(\mathrm{e}^{-2 s \alpha}+\mathrm{e}^{-2 s \tilde{\alpha}}\right)|G|^{2} \mathrm{~d} x \mathrm{~d} t\right. \\
+s \lambda \iint_{Q}(\xi|\psi \| \Delta \psi|+\tilde{\xi}|\tilde{\psi}||\Delta \tilde{\psi}|) \mathrm{d} x \mathrm{~d} t \\
+s \lambda^{2} \iint_{\omega^{\prime} \times(0, T)}\left(\xi|\nabla \psi|^{2}+\tilde{\xi}|\nabla \tilde{\psi}|^{2}\right) \mathrm{d} x \mathrm{~d} t \\
\left.+s^{3} \lambda^{4} \iint_{\omega^{\prime} \times(0, T)}\left(\xi^{3}|\psi|^{2}+\tilde{\xi}^{3}|\tilde{\psi}|^{2}\right) \mathrm{d} x \mathrm{~d} t\right),
\end{gathered}
$$

for any $\lambda \geq C \mathrm{e}^{C T\|A\|_{P}^{2}}\left(1+\|A\|_{\infty}+\|A\|_{P}^{5}\right)$ and any $s \geq C \mathrm{e}^{4 \lambda\left\|\eta^{0}\right\|_{\infty}}\left(T^{6}+T^{8}\right)$. Recall that $P \subset L^{\infty}(\Omega)$ continuously, so it suffices to take $\lambda \geq C \mathrm{e}^{C T\|A\|_{P}^{2}}\left(1+\|A\|_{P}^{5}\right)$.

The next step will be to eliminate the local terms of $\nabla \psi$ and $\nabla \tilde{\psi}$ as well as the terms of $|\psi||\Delta \psi|$ and $|\tilde{\psi} \| \Delta \tilde{\psi}|$. To this end, we are going to add integrals of $\Delta \psi$ and $\Delta \tilde{\psi}$ in the left hand side of (74). This will be done taking advantage of the presence of $\bar{M}_{1} \psi$ and $\bar{M}_{3} \tilde{\psi}$. Indeed, from

$$
\Delta \psi=-\bar{M}_{1} \psi-s^{2} \lambda^{2}\left|\nabla \eta^{0}\right|^{2} \xi^{2} \psi-s \alpha_{t} \psi-s \lambda \xi\left(\nabla \nabla \eta^{0} \cdot \psi\right)-s \lambda^{2} \nabla \eta^{0} \xi\left(\psi \nabla \eta^{0}\right)
$$

and

$$
\Delta \tilde{\psi}=-\bar{M}_{3} \tilde{\psi}-s^{2} \lambda^{2}\left|\nabla \eta^{0}\right|^{2} \tilde{\xi}^{2} \tilde{\psi}-s \tilde{\alpha}_{t} \tilde{\psi}+s \lambda \tilde{\xi}\left(\nabla \nabla \eta^{0} \cdot \tilde{\psi}\right)-s \lambda^{2} \nabla \eta^{0} \tilde{\xi}\left(\tilde{\psi} \nabla \eta^{0}\right)
$$

we find

$$
\begin{aligned}
& s^{-1} \iint_{Q}\left(\xi^{-1}|\Delta \psi|^{2}+\tilde{\xi}^{-1}|\Delta \tilde{\psi}|^{2}\right) \mathrm{d} x \mathrm{~d} t \\
& \leq C\left(s^{-1} \iint_{Q}\left(\xi^{-1}\left|\bar{M}_{1} \psi\right|^{2}+\tilde{\xi}^{-1}\left|\bar{M}_{3} \tilde{\psi}\right|^{2}\right) \mathrm{d} x \mathrm{~d} t\right. \\
& +s^{3} \lambda^{4} \iint_{Q}\left(\xi^{3}|\psi|^{2}+\tilde{\xi}^{3}|\tilde{\psi}|^{2}\right) \mathrm{d} x \mathrm{~d} t
\end{aligned}
$$

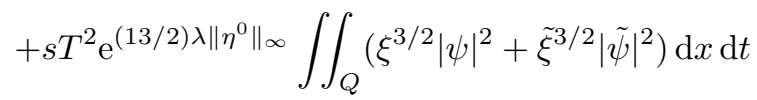

$$
\begin{aligned}
& \left.+s \lambda^{2} \iint_{Q}\left(\xi|\psi|^{2}+\tilde{\xi}|\tilde{\psi}|^{2}\right) \mathrm{d} x \mathrm{~d} t+s \lambda^{4} \iint_{Q}\left(\xi|\psi|^{2}+\tilde{\xi}|\tilde{\psi}|^{2}\right) \mathrm{d} x \mathrm{~d} t\right) .
\end{aligned}
$$


Here, we have employed the bounds of $\alpha_{t}$ and $\tilde{\alpha}_{t}$ given in (55) and (66), respectively. As a consequence, taking again $\lambda \geq C$ and $s \geq C \mathrm{e}^{4 \lambda\left\|\eta^{0}\right\|_{\infty}}\left(T^{7}+T^{8}\right)$, we deduce from (74) that

$$
\begin{aligned}
& \left\|\bar{M}_{2} \psi\right\|_{L^{2}(Q)^{N}}^{2}+\left\|\bar{M}_{4} \tilde{\psi}\right\|_{L^{2}(Q)^{N}}^{2}+s^{3} \lambda^{4} \iint_{Q}\left(\xi^{3}|\psi|^{2}+\tilde{\xi}^{3}|\tilde{\psi}|^{2}\right) \mathrm{d} x \mathrm{~d} t \\
& +s \lambda^{2} \iint_{Q}\left(\xi|\nabla \psi|^{2}+\tilde{\xi}|\nabla \tilde{\psi}|^{2}\right) \mathrm{d} x \mathrm{~d} t \\
& +s^{-1} \iint_{Q}\left(\xi^{-1}|\Delta \psi|^{2}+\tilde{\xi}^{-1}|\Delta \tilde{\psi}|^{2}\right) \mathrm{d} x \mathrm{~d} t+s^{2} \lambda^{2} \iint_{\Sigma} \mathrm{e}^{-2 s \alpha} \xi^{2}|\varphi|^{2} \mathrm{~d} \sigma \mathrm{d} t \\
& \leq C\left(\lambda \iint_{Q}\left(\mathrm{e}^{-2 s \alpha}+\mathrm{e}^{-2 s \tilde{\alpha}}\right)|G|^{2} \mathrm{~d} x \mathrm{~d} t\right. \\
& +s \lambda \iint_{Q}(\xi|\psi||\Delta \psi|+\tilde{\xi}|\tilde{\psi}| \Delta \tilde{\psi} \mid) \mathrm{d} x \mathrm{~d} t \\
& +s \lambda^{2} \iint_{\omega^{\prime} \times(0, T)}\left(\xi|\nabla \psi|^{2}+\tilde{\xi}|\nabla \tilde{\psi}|^{2}\right) \mathrm{d} x \mathrm{~d} t \\
& \left.+s^{3} \lambda^{4} \iint_{\omega^{\prime} \times(0, T)}\left(\xi^{3}|\psi|^{2}+\tilde{\xi}^{3}|\tilde{\psi}|^{2}\right) \mathrm{d} x \mathrm{~d} t\right) .
\end{aligned}
$$

Similar computations lead to an estimate of the term

$$
s^{-1} \iint_{Q}\left(\xi^{-1}\left|\psi_{t}\right|^{2}+\tilde{\xi}^{-1}\left|\tilde{\psi}_{t}\right|^{2}\right) \mathrm{d} x \mathrm{~d} t
$$

in terms of the left hand side of (75), so it can be added there as well.

On the other hand, we have

$s \lambda \iint_{Q}(\xi|\psi||\Delta \psi|+\tilde{\xi}|\tilde{\psi}||\Delta \tilde{\psi}|) \mathrm{d} x \mathrm{~d} t$

$$
\leq C\left(s^{3} \lambda^{3} \iint_{Q}\left(\xi^{3}|\psi|^{2}+\tilde{\xi}^{3}|\tilde{\psi}|^{2}\right) \mathrm{d} x \mathrm{~d} t+s^{-1} \lambda^{-1} \iint_{Q}\left(\xi^{-1}|\Delta \psi|^{2}+\tilde{\xi}^{-1}|\Delta \tilde{\psi}|^{2}\right) \mathrm{d} x \mathrm{~d} t\right) .
$$

This tells that the second term in the right hand side of (75) can be absorbed provided that we take $\lambda \geq C$. Therefore, we obtain

$$
\begin{array}{r}
s^{3} \lambda^{4} \iint_{Q}\left(\xi^{3}|\psi|^{2}+\tilde{\xi}^{3}|\tilde{\psi}|^{2}\right) \mathrm{d} x \mathrm{~d} t+s \lambda^{2} \iint_{Q}\left(\xi|\nabla \psi|^{2}+\tilde{\xi}|\nabla \tilde{\psi}|^{2}\right) \mathrm{d} x \mathrm{~d} t \\
+s^{-1} \iint_{Q}\left(\xi^{-1}\left(\left|\psi_{t}\right|^{2}+|\Delta \psi|^{2}\right)+\tilde{\xi}^{-1}\left(\left|\tilde{\psi}_{t}\right|^{2}+|\Delta \tilde{\psi}|^{2}\right)\right) \mathrm{d} x \mathrm{~d} t \\
+s^{2} \lambda^{2} \iint_{\Sigma} \mathrm{e}^{-2 s \alpha} \xi^{2}|\varphi|^{2} \mathrm{~d} \sigma \mathrm{d} t \leq C\left(\lambda \iint_{Q}\left(\mathrm{e}^{-2 s \alpha}+\mathrm{e}^{-2 s \tilde{\alpha}}\right)|G|^{2} \mathrm{~d} x \mathrm{~d} t\right. \\
+s \lambda^{2} \iint_{\omega^{\prime} \times(0, T)}\left(\xi|\nabla \psi|^{2}+\tilde{\xi}|\nabla \tilde{\psi}|^{2}\right) \mathrm{d} x \mathrm{~d} t \\
\left.+s^{3} \lambda^{4} \iint_{\omega^{\prime} \times(0, T)}\left(\xi^{3}|\psi|^{2}+\tilde{\xi}^{3}|\tilde{\psi}|^{2}\right) \mathrm{d} x \mathrm{~d} t\right)
\end{array}
$$

for any $\lambda \geq C \mathrm{e}^{C T\|A\|_{P}^{2}}\left(1+\|A\|_{P}^{5}\right)$ and any $s \geq C \mathrm{e}^{4 \lambda\left\|\eta^{0}\right\|_{\infty}}\left(T^{6}+T^{8}\right)$. 
Let us now operate with the local term in $\nabla \psi$ (analogous computations can be performed for $\nabla \tilde{\psi}$ ). For this, we introduce a function $\rho \in C^{2}(\Omega)$ such that

$$
\rho \equiv 1 \text { in } \omega^{\prime}, \quad \operatorname{supp} \rho \subset \omega_{0} .
$$

(remind that $\omega_{0}$ was introduced in (49)). Then,

$$
\begin{aligned}
& s \lambda^{2} \iint_{\omega^{\prime} \times(0, T)} \xi|\nabla \psi|^{2} \mathrm{~d} x \mathrm{~d} t \leq \iint_{\omega_{0} \times(0, T)} \rho \xi|\nabla \psi|^{2} \mathrm{~d} x \mathrm{~d} t \\
& =-s \lambda^{2} \iint_{\omega_{0} \times(0, T)} \xi(\nabla \rho \cdot \nabla \psi) \psi \mathrm{d} x \mathrm{~d} t \\
& -s \lambda^{3} \iint_{\omega_{0} \times(0, T)} \rho \xi\left(\nabla \eta^{0} \cdot \nabla \psi\right) \psi \mathrm{d} x \mathrm{~d} t \quad|\nabla \psi|^{2} \mathrm{~d} x \mathrm{~d} t \\
& -s \lambda^{2} \iint_{\omega_{0} \times(0, T)} \rho \xi \Delta \psi \psi \mathrm{d} x \mathrm{~d} t \leq C\left(\lambda^{2} \iint_{\omega_{0} \times(0, T)} \xi^{3}|\psi|^{2} \mathrm{~d} x \mathrm{~d} t\right) \\
& s^{2} \lambda^{4} \iint_{\omega_{0} \times(0, T)} \xi^{2}|\psi|^{2} \mathrm{~d} x \mathrm{~d} t+s^{3} \lambda^{4} \iint_{\omega_{0} \times(0, T)} \xi^{-1}|\Delta \psi|^{2} \mathrm{~d} x \mathrm{~d} t \\
& +\varepsilon s^{-1} \iint_{\omega_{0} \times(0, T)}
\end{aligned}
$$

for a positive constant $\varepsilon=\varepsilon(\Omega, \omega)$ small enough.

Furthermore, from the definition of the weight functions (see (44) above), we find

$$
\mathrm{e}^{-2 s \tilde{\alpha}} \leq \mathrm{e}^{-2 s \alpha}, \quad \tilde{\xi} \leq \xi, \quad|\tilde{\psi}| \leq|\psi| \text { in } Q
$$

Combining all this with (76), we get

$$
\begin{aligned}
& s^{3} \lambda^{4} \iint_{Q} \xi^{3}|\psi|^{2} \mathrm{~d} x \mathrm{~d} t+s \lambda^{2} \iint_{Q} \xi|\nabla \psi|^{2} \mathrm{~d} x \mathrm{~d} t \\
& +s^{-1} \iint_{Q} \xi^{-1}\left(\left|\psi_{t}\right|^{2}+|\Delta \psi|^{2}\right) \mathrm{d} x \mathrm{~d} t+s^{2} \lambda^{2} \iint_{\Sigma} \mathrm{e}^{-2 s \alpha} \xi^{2}|\varphi|^{2} \mathrm{~d} \sigma \mathrm{d} t \\
& \leq C\left(\lambda \iint_{Q} \mathrm{e}^{-2 s \alpha}|G|^{2} \mathrm{~d} x \mathrm{~d} t+s^{3} \lambda^{4} \iint_{\omega_{0} \times(0, T)} \xi^{3}|\psi|^{2} \mathrm{~d} x \mathrm{~d} t\right),
\end{aligned}
$$

for any $\lambda \geq C \mathrm{e}^{C T\|A\|_{P}^{2}}\left(1+\|A\|_{P}^{5}\right)$ and any $s \geq C \mathrm{e}^{4 \lambda\left\|\eta^{0}\right\|_{\infty}}\left(T^{6}+T^{8}\right)$.

Finally, let us go back to the original variable $\varphi$. For the moment, we have

$$
\begin{aligned}
& s^{3} \lambda^{4} \iint_{Q} \mathrm{e}^{-2 s \alpha} \xi^{3}|\varphi|^{2} \mathrm{~d} x \mathrm{~d} t+s \lambda^{2} \iint_{Q} \xi|\nabla \psi|^{2} \mathrm{~d} x \mathrm{~d} t \\
& +s^{-1} \iint_{Q} \xi^{-1}\left(\left|\psi_{t}\right|^{2}+|\Delta \psi|^{2}\right) \mathrm{d} x \mathrm{~d} t+s^{2} \lambda^{2} \iint_{\Sigma} \mathrm{e}^{-2 s \alpha} \xi^{2}|\varphi|^{2} \mathrm{~d} \sigma \mathrm{d} t \\
& \leq C\left(\lambda \iint_{Q} \mathrm{e}^{-2 s \alpha}|G|^{2} \mathrm{~d} x \mathrm{~d} t+s^{3} \lambda^{4} \iint_{\omega_{0} \times(0, T)} \mathrm{e}^{-2 s \alpha} \xi^{3}|\varphi|^{2} \mathrm{~d} x \mathrm{~d} t\right),
\end{aligned}
$$




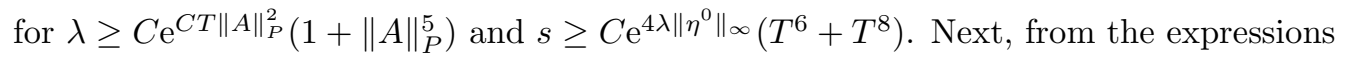

and

$$
\begin{gathered}
\nabla \varphi=\mathrm{e}^{s \alpha}\left(-s \lambda \nabla \eta^{0} \xi \psi+\nabla \psi\right), \\
\Delta \varphi=\mathrm{e}^{s \alpha}\left(s^{2} \lambda^{2}\left|\nabla \eta^{0}\right|^{2} \xi^{2} \psi-2 s \lambda \xi\left(\nabla \eta^{0} \nabla \psi\right)+\Delta \psi-s \lambda \Delta \eta^{0} \xi \psi-s \lambda^{2}\left|\nabla \eta^{0}\right|^{2} \xi \psi\right),
\end{gathered}
$$

we find the following estimates:

$$
\varphi_{t}=\mathrm{e}^{s \alpha}\left(s \alpha_{t} \psi+\psi_{t}\right)
$$

$$
\begin{aligned}
& s \lambda^{2} \iint_{Q} \mathrm{e}^{-2 s \alpha} \xi|\nabla \varphi|^{2} \mathrm{~d} x \mathrm{~d} t \leq C\left(s^{3} \lambda^{4} \iint_{Q} \xi^{3}|\psi|^{2} \mathrm{~d} x \mathrm{~d} t+s \lambda^{2} \iint_{Q} \xi|\nabla \psi|^{2} \mathrm{~d} x \mathrm{~d} t\right), \\
& s^{-1} \iint_{Q} \mathrm{e}^{-2 s \alpha} \xi^{-1}|\Delta \varphi|^{2} \mathrm{~d} x \mathrm{~d} t \leq C\left(s^{3} \lambda^{4} \iint_{Q} \xi^{3}|\psi|^{2} \mathrm{~d} x \mathrm{~d} t+s \lambda^{2} \iint_{Q} \xi|\nabla \psi|^{2} \mathrm{~d} x \mathrm{~d} t\right. \\
& \left.\quad+s^{-1} \iint_{Q} \xi^{-1}|\Delta \psi|^{2} \mathrm{~d} x \mathrm{~d} t++s \lambda^{2} \iint_{Q} \xi|\psi|^{2} \mathrm{~d} x \mathrm{~d} t+s \lambda^{4} \iint_{Q} \xi|\psi|^{2} \mathrm{~d} x \mathrm{~d} t\right)
\end{aligned}
$$

and

$$
s^{-1} \iint_{Q} \mathrm{e}^{-2 s \alpha} \xi^{-1}\left|\varphi_{t}\right|^{2} \mathrm{~d} x \mathrm{~d} t \leq C\left(s T^{2} \mathrm{e}^{4 \lambda\left\|\eta^{0}\right\|} \iint_{Q} \xi^{3 / 2}|\psi|^{2} \mathrm{~d} x \mathrm{~d} t+s^{-1} \iint_{Q} \xi^{-1}\left|\psi_{t}\right|^{2} \mathrm{~d} x \mathrm{~d} t\right)
$$

Now, it is not difficult to see that the bounds of these three terms can be estimated by the left hand side of (78) if we take $\lambda \geq C$ and $s \geq C \mathrm{e}^{2 \lambda\left\|\eta^{0}\right\|_{\infty}}\left(T^{7}+T^{8}\right)$. As a conclusion, these considerations and the inequality (79) provide the desired estimate (47), namely:

$$
\begin{aligned}
& s^{3} \lambda^{4} \iint_{Q} \mathrm{e}^{-2 s \alpha} \xi^{3}|\varphi|^{2} \mathrm{~d} x \mathrm{~d} t+s \lambda^{2} \iint_{Q} \xi|\nabla \psi|^{2} \mathrm{~d} x \mathrm{~d} t \\
& +s^{-1} \iint_{Q} \xi^{-1}\left(\left|\psi_{t}\right|^{2}+|\Delta \psi|^{2}\right) \mathrm{d} x \mathrm{~d} t+s^{2} \lambda^{2} \iint_{\Sigma} \mathrm{e}^{-2 s \alpha} \xi^{2}|\varphi|^{2} \mathrm{~d} \sigma \mathrm{d} t \\
& \leq C\left(\lambda \iint_{Q} \mathrm{e}^{-2 s \alpha}|G|^{2} \mathrm{~d} x \mathrm{~d} t+s^{3} \lambda^{4} \iint_{\omega_{0} \times(0, T)} \mathrm{e}^{-2 s \alpha} \xi^{3}|\varphi|^{2} \mathrm{~d} x \mathrm{~d} t\right),
\end{aligned}
$$

for $\lambda \geq C \mathrm{e}^{C T\|A\|_{P}^{2}}\left(1+\|A\|_{P}^{5}\right)$ and $s \geq C(\Omega, \omega) \mathrm{e}^{4 \lambda\left\|\eta^{0}\right\|_{\infty}}\left(T^{6}+T^{8}\right)$.

Remark 2.2. The computations made in the last step of the proof of Proposition 2.1 prove that, under the "natural" assumption on $A$ to be an uniformly elliptic matrix function, a positive boundary integral comes out when combining all the boundary terms appearing in (61) and (72).

\subsection{Carleman inequality for the Stokes system}

In this subsection and as a consequence of inequality (47), we will deduce a Carleman kind estimate for the system (11). This will serve us to prove the null controllability of system (2) in the next section.

Proposition 2.3. Let us suppose that $a$ and b satisfy hypothesis (7) and A satisfy (8)-(10). Then, there exist three positive constants $\tilde{\lambda}, \tilde{s}$ and $\tilde{C}$ depending on $\Omega$ and $\omega$ such that,

$$
I(s, \lambda ; \varphi) \leq C(1+T) s^{15 / 2} \lambda^{8} \iint_{\omega \times(0, T)} \mathrm{e}^{-4 s \widehat{\alpha}+2 s \alpha^{*}} \widehat{\xi}^{15 / 2}|\varphi|^{2} \mathrm{~d} x \mathrm{~d} t
$$


for any $\left.\lambda \geq \tilde{\lambda} \mathrm{e}^{\tilde{\lambda} T\left(\|a\|_{\infty}^{2}+\|b\|_{\infty}^{2}+\|A\|_{P}^{2}\right.}\right)\left(1+\|a\|_{\infty}^{10 / 3}+\|b\|_{\infty}^{10 / 3}+\left\|a_{t}\right\|_{L^{2}\left(L^{r}\right)}^{10 / 3}+\left\|b_{t}\right\|_{L^{2}\left(L^{r}\right)}^{10 / 3}+\|A\|_{P}^{5}+\|A\|_{H^{(3-\ell) / 2}\left(H^{\nu_{2}}\right)}^{10 / 3}\right)$, any $s \geq \tilde{s} \mathrm{e}^{8 \lambda\left\|\eta^{0}\right\|_{\infty}}\left(T^{4}+T^{8}\right)$ and any $\varphi^{0} \in H$, where $\varphi$ is the corresponding solution to (11). Here, we remind that the definition of $I(s, \lambda ; \varphi)$ was given in (48).

Proof. Let us remark that this proof follows the same ideas of the Carleman inequalities for Stokes systems with Dirichlet conditions, which have been proved in $[8,13]$.

We start applying inequality (47) with a right hand side

$$
G=-\nabla \pi+(a, \nabla) \varphi+D \varphi b
$$

This yields

$$
\begin{gathered}
I(s, \lambda ; \varphi) \leq\left(s^{3} \lambda^{4} \iint_{\omega_{0} \times(0, T)} \mathrm{e}^{-2 s \alpha} \xi^{3}|\varphi|^{2} \mathrm{~d} x \mathrm{~d} t+\lambda \iint_{Q} \mathrm{e}^{-2 s \alpha}|\nabla \pi|^{2} \mathrm{~d} x \mathrm{~d} t\right. \\
\left.+\lambda\left(\|a\|_{\infty}^{2}+\|b\|_{\infty}^{2}\right) \iint_{Q} \mathrm{e}^{-2 s \alpha}|\nabla \varphi|^{2} \mathrm{~d} x \mathrm{~d} t\right),
\end{gathered}
$$

for $\lambda \geq C \mathrm{e}^{C T\|A\|_{P}^{2}}\left(1+\|A\|_{P}^{5}\right)$ and $s \geq \bar{s} \mathrm{e}^{4 \lambda\left\|\eta^{0}\right\|_{\infty}}\left(T^{6}+T^{8}\right)$.

Now, we take $\lambda \geq C\left(\|a\|_{\infty}^{2}+\|b\|_{\infty}^{2}\right)$, so that the last term can be eliminated using the term in $s \lambda^{2}$ of $I(s, \lambda ; \varphi)$. Consequently, we have

$$
I(s, \lambda ; \varphi) \leq C\left(s^{3} \lambda^{4} \iint_{\omega_{0} \times(0, T)} \mathrm{e}^{-2 s \alpha} \xi^{3}|\varphi|^{2} \mathrm{~d} x \mathrm{~d} t+\lambda \iint_{Q} \mathrm{e}^{-2 s \alpha}|\nabla \pi|^{2} \mathrm{~d} x \mathrm{~d} t\right)
$$

for any $\lambda \geq C \mathrm{e}^{C T\|A\|_{P}^{2}}\left(1+\|a\|_{\infty}^{2}+\|b\|_{\infty}^{2}+\|A\|_{P}^{5}\right)$ and any $s \geq C \mathrm{e}^{4 \lambda\left\|\eta^{0}\right\|_{\infty}}\left(T^{6}+T^{8}\right)$.

The next step will be to localize the pressure term. This will be made by means of an elliptic Carleman inequality which has been proved in [14]. Indeed, let us take the divergence operator in the equation verified by $\varphi$. Then,

$$
\Delta \pi(t)=\nabla \cdot((a, \nabla) \varphi+D \varphi b)(t) \text { in } \Omega \text { a.e. } t \in(0, T)
$$

We see the expression in the right hand side as a $H^{-1}$ term and we apply that result. Thus, there exist two constants $\tilde{\tau}$ and $\tilde{\lambda}$ greater than 1 , such that

$$
\begin{aligned}
\int_{\Omega} \mathrm{e}^{2 \tau \eta}|\nabla \pi(t)|^{2} \mathrm{~d} x+ & \tau^{2} \lambda^{2} \int_{\Omega} \mathrm{e}^{2 \tau \eta} \eta^{2}|\pi(t)|^{2} \mathrm{~d} x \\
& \leq C\left(\tau\left(\|a\|_{\infty}^{2}+\|b\|_{\infty}^{2}\right) \int_{\Omega} \mathrm{e}^{2 \tau \eta} \eta|\nabla \varphi(t)|^{2} \mathrm{~d} x+\tau^{1 / 2} \mathrm{e}^{2 \tau}\|\pi(t)\|_{H^{1 / 2}(\partial \Omega)}^{2}\right. \\
& \left.+\tau^{2} \lambda^{2} \int_{\omega^{\prime}} \mathrm{e}^{2 \tau \eta} \eta^{2}|\pi(t)|^{2} \mathrm{~d} x+\int_{\omega^{\prime}} \mathrm{e}^{2 \tau \eta}|\nabla \pi(t)|^{2} \mathrm{~d} x\right)
\end{aligned}
$$

for $\tau \geq \tilde{\tau}$ and $\lambda \geq \tilde{\lambda}$. Here, the function $\eta$ is given by

$$
\eta(x)=\mathrm{e}^{\lambda \eta^{0}(x)} \quad \text { in } \Omega
$$

for each $\lambda>0$. We remind that the function $\eta^{0}$ was introduced in (45).

Let us now set

$$
\tau=\frac{s}{t^{4}(T-t)^{4}}
$$


and multiply the previous inequality by

$$
\exp \left\{-2 s \frac{\mathrm{e}^{2 \lambda\left\|\eta^{0}\right\|_{\infty}}}{t^{4}(T-t)^{4}}\right\}
$$

Then, an integration in time gives

$$
\begin{aligned}
\iint_{Q} \mathrm{e}^{-2 s \alpha}|\nabla \pi|^{2} \mathrm{~d} x \mathrm{~d} t+s^{2} \lambda^{2} \iint_{Q} \mathrm{e}^{-2 s \alpha} \xi^{2}|\pi|^{2} \mathrm{~d} x \mathrm{~d} t \\
\leq C\left(s\left(\|a\|_{\infty}^{2}+\|b\|_{\infty}^{2}\right) \iint_{Q} \mathrm{e}^{-2 s \alpha} \xi|\nabla \varphi|^{2} \mathrm{~d} x \mathrm{~d} t\right. \\
\quad+s^{1 / 2} \int_{0}^{T} \mathrm{e}^{-2 s \alpha^{*}}\left(\xi^{*}\right)^{1 / 2}\|\pi(t)\|_{H^{1 / 2}(\partial \Omega)}^{2} \\
\quad+s^{2} \lambda^{2} \iint_{\omega^{\prime} \times(0, T)} \mathrm{e}^{-2 s \alpha} \xi^{2}|\pi|^{2} \mathrm{~d} x \mathrm{~d} t \\
\left.\quad+\iint_{\omega^{\prime} \times(0, T)} \mathrm{e}^{-2 s \alpha}|\nabla \pi|^{2} \mathrm{~d} x \mathrm{~d} t\right)
\end{aligned}
$$

for $\lambda \geq C$ and $s \geq C T^{8}$. We remind that the definitions of $\alpha^{*}$ and $\xi^{*}$ were given in (44). Observe that taking $\lambda \geq C\left(\|a\|_{\infty}^{2}+\|b\|_{\infty}^{2}\right)$ and $s \geq C T^{8}$, the first term in the right will be absorbed when combined with (81). On the other hand, let us introduce the following functions, in order to estimate the term of the trace:

$$
\widetilde{\varphi}=s^{1 / 4} \mathrm{e}^{-s \alpha^{*}}\left(\xi^{*}\right)^{1 / 4} \varphi, \quad \widetilde{\pi}=s^{1 / 4} \mathrm{e}^{-s \alpha^{*}}\left(\xi^{*}\right)^{1 / 4} \pi
$$

They verify

$$
\left\{\begin{array}{lc}
-\widetilde{\varphi}_{t}-\Delta \widetilde{\varphi}-(a(x, t), \nabla) \widetilde{\varphi}-D \widetilde{\varphi} b(x, t)+\nabla \widetilde{\pi} & =-\left(s^{1 / 4} \mathrm{e}^{-s \alpha^{*}}\left(\xi^{*}\right)^{1 / 4}\right)_{t}(t) \varphi \\
& \text { in } Q, \\
\nabla \cdot \widetilde{\varphi}=0 & \text { in } Q, \\
\widetilde{\varphi} \cdot n=0, \quad(\sigma(\widetilde{\varphi}, \widetilde{\pi}))_{t g}+(A(x, t) \widetilde{\varphi})_{t g}=0 & \text { on } \Sigma, \\
\widetilde{\varphi}(\cdot, T)=0 & \text { in } \Omega .
\end{array}\right.
$$

Let us first watch $\widetilde{\varphi}$ as the weak solution to (84). In particular, it satisfies

$$
\|\widetilde{\varphi}\|_{L^{2}\left(H^{1}\right)}^{2} \leq \mathrm{e}^{C T\left(\|a\|_{\infty}^{2}+\|b\|_{\infty}^{2}+\|A\|_{\infty}^{2}\right)}\left\|s^{1 / 4}\left(\mathrm{e}^{-s \alpha^{*}}\left(\xi^{*}\right)^{1 / 4}\right)_{t} \varphi\right\|_{L^{2}(Q)^{N}}^{2}
$$

Applying now Proposition 1.1 and taking into account that $P \subset L^{\infty}(\Omega)$ continuously, we find

$$
\|\widetilde{\pi}\|_{L^{2}\left(H^{1}\right)}^{2} \leq C \mathrm{e}^{C T\left(\|a\|_{\infty}^{2}+\|b\|_{\infty}^{2}+\|A\|_{P}^{2}\right)}\left(1+\|A\|_{P}^{4}\right)
$$

$$
\times\left(1+\|a\|_{\infty}^{2}+\|b\|_{\infty}^{2}\right) s^{5 / 2} T^{2} \mathrm{e}^{4 \lambda\left\|\eta^{0}\right\|_{\infty}} \iint_{Q} \mathrm{e}^{-2 s \alpha^{*}}\left(\xi^{*}\right)^{3}|\varphi|^{2} \mathrm{~d} x \mathrm{~d} t
$$


Plugging this into (83) and taking $\lambda \geq C \mathrm{e}^{C T\left(\|a\|_{\infty}^{2}+\|b\|_{\infty}^{2}+\|A\|_{P}^{2}\right)}\left(1+\|a\|_{\infty}^{2}+\|b\|_{\infty}^{2}+\|A\|_{P}^{5}\right)$ and $s \geq C \mathrm{e}^{8 \lambda\left\|\eta^{0}\right\|{ }_{\infty} T^{4}}$, we get

$$
\begin{aligned}
& \iint_{Q} \mathrm{e}^{-2 s \alpha}|\nabla \pi|^{2} \mathrm{~d} x \mathrm{~d} t+s^{2} \lambda^{2} \iint_{Q} \mathrm{e}^{-2 s \alpha} \xi^{2}|\pi|^{2} \mathrm{~d} x \mathrm{~d} t \leq C\left(s\left(\|a\|_{\infty}^{2}+\|b\|_{\infty}^{2}\right) \iint_{Q} \mathrm{e}^{-2 s \alpha} \xi|\nabla \varphi|^{2} \mathrm{~d} x \mathrm{~d} t\right. \\
& \left.+s^{2} \lambda^{2} \iint_{\omega^{\prime} \times(0, T)} \mathrm{e}^{-2 s \alpha} \xi^{2}|\pi|^{2} \mathrm{~d} x \mathrm{~d} t+\iint_{\omega^{\prime} \times(0, T)} \mathrm{e}^{-2 s \alpha}|\nabla \pi|^{2} \mathrm{~d} x \mathrm{~d} t\right)+\varepsilon s^{3} \lambda^{4} \iint_{Q} \mathrm{e}^{-2 s \alpha} \xi^{3}|\varphi|^{2} \mathrm{~d} x \mathrm{~d} t
\end{aligned}
$$

for a small positive constant $\varepsilon(\Omega, \omega)$.

Combining this with (81), we have

$$
\begin{aligned}
& I(s, \lambda ; \varphi) \leq C\left(s^{3} \lambda^{4} \iint_{\omega_{0} \times(0, T)} \mathrm{e}^{-2 s \alpha} \xi^{3}|\varphi|^{2} \mathrm{~d} x \mathrm{~d} t\right. \\
& \left.+s^{2} \lambda^{3} \iint_{\omega^{\prime} \times(0, T)} \mathrm{e}^{-2 s \alpha} \xi^{2}|\pi|^{2} \mathrm{~d} x \mathrm{~d} t+\lambda \iint_{\omega^{\prime} \times(0, T)} \mathrm{e}^{-2 s \alpha}|\nabla \pi|^{2} \mathrm{~d} x \mathrm{~d} t\right)
\end{aligned}
$$

for $\lambda \geq C \mathrm{e}^{C T\left(\|a\|_{\infty}^{2}+\|b\|_{\infty}^{2}+\|A\|_{P}^{2}\right)}\left(1+\|a\|_{\infty}^{2}+\|b\|_{\infty}^{2}+\|A\|_{P}^{5}\right)$ and $s \geq C \mathrm{e}^{8 \lambda\left\|\eta^{0}\right\|_{\infty}}\left(T^{4}+T^{8}\right)$.

The last task will be to bound the local terms involving the pressure. To do this, we will follow the same ideas developed in [8], so we may sometimes give few details along the proof. Indeed, let us take the pressure $\pi$ to satisfy

$$
\int_{\omega^{\prime}} \pi(t) \mathrm{d} x=0 \text { a.e. } t \in(0, T) .
$$

Then, by virtue of Poincare-Wirtinger's inequality and the definition of $\widehat{\alpha}$ and $\widehat{\xi}$ (see (44)), we find

$$
\begin{gathered}
s^{2} \lambda^{3} \iint_{\omega^{\prime} \times(0, T)} \mathrm{e}^{-2 s \alpha} \xi^{2}|\pi|^{2} \mathrm{~d} x \mathrm{~d} t \leq s^{2} \lambda^{3} \iint_{\omega^{\prime} \times(0, T)} \mathrm{e}^{-2 s \widehat{\alpha} \widehat{\xi}^{2}|\pi|^{2} \mathrm{~d} x \mathrm{~d} t} \\
\leq C s^{2} \lambda^{3} \iint_{\omega^{\prime} \times(0, T)} \mathrm{e}^{-2 s \widehat{\alpha} \widehat{\xi}^{2}|\nabla \pi|^{2} \mathrm{~d} x \mathrm{~d} t,}
\end{gathered}
$$

for some positive constant $C(\Omega, \omega)$.

Moreover, from the differential equation in (11), we have

$$
\nabla \pi=\varphi_{t}+\Delta \varphi+(a(x, t), \nabla) \varphi+D \varphi b(x, t) \text { in } Q,
$$

which connected with (86), gives

$$
\begin{aligned}
& I(s, \lambda ; \varphi) \leq C\left(s^{3} \lambda^{4} \iint_{\omega_{0} \times(0, T)} \mathrm{e}^{-2 s \alpha} \xi^{3}|\varphi|^{2} \mathrm{~d} x \mathrm{~d} t\right. \\
& \quad+s^{2} \lambda^{3} \iint_{\omega^{\prime} \times(0, T)} \mathrm{e}^{-2 s \widehat{\alpha} \widehat{\xi}^{2}\left|\varphi_{t}\right|^{2} \mathrm{~d} x \mathrm{~d} t} \\
& \quad+s^{2} \lambda^{3} \iint_{\omega^{\prime} \times(0, T)} \mathrm{e}^{-2 s \widehat{\alpha} \widehat{\xi}^{2}|\Delta \varphi|^{2} \mathrm{~d} x \mathrm{~d} t} \\
& \quad+s^{2} \lambda^{3}\left(\|a\|_{\infty}^{2}+\|b\|_{\infty}^{2}\right) \iint_{\omega^{\prime} \times(0, T)} \mathrm{e}^{\left.-2 s \widehat{\alpha} \widehat{\xi}^{2}|\nabla \varphi|^{2} \mathrm{~d} x \mathrm{~d} t\right),}
\end{aligned}
$$

for $\lambda \geq C \mathrm{e}^{C T\left(\|a\|_{\infty}^{2}+\|b\|_{\infty}^{2}+\|A\|_{P}^{2}\right)}\left(1+\|a\|_{\infty}^{2}+\|b\|_{\infty}^{2}+\|A\|_{P}^{5}\right)$ and $s \geq C \mathrm{e}^{8 \lambda\left\|\eta^{0}\right\| \|_{\infty}}\left(T^{4}+T^{8}\right)$. 
Let us denote

$$
\widehat{\theta}(t)=s \lambda^{3 / 2} \mathrm{e}^{-\widehat{\alpha}(t)} \widehat{\xi}(t)
$$

At this point, we use a local estimate of $\Delta \varphi$ proved in [8] (see step 4 in the proof of theorem 1), namely:

$$
\begin{gathered}
\iint_{\omega^{\prime} \times(0, T)}|\widehat{\theta}|^{2}|\Delta \varphi|^{2} \mathrm{~d} x \mathrm{~d} t \leq C(1+T)\left(\iint_{\omega_{0} \times(0, T)}\left|\widehat{\theta}^{\prime}\right|^{2}|\varphi|^{2} \mathrm{~d} x \mathrm{~d} t\right. \\
\left.\quad+\iint_{\omega_{0} \times(0, T)}|\widehat{\mid}|^{2}\left(|(a, \nabla) \varphi|^{2}+|D \varphi b|^{2}+|\varphi|^{2}\right) \mathrm{d} x \mathrm{~d} t\right) .
\end{gathered}
$$

Let us combine this inequality with (87). We obtain

$$
\begin{aligned}
& I(s, \lambda ; \varphi) \leq C(1+T)\left(s^{4} \lambda^{4} T^{2} \mathrm{e}^{4 \lambda\left\|\eta^{0}\right\|_{\infty}} \iint_{\omega^{\prime} \times(0, T)} \mathrm{e}^{-2 s \widehat{\alpha} \widehat{\xi}^{9 / 2}|\varphi|^{2} \mathrm{~d} x \mathrm{~d} t}\right. \\
& \quad+s^{2} \lambda^{3} \iint_{\omega^{\prime} \times(0, T)} \mathrm{e}^{-2 s \widehat{\alpha} \widehat{\xi}^{2}\left|\varphi_{t}\right|^{2} \mathrm{~d} x \mathrm{~d} t} \\
& \quad+s^{2} \lambda^{3}\left(\|a\|_{\infty}^{2}+\|b\|_{\infty}^{2}\right) \iint_{\omega_{1} \times(0, T)} \mathrm{e}^{\left.-2 s \widehat{\alpha} \widehat{\xi}^{2}|\nabla \varphi|^{2} \mathrm{~d} x \mathrm{~d} t\right),}
\end{aligned}
$$

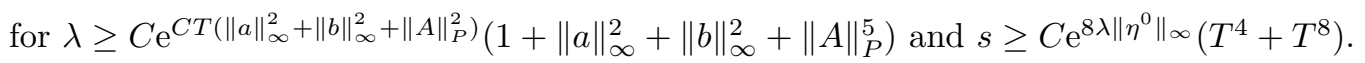

It only rests to estimate $\varphi_{t}$. For this, we integrate by parts with respect to $t$ :

$$
\begin{aligned}
s^{2} \lambda^{3} \iint_{\omega^{\prime} \times(0, T)} \mathrm{e}^{-2 s \widehat{\alpha}} \widehat{\xi}^{2}\left|\varphi_{t}\right|^{2} \mathrm{~d} x \mathrm{~d} t=\frac{1}{2} s^{2} \lambda^{3} \iint_{\omega^{\prime} \times(0, T)}\left(\mathrm{e}^{-2 s \widehat{\alpha}} \widehat{\xi}^{2}\right) t t|\varphi|^{2} \mathrm{~d} x \mathrm{~d} t & -s^{2} \lambda^{3} \iint_{\omega^{\prime} \times(0, T)} \mathrm{e}^{-2 s \widehat{\alpha}} \widehat{\xi}^{2} \varphi \cdot \varphi_{t t} \mathrm{~d} x \mathrm{~d} t
\end{aligned}
$$

It is not difficult to see that $\left(\mathrm{e}^{-2 s \widehat{\alpha}} \widehat{\xi}^{2}\right)_{t t}$ is a function bounded by

$$
C s^{2} T^{2} \mathrm{e}^{-2 s \widehat{\alpha}} \widehat{\xi}^{9 / 2}
$$

On the other hand, let us apply Hölder's inequality in the last term:

$$
\begin{aligned}
& s^{2} \lambda^{3} \iint_{\omega^{\prime} \times(0, T)} \mathrm{e}^{-2 s \widehat{\alpha} \widehat{\xi}^{2}} \varphi \cdot \varphi_{t t} \mathrm{~d} x \mathrm{~d} t \\
& \leq\left(s^{15 / 2} \lambda^{8} \iint_{\omega^{\prime} \times(0, T)} \mathrm{e}^{-4 s \widehat{\alpha}+2 s \alpha^{*}} \widehat{\xi}^{-15 / 2}|\varphi|^{2} \mathrm{~d} x \mathrm{~d} t+\iint_{\omega^{\prime} \times(0, T)}\left|\theta^{*}\right|^{2}\left|\varphi_{t t}\right|^{2} \mathrm{~d} x \mathrm{~d} t\right)
\end{aligned}
$$

where we have denoted

$$
\theta^{*}=s^{-7 / 4} \lambda^{-1} \mathrm{e}^{-s \alpha^{*}} \widehat{\xi}^{-7 / 4}
$$

Let us now introduce the functions $u=\theta^{*} \varphi_{t}$ and $h=\theta^{*} \pi_{t}$. They verify

$$
\begin{cases}-u_{t}-\Delta u-(a(x, t), \nabla) u-D u b(x, t)+\nabla h=G & \text { in } Q \\ \nabla \cdot u=0 & \text { in } Q \\ u \cdot n=0,(\sigma(u, h) \cdot n)_{t g}+(A(x, t) u)_{t g}=-\theta^{*} A_{t}(x, t) \varphi & \text { on } \Sigma \\ u(\cdot, T)=0 & \text { in } \Omega\end{cases}
$$


with

$$
G=-\theta_{t}^{*} \varphi_{t}+\theta^{*}\left(a_{t}, \nabla\right) \varphi+\theta^{*} D \varphi b_{t}
$$

The idea we develop now was already presented in Proposition 1.1, but in a different way. On the other hand, we will keep an explicit dependence on the coefficients $a, b$ and $A$.

We first define $u$ as the weak solution of (91). In view of (20), it suffices to have $G \in L^{2}\left(0, T ; H^{-1}(\Omega)^{N}\right)$ and $\theta^{*} A_{t} \varphi \in L^{2}\left(0, T ; H^{-1 / 2}(\partial \Omega)^{N}\right)$. We already have that $\varphi$ is a strong solution, i.e.,

$$
\varphi \in L^{2}\left(0, T ; H^{2}(\Omega)^{N}\right) \cap H^{1}\left(0, T ; L^{2}(\Omega)^{N}\right) .
$$

Then, the hypothesis (7) on $a$ and $b$ and (10) (on $A$ ) readily imply that $G \in L^{2}\left(0, T ; H^{-1}(\Omega)^{N}\right)$ and that $\theta^{*} A_{t} \varphi \in L^{2}\left(0, T ; H^{-1 / 2}(\partial \Omega)^{N}\right)$. Moreover,

$$
\|u\|_{L^{2}\left(H^{1}\right)}^{2} \leq C \mathrm{e}^{C T\left(\|a\|_{\infty}^{2}+\|b\|_{\infty}^{2}+\|A\|_{\infty}^{2}\right)}\left(\|G\|_{L^{2}\left(H^{-1}\right)}^{2}+\left\|\theta^{*} A_{t} \varphi\right\|_{L^{2}\left(H^{-1 / 2}\right)}^{2}\right) .
$$

Next, we see $u$ as the strong solution of (91). By virtue of Proposition 1.1, we must verify that $G \in L^{2}(Q)^{N}$ and $\theta^{*} A_{t} \varphi \in H^{(1-\ell) / 2}\left(0, T ; H^{\ell-1 / 2}(\partial \Omega)^{N}\right) \cap L^{2}\left(0, T ; H^{1 / 2}(\partial \Omega)^{N}\right)$.

Let us first show that $G \in L^{2}(Q)^{N}$. The only terms to study are $\theta^{*}\left(a_{t}, \nabla\right) \varphi$ and $\theta^{*} D \varphi b_{t}$. Since $u \in$ $L^{2}\left(0, T ; H^{1}(\Omega)^{N}\right)$, we have $\theta^{*} \nabla \varphi \in H^{1}\left(0, T ; L^{2}(\Omega)^{N \times N}\right)$. This, together with $\theta^{*} \nabla \varphi \in L^{2}\left(0, T ; H^{1}(\Omega)^{N \times N}\right)$, gives (see [21] for more details)

$$
\theta^{*} \nabla \varphi \in L^{\infty}\left(0, T ; H^{1 / 2}(\Omega)^{N \times N}\right) .
$$

Then, (7) readily yields $\theta^{*}\left(a_{t}, \nabla\right) \varphi \in L^{2}(Q)^{N}, \theta^{*} D \varphi b_{t} \in L^{2}(Q)^{N}$ and

$$
\left\|\theta^{*}\left(a_{t}, \nabla\right) \varphi\right\|_{L^{2}(Q)^{N}}^{2}+\left\|\theta^{*} D \varphi b_{t}\right\|_{L^{2}(Q)^{N}}^{2} \leq C\left(\left\|a_{t}\right\|_{L^{2}\left(L^{r}\right)}^{2}+\left\|b_{t}\right\|_{L^{2}\left(L^{r}\right)}\right)\left(\left\|\theta^{*} \varphi\right\|_{L^{2}\left(H^{2}\right)}^{2}+\left\|\theta_{t}^{*} \varphi\right\|_{L^{2}(W)}^{2}+\|u\|_{L^{2}(W)}^{2}\right) .
$$

Moreover, from (92) we have $\theta^{*} \varphi \in H^{1}\left(0, T ; H^{1 / 2}(\partial \Omega)^{N}\right)$ which combined with (10) gives

$$
\theta^{*} A_{t} \varphi \in H^{(1-\ell) / 2}\left(0, T ; H^{\nu_{2}}(\partial \Omega)^{N}\right) \subset H^{(1-\ell) / 2}\left(0, T ; H^{\ell-1 / 2}(\partial \Omega)^{N}\right)
$$

$\left(\nu_{2}\right.$ was defined in the introduction) and

$$
\left\|\theta^{*} A_{t} \varphi\right\|_{H^{(1-\ell) / 2}\left(H^{\ell-1 / 2}\right)}^{2} \leq C\|A\|_{H^{(3-\ell) / 2}\left(H^{\nu_{2}}\right)}^{2}\left(\left\|\theta_{t}^{*} \varphi\right\|_{L^{2}\left(H^{1}\right)}^{2}+\|u\|_{L^{2}\left(H^{1}\right)}^{2}\right) .
$$

Additionally, from $\theta^{*} \varphi \in L^{2}\left(0, T ; H^{2}(\Omega)^{N}\right) \cap H^{1}\left(0, T ; H^{1}(\Omega)^{N}\right)$ we find that $\theta^{*} \varphi$ belongs to $H^{1 / 4}\left(0, T ; H^{5 / 4}(\partial \Omega)^{N}\right)$. Thus, using $A_{t} \in H^{(1-\ell) / 2}\left(0, T ; H^{1 / 2}(\partial \Omega)^{N \times N}\right)$ (deduced from $(10)$ ), we have $\theta^{*} A_{t} \varphi \in L^{2}\left(0, T ; H^{1 / 2}(\partial \Omega)^{N}\right)$ and

$$
\left\|\theta^{*} A_{t} \varphi\right\|_{L^{2}\left(H^{1 / 2}\right)}^{2} \leq C\|A\|_{H^{(3-\ell) / 2}\left(H^{1 / 2}\right)}^{2}\left(\left\|\theta^{*} \varphi\right\|_{L^{2}\left(H^{2}\right)}^{2}+\left\|\theta_{t}^{*} \varphi\right\|_{L^{2}\left(H^{1}\right)}^{2}+\|u\|_{L^{2}\left(H^{1}\right)}^{2}\right) .
$$

As a conclusion, we deduce that (in particular)

$$
u \in H^{1}\left(0, T ; L^{2}(\Omega)^{N}\right)
$$

and

$$
\begin{aligned}
& \|u\|_{H^{1}\left(L^{2}\right)}^{2} \leq C \mathrm{e}^{C T\|A\|_{P}^{2}}\left(1+\|A\|_{P}^{4}\right)\left(1+\|A\|_{H^{(3-\ell) / 2}\left(H^{\nu_{2}}\right)}^{2}\right) \\
& \times\left(\|G\|_{L^{2}(Q)^{N}}^{2}+\|(a, \nabla) u\|_{L^{2}(Q)^{N}}^{2}+\|D u b\|_{L^{2}(Q)^{N}}^{2}+\left\|\theta^{*} A_{t} \varphi\right\|_{H^{(1-\ell) / 2}\left(H^{\ell-1 / 2}\right)}^{2}\right. \\
& \left.+\left\|\theta^{*} A_{t} \varphi\right\|_{L^{2}\left(H^{1 / 2}\right)}^{2}\right) \leq C \mathrm{e}^{C T\|A\|_{P}^{2}\left(1+\|A\|_{P}^{4}\right)\left(1+\|A\|_{H^{(3-\ell) / 2}\left(H^{\nu_{2}}\right)}^{2}\right)} \\
& \times\left[( 1 + \| a _ { t } \| _ { L ^ { 2 } ( L ^ { r } ) } ^ { 2 } + \| b _ { t } \| _ { L ^ { 2 } ( L ^ { r } ) } ^ { 2 } ) \left(\left\|\theta_{t}^{*} \varphi_{t}\right\|_{L^{2}(Q)^{N}}^{2}+\left\|\theta^{*} \varphi\right\|_{L^{2}\left(H^{2}\right)}^{2}+\left\|\theta_{t}^{*} \varphi\right\|_{L^{2}\left(H^{1}\right)}^{2}\right.\right. \\
& \left.+\|u\|_{L^{2}\left(H^{1}\right)}^{2}\right)+\left(\|a\|_{\infty}^{2}+\|b\|_{\infty}^{2}\right)\|u\|_{L^{2}\left(H^{1}\right)}^{2}+\|A\|_{H^{(3-\ell) / 2}\left(H^{\nu_{2}}\right)}^{2}\left(\left\|\theta_{t}^{*} \varphi\right\|_{L^{2}\left(H^{1}\right)}^{2}\right. \\
& \left.\left.+\|u\|_{L^{2}\left(H^{1}\right)}^{2}+\left\|\theta^{*} \varphi\right\|_{L^{2}\left(H^{2}\right)}^{2}\right)\right] \text {. }
\end{aligned}
$$


Combining this with (92), we obtain

$$
\begin{aligned}
& \left\|\theta^{*} \varphi_{t t}\right\|_{L^{2}(Q)^{N}}^{2} \leq C \mathrm{e}^{C T\left(\|a\|_{\infty}^{2}+\|b\|_{\infty}^{2}+\|A\|_{P}^{2}\right)}\left(1+\|A\|_{P}^{4}\right) \\
& \times\left(1+\|A\|_{H^{(3-\ell) / 2}\left(H^{\left.\nu_{2}\right)}\right)}^{2}\right)\left(1+\|a\|_{\infty}^{2}+\|b\|_{\infty}^{2}+\left\|a_{t}\right\|_{L^{2}\left(L^{r}\right)}^{2}+\left\|b_{t}\right\|_{L^{2}\left(L^{r}\right)}^{2}\right. \\
& \left.+\|A\|_{H^{(3-\ell) / 2}\left(H^{\nu_{2}}\right)}^{2}\right)\left(\left\|\theta_{t}^{*} \varphi_{t}\right\|_{L^{2}(Q)^{N}}^{2}+\left\|\theta^{*} \varphi\right\|_{L^{2}\left(H^{2}\right)}^{2}+\left\|\theta_{t}^{*} \varphi\right\|_{L^{2}\left(H^{1}\right)}^{2}\right) .
\end{aligned}
$$

Taking now $\lambda \geq C \mathrm{e}^{C T\left(\|a\|_{\infty}^{2}+\|b\|_{\infty}^{2}+\|A\|_{\infty}^{2}\right)}\left(1+\|a\|_{\infty}^{10 / 3}+\|b\|_{L^{\infty}(Q)^{N}}^{10 / 3}+\left\|a_{t}\right\|_{L^{2}\left(L^{r}\right)}^{10 / 3}+\left\|b_{t}\right\|_{L^{2}\left(L^{r}\right)}^{10 / 3}+\|A\|_{P}^{5}+\|A\|_{H^{(3-\ell) / 2}\left(H^{\nu_{2}}\right)}^{10 / 3}\right)$, we have

$$
\begin{aligned}
& \left\|\theta^{*} \varphi_{t t}\right\|_{L^{2}(Q)^{N}}^{2} \leq C \lambda^{2}\left(\left\|\theta_{t}^{*} \varphi_{t}\right\|_{L^{2}(Q)^{N}}^{2}+\left\|\theta^{*} \varphi\right\|_{L^{2}\left(H^{2}\right)}^{2}+\left\|\theta_{t}^{*} \varphi\right\|_{L^{2}(W)}^{2}\right) \\
& \leq C \lambda^{2}\left(\lambda^{-2} T^{2} s^{-3 / 2} \mathrm{e}^{4 \lambda\left\|\eta^{0}\right\|_{\infty}} \iint_{Q} \mathrm{e}^{-2 s \alpha^{*}} \widehat{\xi}^{-1}\left|\varphi_{t}\right|^{2} \mathrm{~d} x \mathrm{~d} t\right.
\end{aligned}
$$

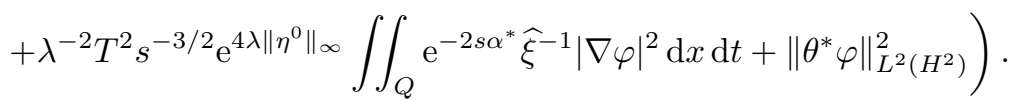

In order to estimate the last term, let us set $(\widehat{\varphi}, \widehat{\pi})=\theta^{*}(\varphi, \pi)$. They fulfill

$$
\begin{cases}-\widehat{\varphi}_{t}-\Delta \widehat{\varphi}-(a(x, t), \nabla) \widehat{\varphi}-D \widehat{\varphi} b(x, t)+\nabla \widehat{\pi}=-\theta_{t}^{*} \varphi & \text { in } Q, \\ \nabla \cdot \widehat{\varphi}=0 & \text { in } Q, \\ \widehat{\varphi} \cdot n=0,(\sigma(\widehat{\varphi}, \widehat{\pi}) \cdot n)_{t g}+(A(x, t) \widehat{\varphi})_{t g}=0 & \text { on } \Sigma, \\ \widehat{\varphi}(\cdot, T)=0 & \text { in } \Omega .\end{cases}
$$

Similarly as we did in (85), we find:

$$
\begin{aligned}
& \|\widehat{\varphi}\|_{H^{1}\left(L^{2}\right) \cap L^{2}\left(H^{2}\right)}^{2} \leq C \mathrm{e}^{C T\left(\|a\|_{\infty}^{2}+\|b\|_{\infty}^{2}+\|A\|_{P}^{2}\right)}\left(1+\|A\|_{P}^{4}\right) \times \\
& \quad \times\left(1+\|a\|_{\infty}^{2}+\|b\|_{\infty}^{2}\right)\left\|\theta_{t}^{*} \varphi\right\|_{L^{2}(Q)^{N}}^{2} \\
& \quad \leq C \mathrm{e}^{C T\left(\|a\|_{\infty}^{2}+\|b\|_{\infty}^{2}+\|A\|_{P}^{2}\right)}\left(1+\|A\|_{P}^{4}\right)\left(1+\|a\|_{\infty}^{2}+\|b\|_{\infty}^{2}\right) \times \\
& \quad \times s^{-3 / 2} \lambda^{-2} T^{2} \mathrm{e}^{4 \lambda\left\|\eta^{0}\right\|} \iint_{Q} \mathrm{e}^{-2 s \alpha^{*}}(\widehat{\xi})^{-1}|\varphi|^{2} \mathrm{~d} x \mathrm{~d} t .
\end{aligned}
$$

Taking $\lambda \geq C \mathrm{e}^{C T\left(\|a\|_{\infty}^{2}+\|b\|_{\infty}^{2}+\|A\|_{P}^{2}\right)}\left(1+\|a\|_{\infty}^{10 / 3}+\|b\|_{\infty}^{10 / 3}+\|A\|_{P}^{5}\right)$ and $s \geq C \mathrm{e}^{8 \lambda\left\|\eta^{0}\right\|_{\infty}}\left(T^{4}+T^{8}\right)$, we obtain from (93)

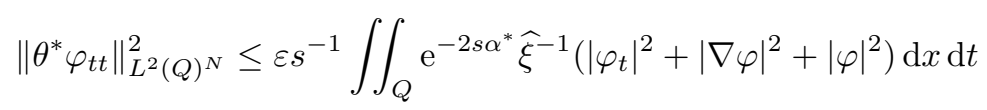

for a small positive constant $\varepsilon(\Omega, \omega)$.

Once $\theta^{*} \varphi_{t t}$ has been bounded, we come back to expressions (89) and (90) and we deduce

$$
\begin{aligned}
& s^{2} \lambda^{3} \iint_{\omega^{\prime} \times(0, T)} \mathrm{e}^{-2 s \widehat{\alpha} \widehat{\xi}^{2}}\left|\varphi_{t}\right|^{2} \mathrm{~d} x \mathrm{~d} t \leq C\left(s^{15 / 2} \lambda^{8} \iint_{\omega^{\prime} \times(0, T)} \mathrm{e}^{-4 s \widehat{\alpha}+2 s \alpha^{*}} \widehat{\xi}^{15 / 2}|\varphi|^{2} \mathrm{~d} x \mathrm{~d} t\right.
\end{aligned}
$$

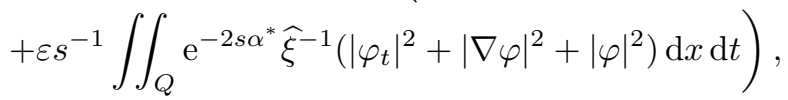

for

$$
\lambda \geq C \mathrm{e}^{C T\left(\|a\|_{\infty}^{2}+\|b\|_{\infty}^{2}+\|A\|_{P}^{2}\right)}\left(1+\|a\|_{\infty}^{10 / 3}+\|b\|_{\infty}^{10 / 3}+\left\|a_{t}\right\|_{L^{2}\left(L^{r}\right)}^{10 / 3}+\left\|b_{t}\right\|_{L^{2}\left(L^{r}\right)}^{10 / 3}+\|A\|_{P}^{5}+\|A\|_{H^{(3-\ell) / 2}\left(H^{\nu 2}\right)}^{10 / 3}\right)
$$

and $s \geq C \mathrm{e}^{8 \lambda\left\|\eta^{0}\right\|_{\infty}}\left(T^{4}+T^{8}\right)$. 
Therefore, by virtue of (88), we find (see (48) for the expression of $I(s, \lambda ; \varphi)$ )

$$
\begin{aligned}
& I(s, \lambda ; \varphi) \leq C(1+T)\left(s^{15 / 2} \lambda^{8} \iint_{\omega^{\prime} \times(0, T)} \mathrm{e}^{-4 s \widehat{\alpha}+2 s \alpha^{*}} \widehat{\xi}^{15 / 2}|\varphi|^{2} \mathrm{~d} x \mathrm{~d} t\right.
\end{aligned}
$$

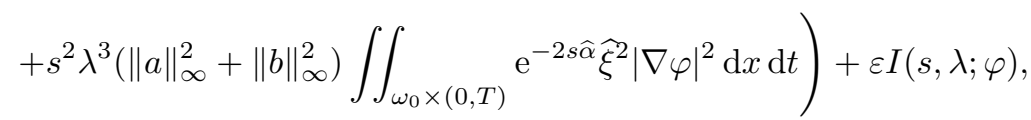

for

$$
\lambda \geq C \mathrm{e}^{C T\left(\|a\|_{\infty}^{2}+\|b\|_{\infty}^{2}+\|A\|_{P}^{2}\right)}\left(1+\|a\|_{\infty}^{10 / 3}+\|b\|_{\infty}^{10 / 3}+\left\|a_{t}\right\|_{L^{2}\left(L^{r}\right)}^{10 / 3}+\left\|b_{t}\right\|_{L^{2}\left(L^{r}\right)}^{10 / 3}+\|A\|_{P}^{5}+\|A\|_{H^{(3-\ell) / 2}\left(H^{\nu_{2}}\right)}^{10 / 3}\right)
$$

and $s \geq C \mathrm{e}^{8 \lambda\left\|\eta^{0}\right\|_{\infty}}\left(T^{4}+T^{8}\right)$.

Finally, the last term can be eliminated, first using a cut-off function $\rho \in C^{2}(\bar{\omega})$ with

$$
\rho \equiv 1 \text { in } \omega_{0}, \operatorname{supp} \rho \subset \subset \omega
$$

and then the term

$$
s^{-1} \iint_{Q} \mathrm{e}^{-2 s \alpha} \xi^{-1}|\Delta \varphi|^{2} \mathrm{~d} x \mathrm{~d} t
$$

appearing in the expression of $I(s, \lambda ; \varphi)$ (as we did in (77)). Indeed, we have

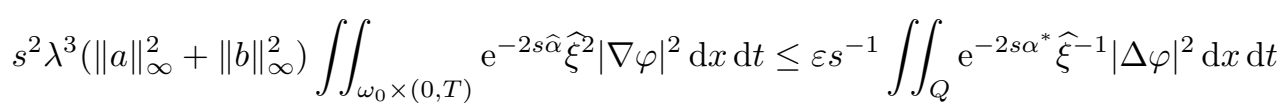

$$
\begin{aligned}
& +C s^{5} \lambda^{6}\left(\|a\|_{\infty}^{4}+\|b\|_{\infty}^{4}\right) \iint_{\omega \times(0, T)} \mathrm{e}^{-4 s \widehat{\alpha}+2 s \alpha^{*}} \widehat{\xi}^{5}|\varphi|^{2} \mathrm{~d} x \mathrm{~d} t
\end{aligned}
$$

for a small positive constant $\varepsilon(\Omega, \omega)$. From this and (94), it easily follows inequality (80).

This ends the proof of Proposition 2.3.

\section{Local NUll CONTROllability of the NAVIER-Stokes SySTEM}

In this section, Theorems 0.1 and 0.2 will be proved. In a first paragraph, we will provide the proof of the null controllability result for system (2), while the local exact controllability to the trajectories of (1) will be given in the last paragraph.

\subsection{Proof of theorem 0.1}

Proof. Let us remark that the proof of this result follows the ideas of [3]. Let us first consider, for each $\varepsilon>0$, the extremal problem

$$
\inf _{(w, v) \text { solve (2) }} \frac{1}{2} \iint_{\omega \times(0, T)} \rho(t)|v|^{2} \mathrm{~d} x \mathrm{~d} t+\frac{1}{2 \varepsilon}\|w(T)\|_{L^{2}(\Omega)^{N}}^{2} .
$$

Here, we have denoted

$$
\rho(t)=\mathrm{e}^{4 s \widehat{\alpha}-2 s \alpha^{*}} \widehat{\xi}^{-15 / 2},
$$

where $s$ and $\lambda$ are chosen like in Proposition 2.3 and the definition of $\widehat{\alpha}, \alpha^{*}$ and $\widehat{\xi}$ was given in (44). Furthermore, $w$ is the solution of (2) associated to $v$. 
Let us suppose that there exists a (unique) solution of $(95)\left(w_{\varepsilon}, v_{\varepsilon}\right)$ with $\rho(t)^{1 / 2} v_{\varepsilon}$ belonging to $L^{2}(\omega \times(0, T))^{N}$. The necessary condition of minimum yields

$$
\iint_{\omega \times(0, T)} \rho(t) v_{\varepsilon} \cdot v \mathrm{~d} x \mathrm{~d} t+\frac{1}{\varepsilon} \int_{\Omega} w_{\varepsilon}(T) \cdot u(T) \mathrm{d} x=0 \quad \forall v \in L^{2}(\omega \times(0, T))^{N},
$$

where $u$ is, together with certain pressure $\theta$, solution of

$$
\left\{\begin{array}{lc}
u_{t}-\nabla \cdot(D u)+(a(x, t), \nabla) u+(b(x, t), \nabla) u & +(u, \nabla) b(x, t)+\nabla \theta=v 1_{\omega} \\
\nabla \cdot u=0 & \text { in } Q, \\
u \cdot n=0,(\sigma(u, \theta) \cdot n)_{t g}+(A(x, t) u)_{t g}=0 & \text { in } Q, \\
u(\cdot, 0)=0 & \text { in } \Sigma,
\end{array}\right.
$$

Let us now introduce $\left(\varphi_{\varepsilon}, \pi_{\varepsilon}\right)$ solution of the following homogeneous adjoint system:

$$
\begin{cases}-\varphi_{\varepsilon, t}-\nabla \cdot\left(D \varphi_{\varepsilon}\right)-(a(x, t), \nabla) \varphi_{\varepsilon}-D \varphi_{\varepsilon} b(x, t)+\nabla \pi_{\varepsilon}=0 & \text { in } Q, \\ \nabla \cdot \varphi_{\varepsilon}=0 & \text { in } Q, \\ \varphi_{\varepsilon} \cdot n=0,\left(\sigma\left(\varphi_{\varepsilon}, \pi_{\varepsilon}\right) \cdot n\right)_{t g}+\left(A^{t}(x, t) \varphi_{\varepsilon}\right)_{t g}=0 & \text { on } \Sigma, \\ \varphi_{\varepsilon}(\cdot, T)=-\frac{1}{\varepsilon} w_{\varepsilon}(\cdot, T) & \text { in } \Omega .\end{cases}
$$

Then, the duality properties between $\varphi_{\varepsilon}$ and $w$ provides

$$
-\frac{1}{\varepsilon} \int_{\Omega} w_{\varepsilon}(T) \cdot u(T) \mathrm{d} x=\iint_{\omega \times(0, T)} v \cdot \varphi_{\varepsilon} \mathrm{d} x \mathrm{~d} t,
$$

which combined with (96), yields

$$
\iint_{\omega \times(0, T)} v \cdot \varphi_{\varepsilon} \mathrm{d} x \mathrm{~d} t=\iint_{\omega \times(0, T)} v \cdot\left(\rho(t) v_{\varepsilon}\right) \mathrm{d} x \mathrm{~d} t \quad \forall v \in L^{2}(\omega \times(0, T))^{N} .
$$

Consequently, we can identify $v_{\varepsilon}$ :

$$
v_{\varepsilon}=\rho(t)^{-1} \varphi_{\varepsilon} 1_{\omega}
$$

From the systems fulfilled by $w_{\varepsilon}$ and $\varphi_{\varepsilon}$, we find

$$
-\frac{1}{\varepsilon}\left\|w_{\varepsilon}(T)\right\|_{L^{2}(\Omega)^{N}}^{2}=\iint_{\omega \times(0, T)} \rho(t)^{-1}\left|\varphi_{\varepsilon}\right|^{2} \mathrm{~d} x \mathrm{~d} t+\int_{\Omega} \varphi_{\varepsilon}(0) \cdot w^{0} \mathrm{~d} x .
$$

The Carleman inequality (80) used for $\varphi_{\varepsilon}$ tells us that

$$
\left\|\varphi_{\varepsilon}(0)\right\|_{L^{2}(\Omega)^{N}}^{2} \leq C(\Omega, \omega, T, a, b, A) \iint_{\omega \times(0, T)} \rho(t)^{-1}\left|\varphi_{\varepsilon}\right|^{2} \mathrm{~d} x \mathrm{~d} t,
$$

so

$$
\frac{1}{\varepsilon}\left\|w_{\varepsilon}(T)\right\|_{L^{2}(\Omega)^{N}}^{2}+\left\|\rho(t)^{1 / 2} v_{\varepsilon}\right\|_{L^{2}(\omega \times(0, T))^{N}}^{2} \leq C\left\|w^{0}\right\|_{H}^{2} \quad \forall \varepsilon>0 .
$$

Consequently, we deduce the existence of a control $v$ such that $\rho(t)^{1 / 2} v \in L^{2}(\omega \times(0, T))^{N}$ (whose corresponding solution we denote by $w$ ) such that (3) holds and

$$
\left\|\rho(t)^{1 / 2} v\right\|_{L^{2}(\omega \times(0, T))^{N}} \leq C(\Omega, \omega, T, a, b, A)\left\|w^{0}\right\|_{H} .
$$


Let us finally bound the $H^{1}\left(L^{2}\right)$ and the $L^{\infty}\left(H^{1}\right)$ norms of this control. For this, let us introduce the functions $\left(\psi_{\varepsilon}, q_{\varepsilon}\right)=\rho(t)^{-1}\left(\varphi_{\varepsilon}, \pi_{\varepsilon}\right)$. They verify:

$$
\begin{cases}-\psi_{\varepsilon, t}-\nabla \cdot\left(D \psi_{\varepsilon}\right)-(a, \nabla) \psi_{\varepsilon}-D \psi_{\varepsilon} b+\nabla q_{\varepsilon}=-\left(\rho(t)^{-1}\right)_{t} \varphi_{\varepsilon} & \text { in } Q \\ \nabla \cdot \psi_{\varepsilon}=0 & \text { in } Q \\ \psi_{\varepsilon} \cdot n=0,\left(\sigma\left(\psi_{\varepsilon}, q_{\varepsilon}\right) \cdot n\right)_{t g}+\left(A^{t}(x, t) \psi_{\varepsilon}\right)_{t g}=0 & \text { on } \Sigma \\ \psi_{\varepsilon}(\cdot, T)=0 & \text { in } \Omega .\end{cases}
$$

Applying here the a priori estimate obtained in Proposition 1.1, we have

$$
\left\|\psi_{\varepsilon, t}\right\|_{L^{2}(Q)^{N}}+\left\|\psi_{\varepsilon}\right\|_{L^{\infty}\left(H^{1}\right)} \leq C(\Omega, \omega, a, b, A)\left\|\left(\rho(t)^{-1}\right)_{t} \varphi_{\varepsilon}\right\|_{L^{2}(Q)^{N}},
$$

which combined with (80), implies

$$
\left\|v_{\varepsilon, t}\right\|_{L^{2}(\omega \times(0, T))^{N}}+\left\|v_{\varepsilon}\right\|_{L^{\infty}\left(H^{1}\right)} \leq C(\Omega, \omega, T, a, b, A)\left\|\rho(t)^{1 / 2} v_{\varepsilon}\right\|_{L^{2}(\omega \times(0, T))^{N}} .
$$

From (98), we conclude that $v \in H^{1}\left(0, T ; L^{2}(\omega)^{N}\right)$ and

$$
\|v\|_{H^{1}\left(L^{2}\right)}+\|v\|_{L^{\infty}\left(H^{1}\right)} \leq C(\Omega, \omega, T, a, b, A)\left\|y^{0}\right\|_{H} .
$$

Remark 3.1. The control previously found can be constructed in such a way that it acts over the system (2) in the form $\zeta v$ instead of $v 1_{\omega}$, where $\zeta$ is a cut-off function with support in $\omega$. The proof of this would be exactly the same of Proposition 0.1 except for the corresponding change in system (97).

\subsection{Proof of theorem 0.2}

Proof. Let us subtract system (4) fulfilled by $\bar{y}$ from (1). Denoting $w=y-\bar{y}$, we have:

$$
\begin{cases}w_{t}-\Delta w+(w, \nabla) w+(w, \nabla) \bar{y}+(\bar{y}, \nabla) w+\nabla q=v 1_{\omega} & \text { in } Q, \\ \nabla \cdot w=0 & \text { in } Q, \\ w \cdot n=0,(\sigma(w, q) \cdot n)_{t g}+(F(\bar{y} ; w) w)_{t g}=0, & \text { on } \Sigma, \\ w(0)=y^{0}-\bar{y}^{0}=w^{0} & \text { in } \Omega,\end{cases}
$$

where

$$
F(\bar{y} ; w)=\int_{0}^{1} \nabla f(\bar{y}+l w) \mathrm{d} l \in \mathbf{R}^{N} \times \mathbf{R}^{N} .
$$

With this notation, our goal is to find a control $v$ such that $w$ solution to (99) satisfies (3). This would end the proof of Theorem 0.2 .

Let us introduce the Banach space

$$
Z=\left\{z \in H^{(3-\ell) / 2}\left(0, T ; H^{\nu_{2}+1 / 2}(\Omega)^{N} \cap W\right) \cap H^{1-\ell}\left(0, T ; W^{\nu_{1}+1 / 2, \nu_{1}+1}(\Omega)^{N} \cap W\right)\right\}
$$

$\left(\ell, \nu_{1}\right.$ and $\nu_{2}$ were defined in the introduction, at the beginning of the paper) and the closed linear manifold

$$
Z_{0}=\left\{z \in Z: z(\cdot, 0)=w^{0} \text { in } \Omega\right\} .
$$


Then, for each $z \in Z_{0}$ and by virtue of Theorem 0.1 and Remark 3.1, there exists a control $v_{z} \in H^{1}\left(0, T ; L^{2}(\omega)^{N}\right) \cap$ $C^{0}\left([0, T] ; H^{1}(\omega)^{N}\right)$ such that the solution $w_{z}$ of

$$
\begin{cases}w_{t}-\nabla \cdot(D w)+(z, \nabla) w+(w, \nabla) \bar{y}+(\bar{y}, \nabla) w+\nabla p=\zeta v_{z} & \text { in } Q \\ \nabla \cdot w=0 & \text { in } Q \\ w \cdot n=0,(D w \cdot n)_{t g}+(F(\bar{y} ; z) w)_{t g}=0 & \text { on } \Sigma \\ w(\cdot, 0)=w^{0}(\cdot) & \text { in } \Omega\end{cases}
$$

verifies (3). Observe that, since $F \in C^{2}\left(\mathbf{R}^{N} ; \mathbf{R}^{N \times N}\right)$ (see (14)) and $\bar{y}$ verifies (12), $F(\bar{y}, z) \in Z$ for every $z \in Z_{0}$. Moreover, $v_{z}$ can be built such that

$$
\left\|v_{z}\right\|_{H^{1}\left(L^{2}\right)}+\left\|v_{z}\right\|_{L^{\infty}\left(L^{2}\right)} \leq C\left(\Omega, \omega, T,\|z\|_{Z},\|F(\bar{y} ; z)\|_{Z}\right)\left\|w^{0}\right\|_{H} .
$$

Next, since the terms

$$
(z, \nabla) w,(w, \nabla) \bar{y},(\bar{y}, \nabla) w, \zeta v_{z}
$$

belong to

$$
L^{\infty}\left(0, T ; H^{1}(\Omega)^{N}\right) \cap H^{1}\left(0, T ; L^{2}(\Omega)^{N}\right),
$$

they have null normal traces and $w^{0}=y^{0}-\bar{y}^{0}$ satisfies the compatibility condition

$$
\left(D w^{0} \cdot n\right)_{t g}+\left(F(\bar{y} ; z)(x, 0) w^{0}\right)_{t g}=0
$$

(from (13) and (15)), we can apply Proposition 1.2 and we obtain

$$
w_{z} \in H^{2}(0, T ; H) \cap H^{1}\left(0, T ; H^{2}(\Omega)^{N} \cap W\right)
$$

for each $z \in Z_{0}$.

Let us introduce the space

$$
\tilde{Z}=H^{1}\left(0, T ; H^{2}(\Omega) \cap W\right) \cap H^{2}(0, T ; H) .
$$

Observe that $\tilde{Z}$ is compactly embedded into $Z$. Indeed, this can be deduced from $H^{2}(\Omega) \subset W^{\nu_{1}+1 / 2, \nu_{1}+1}(\Omega)$ compactly (recall that $\nu_{1}>1$ arbitrarily small) and

$$
\tilde{Z} \subset H^{(3-(\ell-\varepsilon)) / 2}\left(0, T ; H^{\nu_{2}+1 / 2+\varepsilon}(\Omega)^{N}\right) \cap H^{1}\left(0, T ; H^{2}(\Omega)^{N}\right) \quad \text { continuously }
$$

for positive $\varepsilon$ arbitrarily small (in fact, we can even take $\varepsilon<1 / 2$ ). This can be established by interpolation spaces arguments.

Let $\Lambda(z)$ be the set constituted by the controls $v_{z} \in H^{1}\left(0, T ; L^{2}(\omega)^{N}\right) \cap C^{0}\left([0, T] ; H^{1}(\omega)^{N}\right)$ driving the solution $w_{z}$ of system (100) to zero at time $T$ and such that (101) holds. On the other hand, let us introduce their associated states

$$
A(z)=\left\{w_{z} \text { solution of }(100): v_{z} \in \Lambda(z)\right\} .
$$

Observe that $A(z) \subset \tilde{Z} \stackrel{c}{\subset} Z$. Furthermore, for every $z \in Z$,

$$
\left\|w_{z}\right\|_{Z} \leq \tilde{C}\left(\Omega, \omega, T,\|z\|_{Z},\|F(\bar{y} ; z)\|_{Z}\right)\left\|w^{0}\right\|_{H^{3} \cap W},
$$

for certain positive constant $\tilde{C}$.

Our goal is to prove that the set-valued mapping

$$
z \longmapsto A(z)
$$


has a fixed point with the additional hypothesis $\left\|w^{0}\right\|_{H^{3} \cap W} \leq \delta(\Omega, \omega, T, \ell, \nu)$. This would finish the proof of Theorem 0.2. To this end, we will apply Kakutani's theorem (see [2]): let

$$
A: Z_{0} \longmapsto Z_{0}
$$

be a set-valued mapping such that

- $A(z)$ is a nonempty closed convex set of $Z_{0}$, for every $z \in Z_{0}$.

- There exists a convex compact set $K \subset Z_{0}$ such that $A(K) \subset K$.

- $A$ is upper-hemicontinuous in $Z_{0}$, i.e., $\forall \lambda \in Z_{0}^{\prime}$, the mapping

$$
z \longmapsto \sup _{w \in A(z)}\langle\lambda, w\rangle_{Z_{0}^{\prime} Z_{0}}
$$

is upper semicontinuous.

Then, there exists $z \in K$ such that $z \in A(z)$.

The first item is readily satisfied. In order to prove the second one, let $M>0$ be given and let us denote

$$
C(M)=\sup _{\|z\|_{Z} \leq M} \tilde{C}\left(\Omega, \omega, T, \ell, \nu,\|z\|_{Z},\|F(\bar{y} ; z)\|_{Z}\right)
$$

where $\tilde{C}$ was introduced in (103). Then, with a choice like $\delta=M / \tilde{C}(M)$, we have that $A$ sends the closed convex set

$$
\tilde{K}=\left\{z \in Z_{0}:\|z\|_{Z} \leq M\right\}
$$

in a compact set $K \subset \tilde{K}$. This comes from the fact that $\tilde{Z}$ is compactly embedded into $Z$.

Let us finally prove the upper-hemicontinuity of $A$. Let

$$
z_{k} \rightarrow z \text { in } Z
$$

From the compactness of $A\left(z_{k}\right)$, we have that

$$
\sup _{w \in A\left(z_{k}\right)}\langle\lambda, w\rangle_{Z^{\prime} Z}=\left\langle\lambda, w_{k}\right\rangle_{Z^{\prime} Z}
$$

for certain $w_{k} \in A\left(z_{k}\right)$. We choose $\left\{z_{k^{\prime}}\right\} \subset\left\{z_{k}\right\}$ such that

$$
\limsup _{k \rightarrow \infty} \sup _{w \in A\left(z_{k}\right)}\langle\lambda, w\rangle_{Z^{\prime} Z}=\lim _{k^{\prime} \rightarrow \infty}\left\langle\lambda, w_{k^{\prime}}\right\rangle_{Z^{\prime} Z}
$$

and denote by $v_{k^{\prime}}$ the controls belonging to $\Lambda\left(z_{k^{\prime}}\right)$ associated to $w_{k^{\prime}}$, so that they fulfill the system

$$
\begin{cases}\partial_{t} w_{k^{\prime}}-\nabla \cdot\left(D w_{k^{\prime}}\right)+\left(z_{k^{\prime}}, \nabla\right) w_{k^{\prime}}+\left(z_{k^{\prime}}, \nabla\right) \bar{y}+(\bar{y}, \nabla) z_{k^{\prime}}+\nabla p_{k^{\prime}}=v_{k^{\prime}} \zeta & \text { in } Q \\ \nabla \cdot w_{k^{\prime}}=0 & \text { in } Q \\ w_{k^{\prime}} \cdot n=0,\left(D w_{k^{\prime}} \cdot n\right)_{t g}+\left(F\left(\bar{y} ; z_{k^{\prime}}\right) w_{k^{\prime}}\right)_{t g}=0 & \text { on } \Sigma \\ w_{k^{\prime}}(\cdot, 0)=w^{0}(\cdot) & \text { in } \Omega\end{cases}
$$

Then, using $F\left(z_{k^{\prime}}\right) \rightarrow F(z)$ in $Z$, estimates (103) and (101) and $\tilde{Z} \stackrel{c}{\subset} Z$, we find (at least for a subsequence)

$$
w_{k^{\prime}} \rightarrow w^{*} \text { in } Z
$$

and

$$
v_{k^{\prime}} \rightarrow v^{*} \text { weakly in } H^{1}\left(0, T ; L^{2}(\omega)^{N}\right) \cap L^{\infty}\left(0, T ; L^{2}(\omega)^{N}\right)
$$


It is not difficult then to deduce that $v^{*} \in \Lambda(z)$ and $w^{*} \in A(z)$. Hence,

$$
\lim _{k^{\prime} \rightarrow \infty} \sup _{w \in A\left(z_{k^{\prime}}\right)}\langle\lambda, w\rangle_{Z^{\prime} Z}=\left\langle\lambda, w^{*}\right\rangle_{Z^{\prime} Z} \leq \sup _{w \in A(z)}\langle\lambda, w\rangle_{Z^{\prime} Z}
$$

as we wanted to prove.

Acknowledgements. The author is grateful to Prof. E. Fernández-Cara for his numerous suggestions and fruitful discussions, which has definitively improved this paper.

\section{REFERENCES}

[1] R.A. Adams, Sobolev spaces. Pure and Applied Mathematics, Vol. 65. Academic Press, New York-London, 1975.

[2] J.-P. Aubin, L'analyse non linéaire et ses motivations économiques. Masson, Paris (1984).

[3] S. Anita and V. Barbu, Null controllability of nonlinear convective heat equations. ESAIM: COCV 5 (2000) $157-173$.

[4] J.A. Bello, Thesis, University of Seville (1993).

[5] T. Cebeci and A.M. Smith, Analysis of turbulent boundary layers. Applied Mathematics and Mechanics, No. 15. Academic Press [Harcourt Brace Jovanovich, Publishers], New York-London (1974).

[6] J.-M. Coron, On the controllability of the 2-D incompressible Navier-Stokes equations with the Navier slip boundary conditions. ESAIM: COCV $1(1995 / 96) 35-75$.

[7] C. Fabre, J.-P. Puel and E. Zuazua, Approximate controllability of the semilinear heat equation. Proc. Roy. Soc. Edinburgh 125A (1995) 31-61.

[8] E. Fernández-Cara, S. Guerrero, O.Yu. Imanuvilov and J.-P. Puel, Local exact controllability of the Navier-Stokes system. J. Math. Pures Appl. 83/12 (2004) 1501-1542.

[9] E. Fernández-Cara and E. Zuazua, Null and approximate controllability for weakly blowing up semilinear heat equations. Ann. Inst. H. Poincaré, Analyse non Lin. 17 (2000) 583-616.

[10] A. Fursikov and O.Yu. Imanuvilov, Controllability of Evolution Equations. Lecture Notes \#34, Seoul National University, Korea (1996).

[11] G.P. Galdi, An introduction to the Mathematical Theory of the Navier-Stokes equations, Vol. I. Springer-Verlag, New York (1994).

[12] O.Yu. Imanuvilov, Local exact controllability for the 2-D Navier-Stokes equations with the Navier slip boundary conditions, in Turbulence Modelling and Vortex Dynamics, Istanbul, Springuer Berlin, 1996. Lect. Notes . Phys. 491 (1997) 148-168

[13] O.Yu. Imanuvilov, Remarks on exact controllability for the Navier-Stokes equations. ESAIM: COCV 6 (2001) 39-72.

[14] O.Yu. Imanuvilov and J.-P. Puel, Global Carleman estimates for weak elliptic non homogeneous Dirichlet problem. Int. Math. Research Notices 16 (2003) 883-913.

[15] O.Yu. Imanuvilov and M. Yamamoto, Carleman estimate for a parabolic equation in a Sobolev space of negative order and its applications. Lect. Notes Pure Appl. Math. 218 (2001)

[16] J.-L. Lions and E. Magenes, Problèmes aux limites non homogènes et applications (3 volumes). Dunod, Gauthiers-Villars, Paris (1968).

[17] P. Malliavin, Intégration et probabilités. Analyse de Fourier et analyse spectrale. Masson (1982).

[18] R.L. Panton, Incompressible flow. Wiley-Interscience, New York (1984).

[19] H. Schlichting, Boundary-Layer Theory. McGraw-Hill, New York (1968).

[20] V.A. Solonnikov and V.E. Schadilov, On a boundary value problem for a stationnary system of Navier-Stokes equations. Trudy Mat. Inst. Steklov 125 (1973) 196-210.

[21] L. Tartar, An introduction to Sobolev spaces and interpolation spaces. Course (2000), URL: http://www . math. cmu.edu/cna/publications/SOB+Int.pdf .

[22] R. Temam, Navier-Stokes equations. Theory and numerical analysis. Studies in Mathematics and its applications, 2. North Holland Publishing Co., Amsterdam-New York-Oxford (1977).

[23] E. Zuazua, Exact boundary controllability for the semilinear wave equation, H. Brezis and J.L. Lions Eds., Pitman, New York in Nonlinear Partial Differential Equations Appl. X (1991) 357-391. 